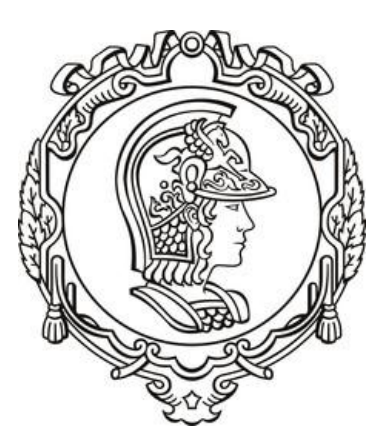

POLYTECHNIC SCHOOL OF THE UNIVERSITY OF SÃO PAULO Chemical Engineering Program

\author{
LILIANA PATRICIA OLIVO ARIAS
}

MODELING AND EXPERIMENTAL STUDY OF INVERSE SUSPENSION POLYMERIZATION OF ACRYLIC ACID AND TRIMETHYLOLPROPANE TRIACRYLATE FOR HYDROGEL PRODUCTION 


\section{MODELING AND EXPERIMENTAL STUDY OF INVERSE SUSPENSION POLYMERIZATION OF ACRYLIC ACID AND TRIMETHYLOLPROPANE TRIACRYLATE FOR HYDROGEL PRODUCTION}

Dissertation presented to the Chemical Engineering program of University of São Paulo as part of the requisites to obtain the tittle of M.Sc. in Chemical Engineering

Concentration area Chemical Engineering

Supervisor Prof. Dr Reinaldo Giudici 


\section{LILIANA PATRICIA OLIVO ARIAS}

\section{IMPROVING OF ENGINEERING COURSE}

Dissertation presented to the Chemical Engineering program of University of São Paulo as part of the requisites to obtain the tittle of M.Sc. in Chemical Engineering

Concentration area System process Engineering

Supervisor Prof. Dr Reinaldo Giudici

\section{V.1}

SÃO PAULO

2016 
Este exemplar foi revisado e corrigido em relação à versão original, sob responsabilidade única do autor e com a anuência de seu orientador.

São Paulo, de de

Assinatura do autor:

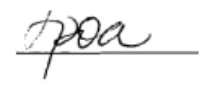

Assinatura do orientador:

Catalogação-na-publicação

Olivo Arias, Liliana

MODELING AND EXPERIMENTAL STUDY OF INVERSE

SUSPENSION POLYMERIZATION OF ACRYLIC ACID AND

TRIMETHYLOLPROPANE TRIACRYLATE FOR HYDROGEL PRODUCTION

/ L. Olivo Arias, R. Giudici -- versão corr. -- São Paulo, 2016.

$144 \mathrm{p}$.

Dissertação (Mestrado) - Escola Politécnica da Universidade de São Paulo. Departamento de Engenharia Química.

1.Mathematical Modeling 2.Kinetic Modeling 3.Inverse Suspension Polymerization 4.Cross-linking/Hydrogels 5.Numerical Fractionation Technique I.Universidade de São Paulo. Escola Politécnica. Departamento de Engenharia Química II.t. III.Giudici, Reinaldo 
"I dedicate my dissertation

To GOD, my PARENTS whose efforts helped me to accomplish my goal and learn how I could move in this crazy world" 


\section{ACKNOWLEDGEMENTS}

I would like to thank my advisor, Prof. Dr. Reinaldo Giudici for his much appreciated orientation, motivation and patience, as well as for giving me the opportunity to work with him and improve my knowledge.

To my Research Group: Paula Ambrogi, Maria Magdalena Espinola, Esmar Souza, Vinicius Nobre, Natalia Barbosa, Giuliana Torraga, Camila Emilia, Veronica Carranza, Victor Postal, Bruna Mattos and Dennis Chicoma, for their wise advice and support during my stay in Brazil.

To Leandro Aguiar, Paulo Moreira, Rodrigo Ramos, André Yamashita and Joel Mendes for supplying their lab expertise, materials, equipment and computational knowledge.

To Lidiane Andrade, Marco Antonio Stefano, Ana Fadeu, Maria Anita Mendes and engineering technicians for analyzing my samples and supplying material and their expertise.

To the University of São Paulo, specially to the research personnel that worked with me at the Semi-industrial building, secretaries, teachers, library personnel and colleagues who helped me in my experimental studies.

To my Church Group, Roommates and Friends here in São Paulo, Oscar, Any, Hector, Mario, Rose, Paulo and Maria Clara, for supporting me in my difficult moments and helping me in my academic studies

To CAPES Foundation, for supporting my stance in São Paulo, for 2 years and FDTE Foundation for helping me to finish my work. 
"The first and best victory is to conquer self." - Plato 


\begin{abstract}
In the present work, a super water-absorbent poly(acrylic acid) was synthetized by inverse suspension polymerization, using Span60 as the dispersant, toluene as the dispersing organic phase, trimethylolpropane triacrylate as the crosslinking agent, and sodium persulfate as the initiator. The synthesis was conducted in a smallscale glass reactor operated in semi-batch mode. The following reaction conditions were evaluated: effects of initiator concentration, temperature, percentage of multifunctional cross-linker agent and monomer concentration. Also, two important properties were determined, conversion and gel fraction. A kinetic model including a population balance was employed to simulate the process. The proposed model uses the numerical fractionation technique and is capable of predicting the pre-gel and post-gel properties, the effect of the crosslinking agent level on the polymer properties and the dynamic of gelation. The model was compared with the experimental results and showed a satisfactory representation of the system after parameter adjusting.
\end{abstract}

Keywords: Mathematical and Kinetic Modeling; Inverse Suspension Polymerization; Superabsorbent (poly-acrylic acid); Cross-linking, Numerical Fractionation Technique. 


\section{FIGURES LIST}

Figure 1 - Scheme of propagation and crosslinking reactions in FCC of vinyl/divinyl monomers beyond the gel point. $\mathrm{kp}_{\mathrm{i}}(\mathrm{i}=1$, and 2$)$ is the instantaneous propagation

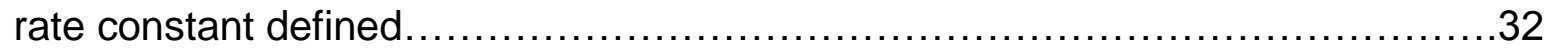
Figure 2- Predicted dynamics of the weight fraction of $\mathrm{gel}(\mathrm{wg})$ and monomer

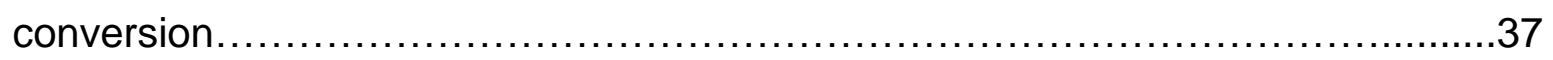

Figure 3 - Predicted dynamics of the weigth fraction of gel (wg). Effect of the initial

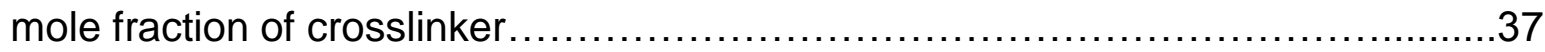

Figure 4- Predicted time evolution of monomer conversion and $\mathrm{Wg} \ldots \ldots \ldots \ldots \ldots . \ldots 38$ Figure 5- Predicted dynamics of the weigth fraction of gel with different crosllinker

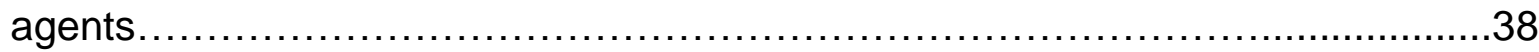

Figure 6- Inverse suspension polymerization in a batch reactor..................53

Figure 7 - Numerical fractionational method - transition of generation................61

Figure 8- Schematic of the reactor used in the inverse suspension polymerization

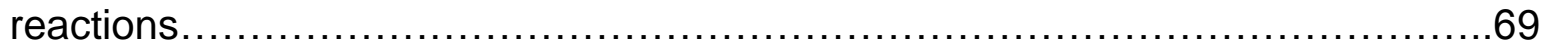

Figure 9- Vacuum Distillation equipment used for removing the MEHQ inhibitor.

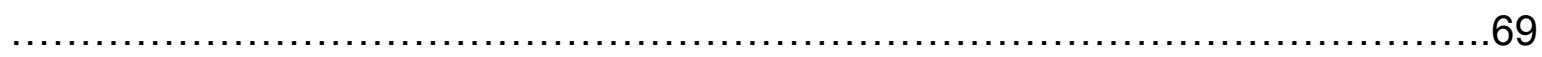

Figure 10- Samples for determination by gravimetry method........................73

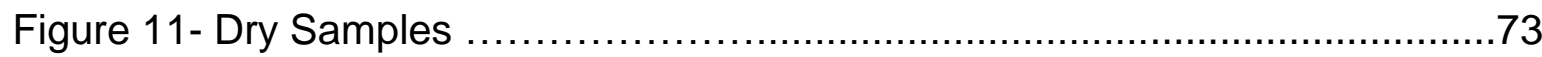

Figure 12- Soxhlet extraction equipment........................................ 74

Figure 13- Conversion-time homopolymerization of acrylic acid in run E12 .77

Figure 14- Conversion-time homopolymerization of acrylic acid during run E13 and

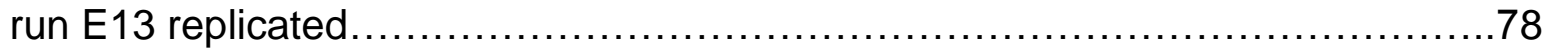

Figure 15- Conversion-time inverse suspension polymerization at different temperatures. 
Figure 16- Conversion-time inverse suspension polymerization with TMPTA concentration Run E 10 ......

Figure 17- Conversion-time inverse suspension polymerization with different crosslinkers TAO and EGDMA Run E 15 and E 14.

Figure 18- Conversion-time inverse suspension polymerization at different Sodium Persulfate concentration Run E 2 and E 6 . 82

Figure 19- Conversion-time inverse suspension polymerization at different feed flow rate time Run E 2 and $\mathrm{E} 8$ .83

Figure 20- Conversion-Polymerization rate of inverse suspension polymerization Run E8. 84

Figure 21- Conversion-Polymerization rate of inverse suspension polymerization Run E2 84

Figure 22- Conversion-time inverse suspension different acrylic acid concentration Run E2 and E9. .85

Figure 23- Polymerization rate and inhibition time as a function of deoxygenation conditions.No deoxygenation. 87

Figure 24- Polymerization rate and inhibition time as a function of deoxygenation conditions. 15 min deoxygenation .88

Figure 25- Polymerization rate and inhibition time as a function of deoxygenation conditions. 30 min deoxygenation .88

Figure 26- Polymerization rate and inhibition time as a function of deoxygenation conditions. 60 min deoxygenation 
Figure 27- Weight Fraction of Gel at different Crosslinkers concentration TMPTA 0.0205 . 90

Figure 28- Weight Fraction of Gel at different Crosslinkers concentration TAO and EGDMA 0.0205\% .91

Figure 29- Comparison between experimentally observed and predicted dynamics of monomer conversion and weight fraction of gel during the copolymerization of acrylic acid and two different crosslinker with tetraallyloxyethane and Etheylene glycol dimetricrylate.

Figure 30- Conversion-time Homopolymerization process at $60^{\circ} \mathrm{C}$..... .95

Figure 31-Conversion-time dependence of the rate coefficient for the homopropagation of acrylic acid $\mathrm{Kp}_{11} 70^{\circ} \mathrm{C}$ .96

Figure 32- Conversion-time dependence rate coefficient for the homopropagation of acrylic acid $\mathrm{Kp}_{11} 60^{\circ} \mathrm{C}$ .97

Figure 33- Conversion-Time different values of the rate coefficient for the homopropagation of acrylic acid $\mathrm{Kp}_{11} 50^{\circ} \mathrm{C}$

Figure 34- Conversion-time different values of rate coefficient homopropagation of acrylic acid (Kp11) influence of crosslinkers TAO. 98

Figure 35- Conversion-time dependence of the rate coefficient homopropagation of acrylic acid (Kp11) influence of crosslinkers TMPTA $60^{\circ} \mathrm{C}$

Figure 36- Conversion-time rate different values of the coefficient homopropagation of acrylic acid (Kp11) Influence of crosslinkers EGDMA. 99 
Figure 37- Conversion-time different values of the rate coefficient for the homopropagation of acrylic acid $\left(\mathrm{Kp}_{11}\right)$ influence low initiator concentration of sodium persulfate. 100

Figure 38- Conversion-time different values of the rate coefficient homopropagation of acrylic acid (Kp11)-influence on feed flow rate time 101

Figure 39- Conversion-time different values of the rate coefficient for the homopropagation of acrylic acid $\left(\mathrm{Kp}_{11}\right)$ different acrylic acid concentration at $60^{\circ} \mathrm{C}$

Figure 40- Conversion-time dependence rate coefficient homopropagation of acrylic acid $\left(\mathrm{Kp}_{11}\right), 1500 \mathrm{~L} / \mathrm{mol}$.s Polymerization rate and inhibition time as a function of deoxygenation condition- No Deox 102

Figure 41-Conversion-time dependence of rate coefficient homopropagation of acrylic acid (Kp11) $5000 \mathrm{~L} / \mathrm{mol}$.s -Polymerization rate and inhibition time as a function of $15 \mathrm{~min}$ of deoxygenation condition 102

Figure 42- Conversion-time rate coefficient homopropagation of acrylic acid (Kp11) $2000 \mathrm{~L} / \mathrm{mol} . \mathrm{s}$-Polymerization rate and inhibition time as a function of $30 \mathrm{~min}$ of deoxygenation condition.

Figure 43- Conversion-time dependence of the rate coefficient homopropagation of acrylic acid (Kp11) $2000 \mathrm{~L} / \mathrm{mol}$.s -Polymerization rate and inhibition time as a function of $60 \mathrm{~min}$ of deoxygenation condition 103

Figure 44- Evolution of the gel fraction calculated using different numbers of generations $(n=1,23,4$ and 5$)$ 105 
Figure 45- Evolution of the gel fraction calculated with numerical fractionation technique with 5 generations using different reactivity ratios of PDBs 106

Figure 46- Evolution of the gel fraction calculated with numerical fractionation technique with 5 generations using different reactivity ratios of PDBs 106 


\section{TABLES LIST}

Table1-Copolymerization reaction.............................................43

Table 2- Schematic List of species present in the reaction.........................44

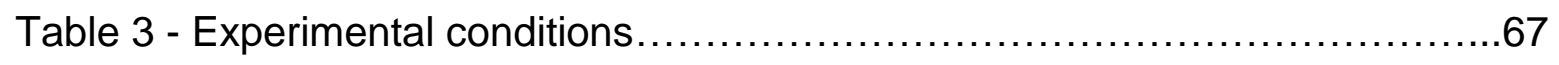

Table 4- Experimental conditions for the runs of the study of Inhibition period......68

Table 5- Kinetic parameters used in the preliminary analysis of the mathematical

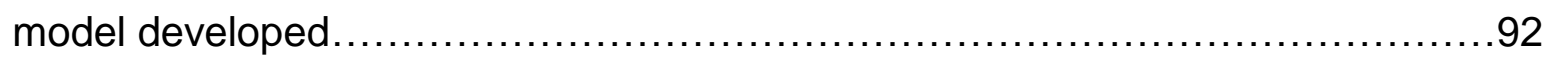

Table 6- Reported values of the termination rate constant (kp11) of acrylic acid in

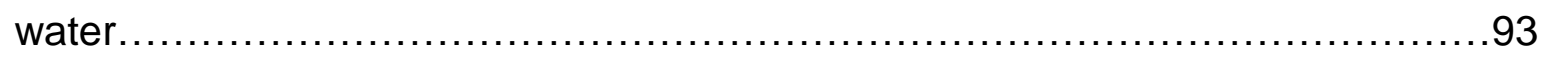

Table 7- Reported values of the propagation rate constant (kp11) of acrylic acid in

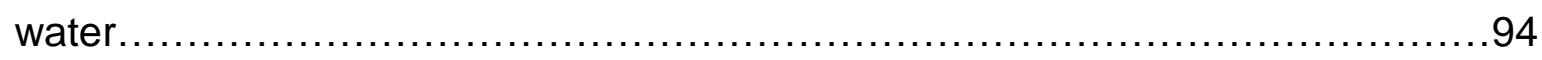




\section{NOMENCLATURE AND SIGLE LIST}
AA
Acrylic acid
TMPTA
Trimethylol propane triacrylate
PAA
Poly(acrylic Acid)
PDB
Pendent Double Bond
MWD
Molecular Weight Distribution
ODE
Ordinary Diferential Equation
TAO
Tetraallyloxy ethane
EGDMA
Ethylene glycol dimethylacrylate
FCC
Free-radical crosslinking copolymerization
MEHQ
Monomethyl ether of hydroquinone
MAA
Methacrylic acid
PSSH
Pseudo Steady State Hypothesis 


\section{SYMBOL LIST}

I: Initiator;

$\mathbf{D}_{\mathrm{i}}$ : Pendant double bond;

Ps: Dead Polymer containing "s" monomeric units;

$\mathbf{P}_{\mathrm{r}, \mathrm{i}}$ : Dead polymer containing pendant double bonds of type "i" and "r" monomeric units;

$\mathbf{R}_{\mathbf{0}}$ : Primary Radical (formed by the initiator decomposition);

$\mathbf{M}_{\mathrm{j}}$ : Monomer type $\mathrm{j}(\mathrm{j}=1$ : acrylic acid, $\mathrm{j}=2$ : trimethylolpropane triacrylate);

$\mathbf{R}_{r, i}:$ Polymeric radical of size " $r$ " and type "i" ( $\left.i=1: i=2: i=3: i=4: i=5\right)$;

S: Inert Species;

$P_{\mathbf{H}}=$ Hydrogen Potential

$\mathbf{k}_{\mathbf{d}}$ : Rate constant for the initiator decomposition $\left(\mathrm{s}^{-1}\right)$;

$\mathrm{K}_{\mathrm{i}, \mathrm{j}}$ : Rate constant of initiation of monomer or crosslinker agent $\left(\mathrm{L} \cdot \mathrm{mol}^{-1} \cdot \mathrm{s}^{-1}\right)$;

$\mathbf{K}_{\mathrm{p}, \mathrm{j} \mathrm{j}}$ : Rate constant of propagation of a radical of the type "i" and a monomer of type "j" (L.mol $\left.{ }^{-1} \cdot \mathrm{s}^{-1}\right)$;

$\mathrm{K}_{\mathrm{fr}, \mathrm{i}}$ : Rate constant of chain transfer of a radical of the type "i" $\left(\mathrm{L} \cdot \mathrm{mol}^{-1} \cdot \mathrm{s}^{-1}\right)$;

$\mathbf{k}_{\mathrm{h}}$ : Rate constant of the hydrogen abstraction reaction $\left(\mathrm{L} \cdot \mathrm{mol}^{-1} \cdot \mathrm{s}^{-1}\right)$;

$\mathrm{K}_{\mathrm{tc}}$ : Rate constant of termination by combination $\left(\mathrm{L} \cdot \mathrm{mol}^{-1} \cdot \mathrm{s}^{-1}\right)$

$\mathrm{K}_{\mathrm{td}}$ : Rate constant of termination by disproportionation $\left(\mathrm{L} \cdot \mathrm{mol}^{-1} \cdot \mathrm{s}^{-1}\right)$;

$\mathrm{K}_{\mathrm{l}}$ : Constant initiation;

$\mathrm{R}_{0}$ : Concentration of primary radicals;

M: Concentration monomer; 
$\mathrm{K}_{\mathrm{p}}$ : Constant propagation;

$Y_{0}$ : Moment of order zero for total radicals;

V: Volume of the reaction medium;

$\rho_{u}$ : Density of the monomer unit $(\mathrm{g} / \mathrm{L})$;

$\rho_{\mathrm{M}}$ : Density of the monomer $(\mathrm{g} / \mathrm{L})$.

$\mathrm{C}_{\mathrm{l}}$ : molar concentration to Sodium Persulfate (moles/liter)

$\mathrm{C}_{\mathrm{M} 1}$ : molar concentration to Acrylic acid (moles $\left./ \mathrm{ml}\right)$

$\mathrm{C}_{\mathrm{M} 2}$ : molar concentration to Trimethylolpropanetriacrylate (moles/liter)

$\mathrm{C}_{\mathrm{D} 3}$ : molar concentration to Pendant double bond (moles/liter)

$\mathrm{C}_{\mathrm{D} 4}$ : molar concentration to Pendant double bond (moles/liter)

$\mathrm{q}_{\mathrm{e}}$ : Feed Flow Rate (L/min)

$m_{1}=$ weight of the empty Beaker flask $(g)$,

$\mathrm{m}_{2}=$ weight of the flask with hydroquinone solution $(\mathrm{g})$

$m_{3}=$ weight of the flask with the sample taken from the reactor plus $H Q(g)$,

$\mathrm{m}_{4}=$ weight of the flask with the dry sample $(\mathrm{g})$,

$\mathrm{f}_{\text {hydroquinone }}=$ weight fraction of the hydroquinone solution added to the sample

$\mathrm{f}_{\text {span60 }}=$ weight fraction of Span 60 in the formulation,

$f_{\text {acrylic acid }}=$ weight fraction of acrylic acid in the formulation.

$m_{5}=$ weight of the empty filter paper $(\mathrm{g})$,

$m_{6}=$ weight of the initial dry polymer sample and the filter paper $(\mathrm{g})$,

$m_{7}=$ weight of the final dry sample after extraction and filter paper $(g)$. 


\section{SUMMARY}

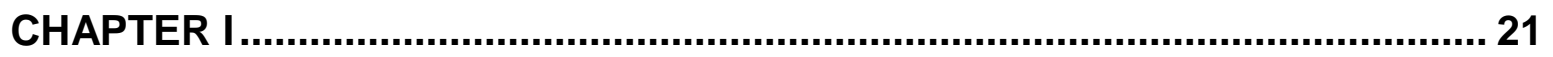

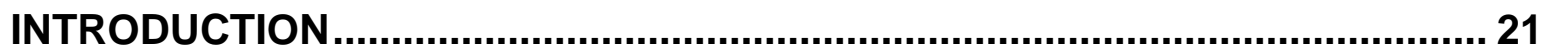

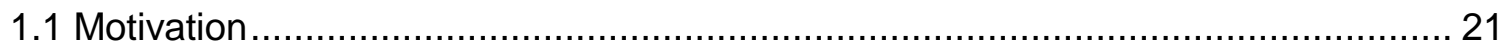

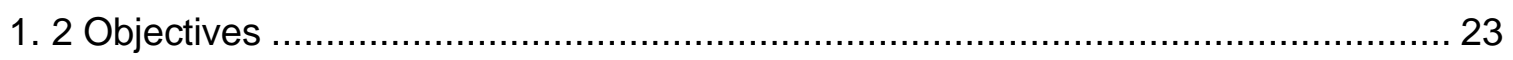

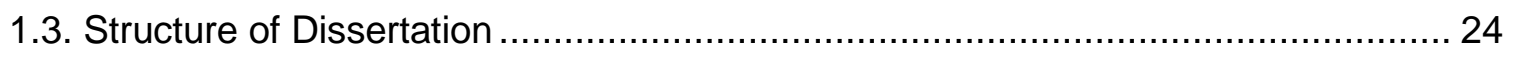

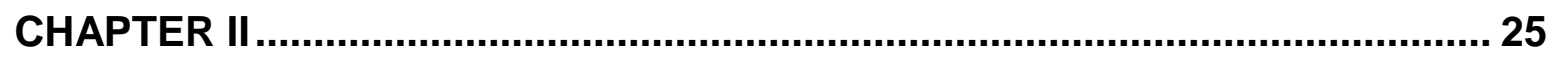

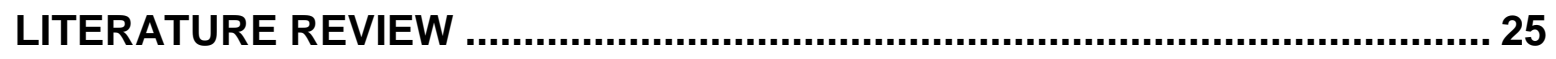

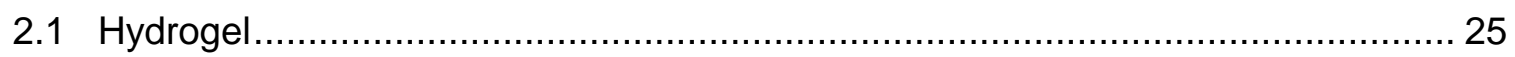

2.2 Crosslinking kinetics in poly(acrylic acid) networks ........................................... 26

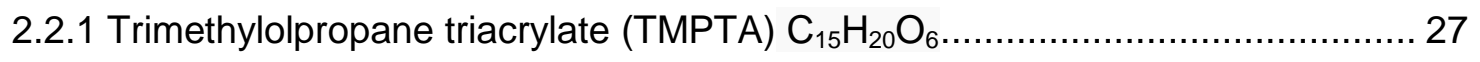

2.2.2 Glyoxal bis(diallyl acetal) or 1,1,2,2-Tetraallyloxyethane (TAO) $\mathrm{C}_{14} \mathrm{H}_{22} \mathrm{O}_{4} \ldots \ldots .27$

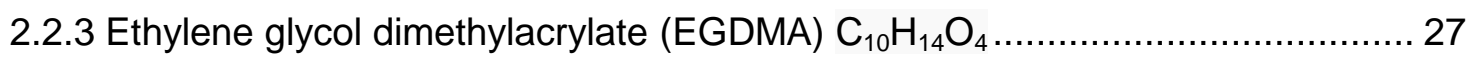

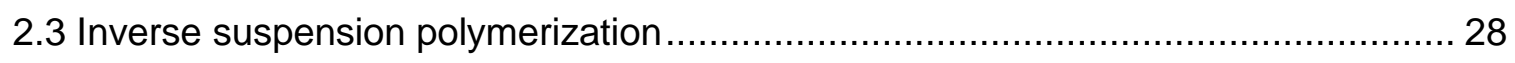

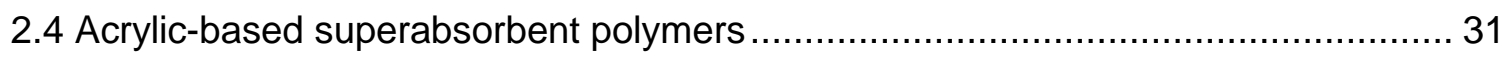

2.5 Kinetic modeling studies on the synthesis of superabsorbent polymers.................. 32

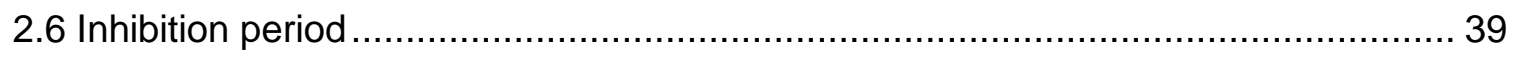

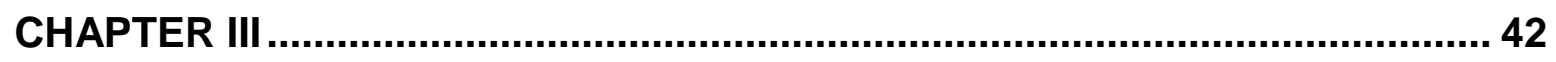

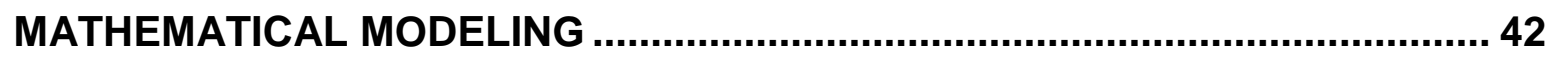

3.1 Polymerization Mechanism and Species Considered ........................................... 42

3.2 Establishing the assumptions to the developed model.......................................... 45

3.3 Determination of the kinetic pseudo-rate constants .............................................. 46

3.3.1 Pseudo kinetic constant of propagation monomers ....................................... 47

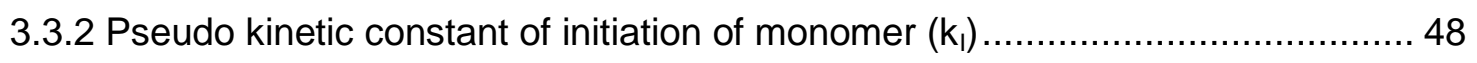

3.3.3 Pseudo kinetic constant of initiation of pendant double bond $\left(\mathrm{k}_{\mathrm{ID}}\right) \ldots \ldots \ldots \ldots \ldots \ldots . . . . . . . .49$

3.3.4 Pseudo kinetic constant of propagation of Pendant double bond $\left(\mathrm{K}_{\mathrm{PD}}\right)$........... 49 
3.3.5 Calculating the mole fraction of radicals of type "i" ................................. 51

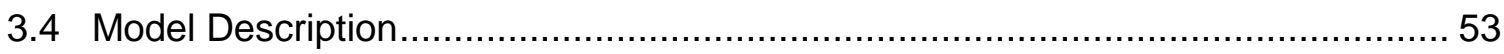

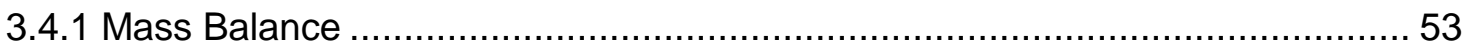

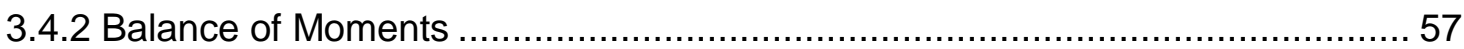

3.4.3 Numerical Fractionation Technique ....................................................... 60

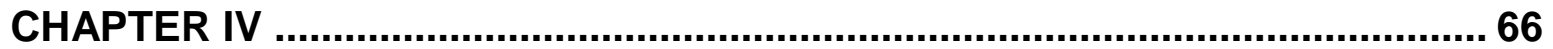

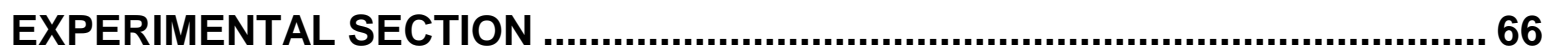

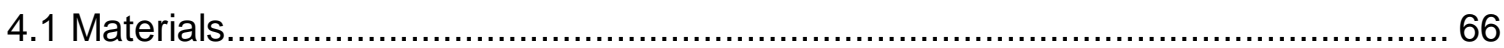

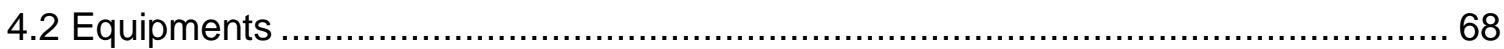

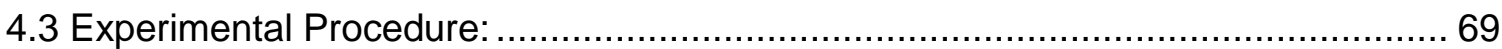

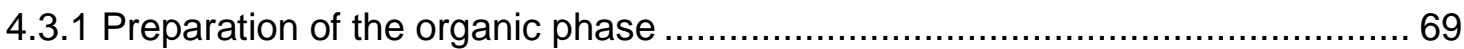

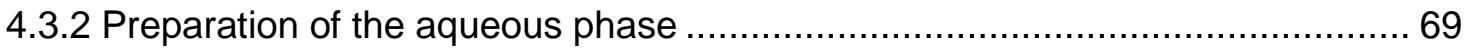

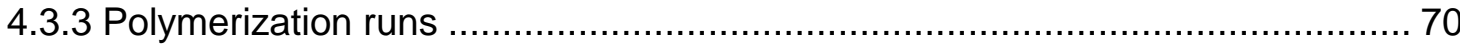

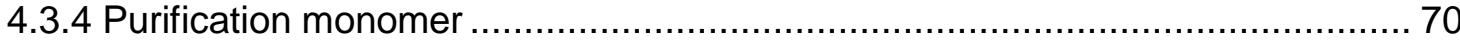

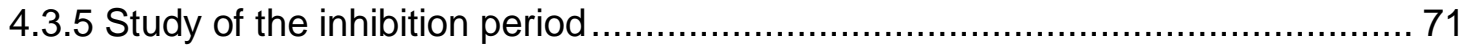

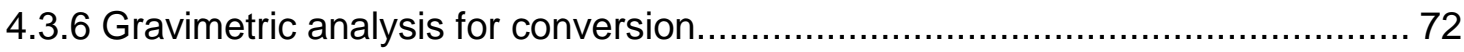

4.3.7 Determination of gel fraction by Soxhlet system ................................ 74

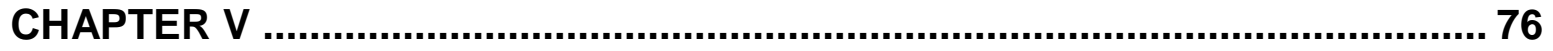

RESULTS AND DISCUSSIONS ....................................................................... 76

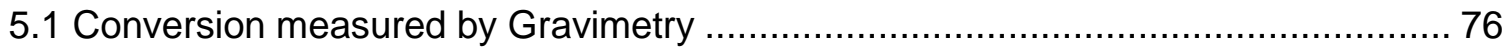

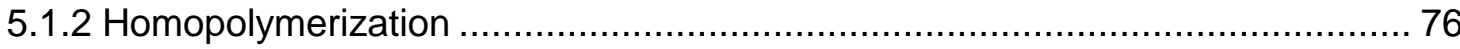

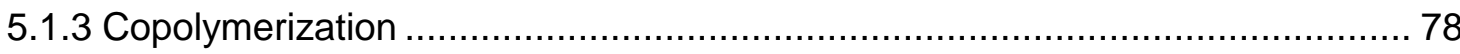

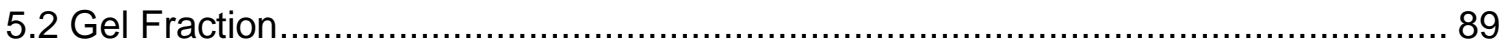

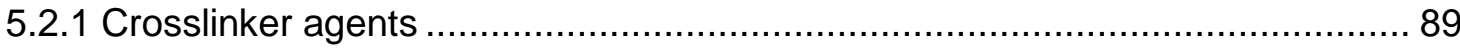

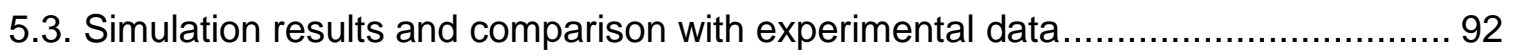

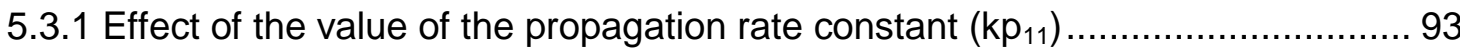




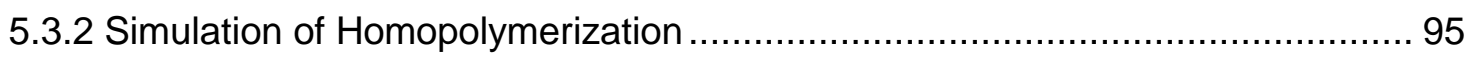

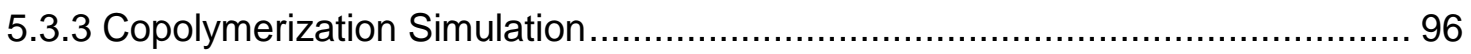

5.4 Analysis of the dynamics of gelation by the numerical fractionation technique ..... 104

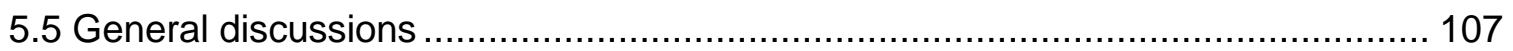

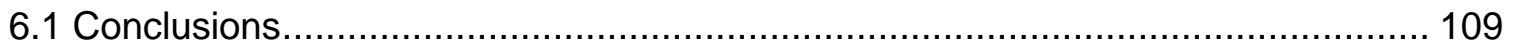

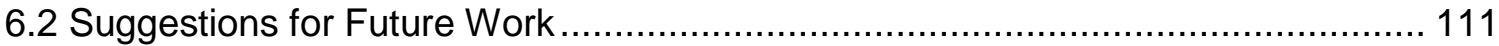

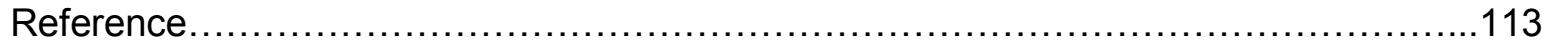

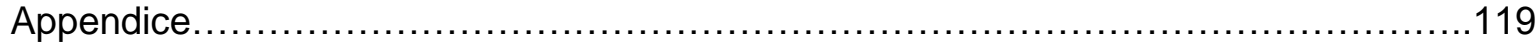




\section{CHAPTER I}

\section{INTRODUCTION}

This chapter presents the general considerations and motivation for the study, the objectives, and the structure of the dissertation.

\subsection{Motivation}

The synthesis and application of hydrogels have received special attention of researchers in recent years.

Hydrogels are three-dimensional chains of hydrophilic polymers or copolymers; despite their affinity for water, they are insoluble due to the presence of crosslinking in their structure, which characterizes them as polymer networks.

Hydrogels have been used in the sanitary industry, agriculture, environment, separation procedures and other operations of chemical engineering. They have also been used in clinical practice and experimental medicine for a wide range of applications, including tissue engineering and separation of biomolecules, among others. However, the application that has raised more interest is the controlled release of drugs.

The modeling of the hydrogels polymerization allows systematize the knowledge of the reaction mechanism. The importance of developing the models and improve the ability to predict, and therefore to control the reaction rates and the molecular structure of the polymer formed, are key aspects in the design and operation of polymerizing reactors.

In relation to mathematical modeling, currently there is a growing need for a more detailed understanding of the phenomena taking place in the polymer 
reactor. One quantitative form of this process understanding is the mathematical model, which can represent the detailed behavior of the polymerization process. The mathematical model is an invaluable tool for developing the optimal design and optimal control system for these reactors.

The copolymerization with polyvinyl acrylic monomers in aqueous phase has a complex kinetics that have been studied in recent years, the influence of factors such as temperature and $\mathrm{pH}$ on the reaction rate of these chemical systems. Due to lack of mechanistic understanding of this process, the literature still has little information regarding the speed of the reactions involved in the polymerization of hydrogels.

Suspension polymerization has been the focus of much attention due to its easy temperature control, low viscosity of the dispersion, low levels of impurities within the polymer product, and low separation cost, when compared with bulk and solution polymerization processes.

The advances in industrial production of polymers occurred in an accelerated manner. Synthetic polymers present advantages such as versatility in terms of final properties (e.g., glass transition temperature and modulus of elasticity), which can be modified in function of the process variables (e.g., concentration of reactants, additives and temperature). Many polymers are produced and sold without a clear, detailed understanding on the polymer structure or on the reactions involved in their synthesis. For instance, in the controlled radical copolymerization process, some open questions are the need to define the mechanism involved, the presence of secondary reactions; the effects caused by the addition of controlling agent on the gel point and on the polymer properties produced; the formation of cyclizations and their effects on the final product properties.

In the present work, the production of hydrogels of poly (acrylic acid) was experimentally studied using the inverse suspension polymerization process. The present work contributes as an effort improve the understanding of the 
polymerization process of the synthesis of hydrogels, by combine a set of experiments and the interpretation of the results by a mathematical model of the process.

In this work, a kinetic model based on population balance was developed to simulate the inverse-suspension polymerization process for producing hydrogels of acrylic acid and trimethylolpropane triacrylate. The experiments were carried out in a semibatch mode with control of the process variables. The sensitivity of the copolymerization of acrylic acid and trimethylolpropane triacrylate to the presence of dissolved oxygen in the reaction medium was analyzed to verify possible action as inhibitor or retardant of the polymerization.

The purpose of this study is to better understand the kinetic mechanism, to evaluate kinetics parameters, as well as the effects of the operating conditions, for example, temperature or reagent concentrations during the polymerization process on the monomer conversion and gel fraction.

\section{2 Objectives}

\section{General objetive}

- To perform experiments of polymerization of acrylic acid / trimethylolpropane triacrylate (TMPTA) and measure process variables such as monomer conversion and gel fraction, modifying reagent concentrations of initiator and crosslinking, and evaluating effects on the reaction to different operating temperatures, feeding time, and presence/concentration of inhibitor.

\section{Specific objectives}

- To identify the phenomena involved in the production process of the hydrogels and interpret them in terms of the reaction mechanisms currently found in the literature, thus contributing to a better understanding of the polymerization 
process in terms of the fundaments (a mathematical model was used to assist in this study);

- To determinate the changes in gel fraction during the prolymerization process, by using the extraction method, and from experimental data, to analyze the evolution of the gel fraction using different number of generations in the technique of numerical fractionation and considering different values of kinetic constants;

- To verify the effectiveness of the cross-linking agents trimethylolpropane triacrylate (TMPTA), tetraallyloxyethane (TAO) and ethylenglicol dimetracrylate (EGDMA) used in the experimental study and compare the gel formation in the systems.

\subsection{Structure of Dissertation}

This dissertation is structured as described below.

In Chapter 2, a literature review on the subjects related to this work is presented, emphasizing some recent work in the area. It also describes some concepts that were used throughout the study.

Chapter 3 describes the mathematical model of the polymerization system studied.

Chapter 4 presents the experimental equipment and the methods employed in the experiments.

Chapter 5 presents the results of the experimental study, as well as, the simulation results.

Finally, Chapter 6 presents conclusions and recommendations and suggestions for further studies. 


\section{CHAPTER II}

\section{LITERATURE REVIEW}

This Chapter presents general information taken from the literature about the main topics treated in the dissertation. It includes the basic concepts about hydrogels and inverse suspension polymerization. Also, a literature review is presented on the different experimental studies and on the kinetic studies of polymerization processes used to obtain hydrogels.

\subsection{Hydrogel}

Hydrogel products constitute a group of polymeric materials, the hydrophilic structure of which renders them capable of holding, by swelling, large amounts of water in their three-dimensional networks. Extensive use of these products in a number of industrial and environmental areas of application is considered to be of prime importance such as pharmaceutical, biology, separation process etc.

The materials of interest in this brief review are primarily hydrogels, which are polymer networks extensively swollen with water.

Hydrogels can be divided into two categories based on the chemical or physical nature of the cross-link junctions. Chemically cross-linked networks have permanent bonds, while physical networks have nonpermanent connections that arise from either polymer chain entanglements or physical interactions such as ionic interactions, hydrogen bonds and dipole forces (Ahmed, 2013).

Hydrogels are usually prepared from polar monomers. According to their starting materials, they can be divided into natural polymer hydrogels, synthetic polymer hydrogels, and combinations of the two classes. From a preparative point of view, they can be obtained by graft polymerization, cross-linking polymerization, networks formation of water-soluble polymer, radiation-induced cross-linking, etc. There are many types of hydrogels; mostly, they are slightly cross-linked 
copolymers of acrylate and acrylic acid, and grafted starch-acrylic acid polymers prepared by inverse suspension, emulsion polymerization, and solution polymerization.

\subsection{Crosslinking kinetics in poly(acrylic acid) networks}

A cross-link is a bond that links one polymer chain to another. They can be covalent bonds or ionic bonds. "Polymer chains" can refer to synthetic polymers or natural polymers (such as proteins). When the term "cross-linking" is used in the synthetic polymer science field, it usually refers to the use of cross-links to promote a difference in the polymers' physical properties. When "crosslinking" is used in the biological field, it refers to the use of a probe to link proteins together to check for protein-protein interactions, as well as other creative cross-linking methodologies.

Cross-linking is used in both synthetic polymer chemistry and in the biological sciences. Although the term is used to refer to the "linking of polymer chains" for both sciences, the extent of crosslinking and specificities of the crosslinking agents vary. When cross links are added to long rubber molecules, the flexibility decreases, the hardness increases and the melting point increases as well.

Network formation in free-radical polymerization is a non-equilibrium process, namely, it is kinetically controlled, and therefore each primary polymer molecule experiences a different history of crosslinking.

Different kind of crosslinker can be used in order to produce hydrogels; two most used are trimethylolpropane triacrylate (TMPTA) and tetraallyloxyethane (TAO) and ethylene glycol dimethylacrylate (EGDMA). 


\subsubsection{Trimethylolpropane triacrylate (TMPTA) $\mathrm{C}_{15} \mathrm{H}_{20} \mathrm{O}_{6}$}

Trimethylolpropane triacrylate is a trifunctional monomer used in the manufacture of plastics, adhesives, etc. It is useful for its low volatility and fast cure response, and improves the properties of resistance against weather, chemical, water and abrasion. End products include alkyd coatings, compact discs, hardwood floors, concrete polymers, dental polymers, lithography, letterpress, screen printing, elastomers, automobile headlamps, acrylics and plastic components for the medical industry.

\subsubsection{Glyoxal bis(diallyl acetal) or $1,1,2,2-$ Tetraallyloxyethane (TAO) $\mathrm{C}_{14} \mathrm{H}_{22} \mathrm{O}_{4}$}

1,1,2,2-Tetraallyloxyethane is a tetrafunctional crosslinker used for different applications; it has a high functionality due to the presence of the double bonds, with variations in their reactivities. This crosslinking agent is used to prepare superabsorbent, hydrophilic gels that are able to retain, at a high absorption rate, huge amounts of water.

\subsubsection{Ethylene glycol dimethylacrylate (EGDMA) $\mathrm{C}_{10} \mathrm{H}_{14} \mathrm{O}_{4}$}

Ethylene glycol dimethylacrylate is a diester formed by condensation of two equivalents of methacrylic acid and one equivalent of ethyleneglycol. EGDMA can be used as crooslinker in free radical copolymerizations. When used with methyl methacrylate, it leads to gel point at relatively low concentrations because of the nearly equivalent reactivities of all the double bonds involved. 


\subsection{Inverse suspension polymerization}

Dispersion polymerization is an advantageous method for preparing polymer dispersions because the products are obtained as powder or microspheres (beads), and thus, grinding is not required. In the production of hydrogels, water-inoil (W/O) process is chosen instead of the more common oil-in-water $(\mathrm{O} / \mathrm{W})$ processes, and then the polymerization is referred to as "inverse suspension polymerization".

The dispersion is thermodynamically unstable and requires both continuous agitation and addition of a lipophilic-balanced (HLB) suspending agent.

In suspension polymerization, the drops are stabilized against coalescence by the addition of water-soluble polymers called stabilizers or protective colloids. One of the commonly used stabilizers is Span 60 (Ahmed, 2013).

The function of surface-active agents is to absorb onto the droplet interface and prevent other drops from approaching because of electrostatic and/or steric repulsion forces. This causes a reduction of immediate coalescence due to the increasing strength of the liquid film entrapped between two colliding drops. The presence of a protective film prolongs the contact time required for drop coalescence, thus, increasing the probability of drop separation by agitation (Ahmed, 2013).

The use of inverse suspension polymerization is a development relatively new. Inverse suspension polymerization is conducted by dispersing water-soluble monomers in a continuous organic phase. Thermodynamically, the dispersion is unstable and requires continuous stirring and adding of stabilizing agents. Initiation (production of free radicals) is usually done thermally or chemically, with chemical initiators such as azo-compounds or peroxide compounds. In the case of using a single initiator component, polymerization may be initiated by decomposition of the initiator in the organic phase, in the aqueous phase or in both phases, depending 
on how the initiator is partitioned into the two phases (Machado; Lima; Pinto, 2007).

Kalfas et al. (1993) carried out extensive studies of aqueous suspension homo- and copolymerizations with methyl methacrylate, styrene, and vinyl acetate in a lab-scale batch reactor. Simulation results from homogeneous and two-phase free-radical polymerization kinetic models, using physical and kinetic parameter values taken from the literature of bulk and solution polymerization were found to be in good agreement with batch suspension experimental results.

Wang et al. (1997) studied the synthesis of water-superabsorbent sodium polyacrylate by inverse suspension polymerization, using Span60 as the dispersant, cyclohexane as the organic phase, $N, N^{*}$-methylene bisacrylamide as the crosslinking agent, and potassium persulfate as the initiator. The effect of reaction conditions such as reaction time, and concentrations of crosslinking agent, and dispersant on the swelling of deionized-water and saline solution, average particle size, and distribution of the sol-gel of the resin was discussed. The deionized-water and saline-solution swelling ratios of sodium polyacrylate prepared at proper conditions were $300-1200$ and 50-120, respectively; the numberaverage particle size was $10-50 \mathrm{~mm}$ and the weight fraction of gel was $20-85 \mathrm{wt} \%$.

Omidian et al. (1998) reported an exploratory investigation of the influence of cross-linking agents on the capacity for absorbing water and on the rate of absorption of acrylate polymers produced by using both inverse suspension and solution polymerization, which are the main process used industrially. They used a simple, small scale laboratory version of the polymerization part of this process, which permitted contact with air and evaporative losses, the effects of varying the heat input and the initiator concentration were explored. The presence of oxygen resulted in an inhibition period which prolonged the time for completing polymerization and consequently increased evaporative losses of water. The swelling was highest for the products obtained under conditions of short reaction times. Long reaction times resulted in long inhibition periods, runaway 
polymerization and low swelling. These effects were accounted for in terms of oxygen participation in the polymerization and extensive losses of water as the solvent.

Mayoux et al. (2000) studied the crosslinked poly(acrylic acid) synthesized by inverse suspension polymerization. This process was investigated to determine the influence of different parameters like temperature, stirring speed, solution $\mathrm{pH}$, and crosslinker concentration and to obtain the best control of the kinetics. An aqueous phase containing partially neutralized acrylic acid, crosslinking agent, and initiator agent was dispersed in an organic phase and stabilized by a surfactant. The inverse suspension was carried out in heptane as the organic phase with a different ratio of neutralization of the monomer, different crosslinker concentrations, and several stirring speeds. The polymerization was initiated by potassium persulfate $\left(\mathrm{K}_{2} \mathrm{~S}_{2} \mathrm{O}_{8}\right)$ with $\mathrm{N}-\mathrm{N}^{\prime}$-methylenebisacrylamide (MBAC) as the crosslinker and sorbitan monooleate as the surfactant. The influence of several parameters on the bead size and the swelling capacity was investigated. Particle diameters ranged from 10 to $130 \mu \mathrm{m}$. The kinetic results obtained by differential scanning calorimetry showed that conversion and polymerization rates are a function of the solution $\mathrm{pH}$, and they decreased when the concentration of the crosslinking agent MBAC was higher than $7.5 \%$.

Choudhary (2009) carried out inverse suspension polymerizations in a oneliter glass reactor to produce superabsorbent polymers (SAPs) based on acrylic monomers for hygiene applications. Strongly water-absorbing polymers, based on acrylic acid, sodium acrylate were prepared by copolymerization using potassium persulfate as initiator and $\mathrm{N}^{-} \mathrm{N}^{\prime}$ methylene-bisacrylamide (MBA) as crosslinking agent. The effect of varying monomer, crosslinker, initiator, dispersant concentration, reaction time and degree of neutralization, on absorption capacities was investigated. The continuous hydrocarbon phase was taken as 50:50 wt\% mixture of $n$-heptane and cyclohexane (aliphatic-alicyclic) because the availability of crosslinker in the aqueous phase is controlled by the partition coefficient of the 
crosslinker between the aqueous phase and the continuous hydrocarbon phase. The SAPs were evaluated for their free absorption capacities in distilled water, saline solution $(0.9 \mathrm{wt} \% \mathrm{NaCl})$, and also absorption under load (AUL). The experimental results show that these SAPs have good absorbency both in water and in $\mathrm{NaCl}$ solutions. It was observed that SAP synthesized from acrylic acid with about $70 \%$ degree of neutralization, containing $1 \mathrm{wt} \%$ cross-linker, and $0.5-1.0 \mathrm{wt} \%$ initiator concentration with $10 \mathrm{wt} \%$ dispersant exhibited absorption capacities in water, saline solution as $220 \mathrm{~g} / \mathrm{g}, 70 \mathrm{~g} / \mathrm{g}$, respectively.

\subsection{Acrylic-based superabsorbent polymers}

From a material resource point of view, superabsorbent polymers (SAPs) can also be divided into natural macromolecules, semi-synthetic polymers, and synthetic polymers. From a preparation point of view, they can be synthesized by graft polymerization, cross-linking polymerization, networks formation of watersoluble polymer and radiation cross-linking, etc. There are many types of SAPs presently in the market, mostly they are lightly cross-linked copolymers of acrylate and acrylic acid and grafted starch-acrylic acid polymers prepared by inverse suspension, emulsion polymerization, and solution polymerization. There are some examples of different studies.

Cutié et al. (1997) described the simplest polymerization kinetic model, which has a first-order dependence in monomer and half order in initiator. The isothermal polymerization $\left(55^{\circ} \mathrm{C}\right)$, of acrylic acid in water was monitored as a function of concentration, degree of neutralization and initiator level to define a kinetic model and to obtain accurate values for the rate constants.

The thesis of Souza (2013) focused on the experimental study of the synthesis of hydrogels of poly(acrylic acid), as well as its mathematical representation by building a kinetic model. For this purpose, experiments of acrylic 
acid homopolymerization and copolymerization of acrylic acid and trimethylpropane triacrylate in aqueous solution using sodium persulfate as initiator were performed. Conversion, gel fraction and quantification of pendant double bonds were determined using gravimetric techniques, extraction with water and titration respectively. A mathematical model was developed, in order to achieve a better understanding of the process.

\subsection{Kinetic modeling studies on the synthesis of superabsorbent polymers}

Few recent studies were found in the literature on kinetic modeling of the reaction of inverse suspension polymerization for producing hydrogels.

Arriola et al. (1997) studied the superabsorbent polymer gels prepared with acrylate crosslinkers such as TMPTA (Figure 1). They consisted of a very heterogeneously network of highly crosslinked, high molecular weight poly(acrylic acid) formed during the early stages of the polymerization, and lower molecular weight chains that form during the middle of the polymerization bridging through the pendant vinyl groups and containing a greater distance between crosslinking sites than chains formed initially and grafted polymer formed (especially) toward the end of the polymerization.

Figure 1. Reaction between acrylic acid and trimethylolpropane triacrylate to prepare the superabsorbenthydrogels.

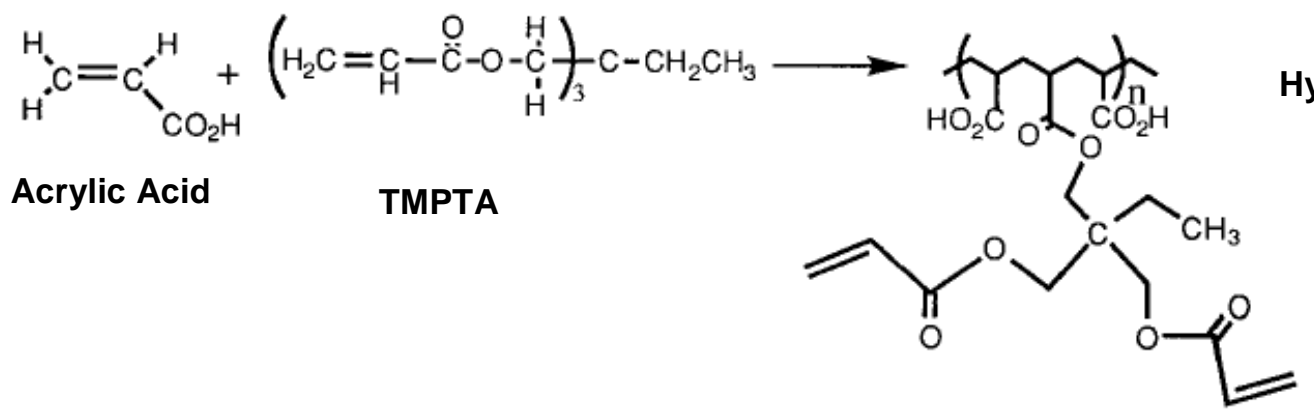


Arriola et al. (1997) evaluated the reactivity ratio for acrylic acid, varied from 0.31 (65\% neutralization) to 0.77 (non-neutralized). The reactivity ratio was affected by the percent solids (solvent effect), but was insensitive to temperature over the range of $55-80^{\circ} \mathrm{C}$. It was observed that all of the double bonds of TMPTA were incorporated into gel network as opposed to prior models predicting only two bonds reacting. The reported inefficiency of TMPTA is postulated to be caused by a solubility problem in the monomer mixture. Very low levels of extractables were found in the products even though the crosslinker was consumed by $70 \%$ conversion. Based on these data, they proposed that a major component of the gel network is graft polymer that forms late in the polymerization onto the crosslinked gel formed earlier.

Cutie et al. (1997) used nuclear magnetic resonance (NMR) to monitor the polymerization rates in situ. This method made it possible to obtain isothermal data during exothermic conditions of up to $2 \%$ conversion/min in $5 \mathrm{~mm}$ tubes. The $5 \mathrm{~mm}$ diameter NMR tubes were loaded via a glass pipette and deoxygenated with nitrogen. The results showed that the rate of incorporation of the crosslinker (TMPTA) was, under all conditions, much faster than the rate of polymerization of acrylic acid, the rate of incorporation of crosslinker resulting in earlier depletion from the monomer mixture, was faster in the more neutralized systems.

McKenna et al. (2000) investigated the inverse suspension of partially neutralized acrylic acid under variations of initiator and surfactant concentrations; they used data taken at low initiator concentration and maximum stirring rate to evaluated some kinetic constants and compared with literature values.

Costa et al. (2003) presented a kinetic approach for modeling irreversible non-linear polymerizations. Their model utilizes the numerical fractionation technique and is capable of predicting a broad range of distributional properties both for pre- and postgel operating conditions as well as polymer properties such 
as the crosslink density and branching frequency. Mass balance equations in terms of the moment generating function of the distribution of mole concentrations of polymeric species for free radical copolymerizations of mono/divinyl monomers could be numerically solved after gel point. They observed that the predictions by the pseudo-kinetic method are reasonable only when equal reactivity of double bonds prevails, causing early gelation in the batch reactor. The mathematical model for the crosslinking copolymerization of a vinyl and a divinyl monomer was developed and applied to the case of polymerization of methyl methacrylate and ethylene glycol dimethacrylate in a batch reactor. Model results compared favorably to the experimental data of $\mathrm{Li}$ et al. (1989) for the system investigated and confirmed the experimental findings of $\mathrm{Li}$ et al. (1989) that either the increase of CTA concentration or the decrease of divinyl monomer delays the onset of gelation. The effects of these variables on the crosslink densities are also demonstrated using this model. After gelation, the decrease in sol crosslink density is faster at higher levels of divinyl monomer, while gels of higher crosslink densities are obtained at higher levels of divinyl monomer.

Harrisson et al. (2003) evaluated the rate constants of propagation and termination of methyl methacrylate (MMA) in the ionic liquid 1-butyl-3methylimidazolium hexafluorophosphate using the pulsed laser polymerization technique across a range of temperatures. Arrhenius parameters were calculated for the rate of propagation at ionic liquid concentrations of 0,20 , and $50 \% \mathrm{v} / \mathrm{v}$. The decrease in activation energy leads to large increases in the rate of propagation. In addition, the rate of termination decreases by an order of magnitude as the ionic liquid concentration is increased to $60 \% \mathrm{v} / \mathrm{v}$. The increase in propagation rate was attributed to the increased polarity of the medium, while the decrease in the termination rate is due to its increased viscosity.

Rintoul et al. (2005) studied the reactivity ratio of polar monomers such as acrylic acid in free radical copolymerization. A strong impact on the reactivity ratios were identified for the $\mathrm{pH}$ and total monomer concentration. Specifically, at 
constant total monomer concentration of $0.4 \mathrm{~mol} / \mathrm{L}$ and $\mathrm{T}=313 \mathrm{~K}$, the reactivity ratio of acrylamide increases from 0.54 at $\mathrm{pH} 1.8$ to 3.04 at $\mathrm{pH} 12$. Contrarily, the reactivity ratio of acrylic acid decreases from 1.48 to 0.32 . Electrostatic effects to the variation of the degree of ionization of acrylic acid were primarily suggested to influence the kinetics. When the total monomer concentration increases from 0.2 to $0.6 \mathrm{~mol} / \mathrm{L}$ at constant $\mathrm{pH}=12$, the reactivity ratios of acrylamide and acrylic acid decrease from 4.01 to 2.13 and increase from 0.25 to 0.47 , respectively. Reduction of electrostatic repulsion between the ionized monomer acrylic acid and partially charged growing polymer chain ends due to higher ionic strength at higher total monomer concentration serves as explanation of the effect.

The thesis of Haque (2010) presented an experimental investigation performed to study the kinetics of copolymerization of monomers in aqueous and alcoholic media by considering factors such as type of initiator and solvent, and $\mathrm{pH}$, in order to determine how these affect the reactivity ratios of these monomers. Reactivity ratios were determined by non-linear least squares (NLLS) and the error-in-variables-model (EVM) techniques and full conversion range kinetic investigations were carried out to confirm these values.

Gonçalves et al. (2011) presented a kinetic model for gelling free radical polymerization based upon population balance equations of generating functions, and applied this model to predict the variations, in a batch reactor, of properties such as the weight fraction of gel and the average molecular weights of the soluble fraction. Simulations were carried out considering the synthesis of polyacrylic acid gels with a trifunctional crosslinker (TMPTA used as case study) at an initial mole fraction in the monomer mixture of $0.0025 \%$ (around the lower limit used in practice). In these simulations, three different values of the rate coefficient for the homopropagation of acrylic acid (kp1) were considered, in a range that is plausible for this monomer under these particular conditions. Predictions were used to evaluate the dependence of the dynamics of gelation on the following kinetic/operation parameters: 
- Propagation rate coefficient of monovinyl monomer (acrylic acid);

- Reactivity ratio of the pendant double bonds of the crosslinker;

- Initial mole fraction of the crosslinker;

- Functionality of the crosslinker (bi-, triand, tetrafuncional were considered).

Kinetic parameters (propagation and termination rate coefficients) for this copolymerization system varies with different synthesis conditions (temperature, concentration, $\mathrm{pH}$, ionic strength, etc), showing the following effects:

- Monomer/solvent concentration ratio with non-ionic systems. A decrease of about one order of magnitude in $\mathrm{kp}$ was observed upon increasing monomer concentration.

- Degree of ionization. At low monomer concentration, a decrease in $k p$ of about one order of magnitude was measured when the degree of ionization was changed from 0 to $100 \%$.

- Opposite variations were observed when the two effects (monomer concentration and ionization) are present: a weaker drop of $\mathrm{kp}$ with monomer concentration was found when the monomer is partially ionized. For a fully ionized monomer, $\mathrm{k}_{\mathrm{p}}$ increases when monomer concentration is also increased. Occurrence of Trommsdorff effect is another issue complicating the kinetics of these polymerization systems.

The above observations show that is difficult to establish a fully reliable set of kinetic parameters valid for the different conditions to be considered in the synthesis of water soluble homopolymers based on acrylic acid.

Simulations like those presented in Figure 2 and Figure 3 can be used to estimate the reactivity of PDBs using experimental measurements of the dynamics of gel formation. The effect of the initial mole fraction of crosslinker (Yc) on the dynamics of the weight fraction of gel is illustrated in Figure 4. This variable can be readily used to manipulate the properties of the final products, as depicted in that figure. Simulations for $Y_{c}$ ranging from the lower limit used in practice (around $0.0025 \%$ ) to ten times this value show the change of $\mathrm{Wg}$ from around 0.4 to 1 . 
Figure 2. Predicted dynamics of the weight fraction of gel $(\mathrm{wg})$ and monomer conversion. Effect of reactivity of the pendant double bonds

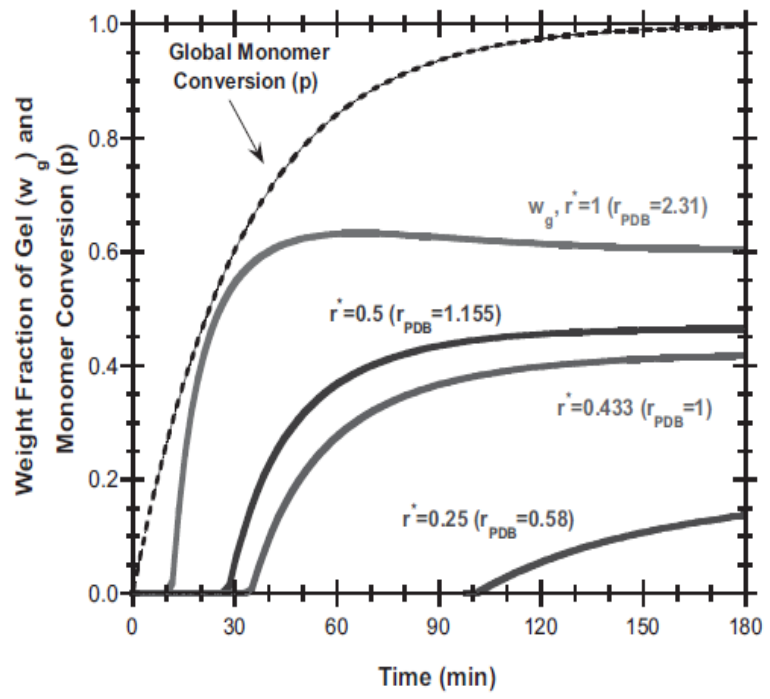

Ref: Gonçalves et al. (2011)
Figure 3. Predicted dynamics of the weigth fraction of gel (wg). Effect of the initial mole fraction of crosslinker.

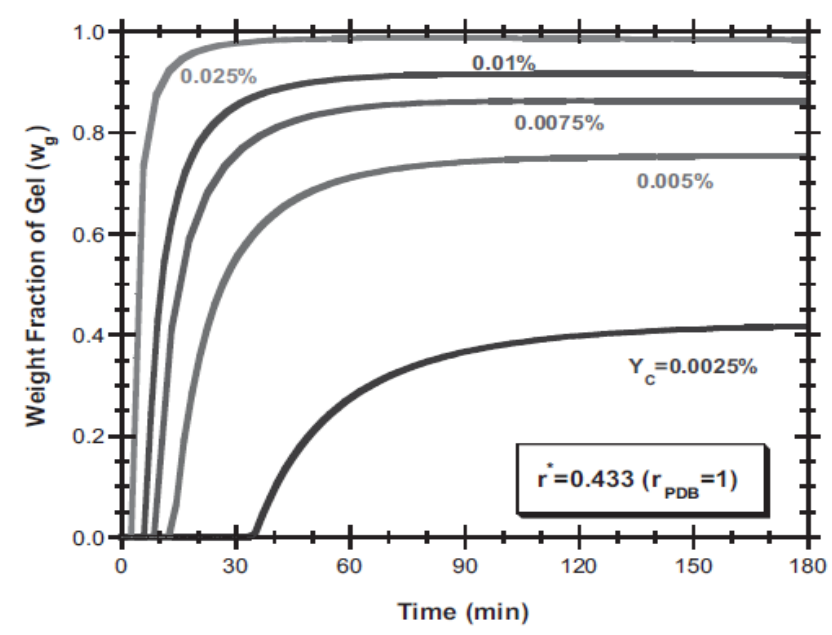

Under these conditions, gelation is predicted to occur within some hundredths of seconds as shown in Figure 4 and the weight fraction of gel in the polymer rises very fast to around 1. However, in practice, polymerization must be prolonged in order to reach high monomer conversion.

Kinetic gelation theories are able to deal with spatial heterogeneities resulting from topological constraints occurring with highly crosslinked networks but on the other hand present deficiencies with lightly crosslinked systems due to the failure to account for monomer and polymer mobility. 
Figure 4. Predicted time evolution of monomer conversion and $\mathrm{Wg}$

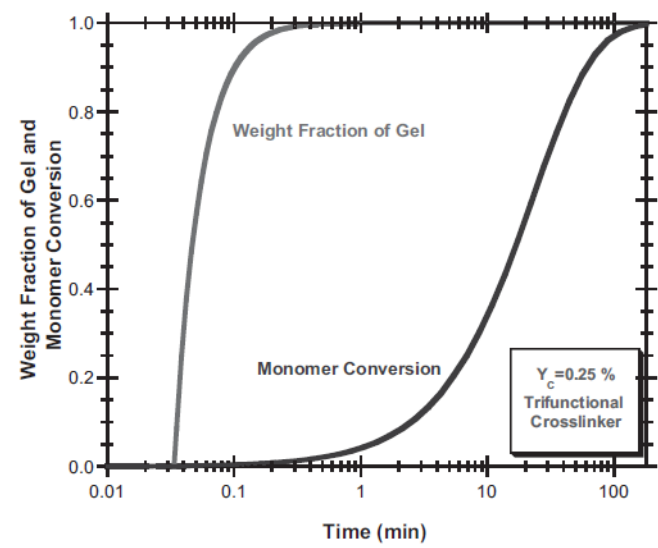

Ref: Gonçalves et al. (2011)
Figure 5. Predicted dynamics of the weigth fraction of gel with different crosllinker agents

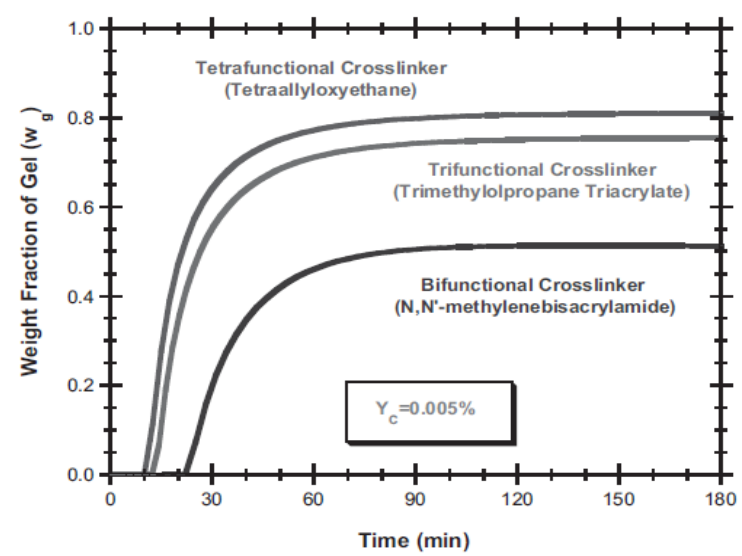

Ref: Gonçalves et al. (2011)

Another possible way to manipulate the dynamics of gelation is the choice of the functionality of the crosslinker, as shown in Figure 5. Three different synthesis processes, correspondent to the use of three crosslinkers with different functionalities (bi-, tri- and tetrafunctional, considering N,N'methylenebisacrylamide $\alpha=2$, trimethylolpropane triacrylate $\alpha=3$ and tetraallyloxyethane $\alpha=4$ ) were simulated as case studies. As expected, under the same conditions, the use of a crosslinker with higher functionally generates a higher amount of gel at a much higher rate. Substitution effects changing the reactivities of the different pendant double bonds of the crosslinker and possible low solubility of the crosslinker in the aqueous phase are some issues complicating the simple analysis performed.

The thesis of Gunter (2013) investigated the radical polymerization of methacrylic acid, acrylic acid and acrylamide in aqueous solution. Detailed kinetic models for both acrylic acid (AA), and methacrylic acid (MAA), developed applying the program PREDICITM, had a good representation of experimental conversion vs. time profiles and molar mass distributions. 


\subsection{Inhibition period}

Substances that may be present in the polymerization medium in very small quantities, causing a large decrease in the polymerization rate can be classified into two types according to their effectiveness: inhibitors and retarders. Inhibitors neutralize all free radicals, those come from the initiator, of the active centers of the chains or from the monomer. The polymerization is complete standstill until the inhibitor is consumed. Retardants are less effective inhibitors and neutralize only a fraction of the radicals. In this case, polymerization can occur, but at a slower speed. Some authors relate the mechanism responsible for inhibiting the deactivation of the centers of initiation or reduction in the rate of generation of these, while the delay associated with the interruption of chain propagation (Bamford, 1988). Same substance can act as an inhibitor, retarder or both simultaneously, depending on its concentration. The difference between inhibitors and retarders is therefore in magnitude, not as to the way in which the inhibition or delay occurs.

Cutie et al. (1997) studied the effect of the monomethyl ether of hydroquinone (MEHQ) on the polymerization of acrylic acid. The rate of polymerization was quantified at various levels of MEHQ by use of an in situ NMR technique. While oxygen acts as an inhibitor in acrylic acid polymerizations, MEHQ was shown to function as a retarder. The decrease in the rate of polymerization allowed the calculation of an inhibition constant for this system. MEHQ was found to remain in the polymerizing mixture throughout the course of the reaction, significantly reducing the rate of polymerization, but not reducing the molecular weight of the polymer.

Bunyakan et al. (1999) evaluated the isothermal acrylic acid polymerization by precipitation in toluene at various monomer and initiator concentrations over the temperature range of $40^{\circ} \mathrm{C}-50^{\circ} \mathrm{C}$. 2,2-Azobis (2,4-dimethyl-valeronitrile) was employed as a chemical initiator. The rate of polymerization was found to depend 
on the monomer and initiator concentration to the 1.7th and 0.6th orders, respectively. The greater than first order dependence on monomer concentration indicates a secondary, monomer-enhanced, decomposition step for the initiator molecules. The approximately half-order dependence of the rate of precipitation polymerization on the initiator concentration indicates that the chain termination process is predominantly bimolecular.

$\mathrm{Li}$ et al. (2006) studied the inhibitors MEHQ (monomethyl ether hydroquinone) and PTZ (phenothiazine) that are added to commercial acrylic acid to prevent its spontaneous polymerization during shipping and storage. Dissolved oxygen is also an strong inhibitor, and its presence in the solution enhances the inhibition effects of MEHQ. The authors developed a comprehensive mathematic model for the inhibition of acrylic acid polymerization and simulated the inhibition effects of oxygen and MEHQ on the polymerization of acrylic acid in batch and semi-batch processes. The key kinetic parameters were obtained from the literature or estimated from experimental data in the literature. The model was able to predict most of the effects of inhibitors. Although the literature data used did not show the synergistic inhibition effects of oxygen and MEHQ, the model was able to predict the effects. Simulation results showed that oxygen is a strong inhibitor in both batch and semi-batch reactors and does not act as a retarder as expected.

Lorber et al. (2010) presented an original tool to monitor polymerization kinetics. It consists in a droplet based millifluidic approach where the use of aqueous droplets of monomer can be seen as polymerization microreactors. Acrylic acid mixed with sodium persulfate at low $\mathrm{pH}$ was used as a fast and exothermic polymerization model. By using a nonintrusive spectroscopic system with a millifluidic system, they were able to safely investigate harsh polymerization conditions. As expected, polymerizations exhibited higher order with respect to monomer concentration than the usual first-order kinetics expected for ideal freeradical polymerization, and half-order dependence with respect to the initiator concentration. These results indicated the potential for further studies of 
polymerization reactions where detailed basic kinetic data must be acquired in conditions which cannot be investigated by using conventional batch glassware, i.e., high temperatures or concentrations. This versatile approach can also be used as an efficient high throughput screening tool. 


\section{CHAPTER III}

\section{MATHEMATICAL MODELING}

This Chapter presents the mathematical model used to interpret the experimental results of this dissertation for inverse-suspension polymerization of acrylic acid and trimethylolpropane triacrylate (TMPTA), a crosslinker with functionality 3 (number of active double bonds). This model was based on that developed in the thesis of Aguiar (2013) and also used in the work of Souza (2013).

\subsection{Polymerization Mechanism and Species Considered}

The mathematical model was developed in this research using the population balance for the different species present in the reactor.

The species considered are the following:

\section{I: Initiator;}

$\mathbf{D}_{\mathrm{i}}$ : Pendant double bond type i;

Ps: Dead Polymer containing "s" monomeric units;

$\mathbf{P}_{\mathrm{r}, \mathrm{i}}$ : Dead polymer containing pendant double bonds of type "i" and "r" monomeric units;

$\mathbf{R}_{\mathbf{0}}$ : Primary Radical (formed by the initiator decomposition);

$\mathbf{M}_{\mathrm{j}}$ : Monomer type $\mathrm{j}(\mathrm{j}=1$ : acrylic acid, $\mathrm{j}=2$ : trimethylolpropane triacrylate);

$\mathbf{R}_{r, i}$ : Polymeric radical of size " $r$ " and type "i" ( $\left.i=1: i=2: i=3: i=4: i=5\right)$;

S: Inert Species; 
Table 1 shows the reaction steps considered in the mechanism: decomposition of the initiator, initiation, propagation and termination of the chains, chain transfer to the polymer and the hydrogen abstraction. The kinetic scheme presented in Table 1 is a simplified description of the polymerization system that was here adopted in order to keep this presentation within a manageable size; however, this kinetic approach was conceived to deal with much more detailed descriptions of non-linear polymerization systems.

Table 1. Copolymerization reaction

\begin{tabular}{llll}
\hline Reaction & Mechanism & & \\
\hline Initiator Thermal Decomposition & $I \stackrel{k_{d}}{\longrightarrow} 2 R_{0}$ & & \\
Initiator & $R_{0}+M_{j} \stackrel{k_{i j}}{\longrightarrow} R_{1, j}$ & $1 \leq j \leq 2$ & \\
Monomer Propagation & $R_{r, i}+M_{j} \stackrel{k_{p, i j}}{\longrightarrow} R_{r+1, j}$ & $1 \leq i \leq 5, \quad 1 \leq j \leq 2$ \\
Crosslinker Initiator & $R_{0}+D_{i} \stackrel{k_{t, i}}{\longrightarrow} R_{r, i}$ & $3 \leq i \leq 4$ & \\
PDB Propagation (crosslinking) & $R_{r, j}+D_{i} \stackrel{k_{p i j}}{\longrightarrow} R_{r+1, j}$ & $1 \leq j \leq 5$ & $3 \leq i \leq 4$ \\
Hydrogen Abstration & $P_{s}+R_{0} \stackrel{k_{h}}{\longrightarrow} R_{s}+S$ & & \\
Chain Transfer & $P_{s}+R_{r, i} \stackrel{k_{r, i}}{\longrightarrow} R_{s}+P_{r, i}$ & $1 \leq i \leq 5$ & \\
Termination by combination & $R_{r, j}+R_{s, i} \stackrel{k_{k r}}{\longrightarrow} P_{r+s}$ & $1 \leq j \leq 5$ & $1 \leq i \leq 5$ \\
Termination by disproportionation & $R_{r, j}+R_{s, i} \stackrel{k_{u d}}{\longrightarrow} P_{r}+P_{s}$ & $1 \leq j \leq 5$ & $1 \leq i \leq 5$
\end{tabular}

$\mathbf{k}_{\mathbf{d}}$ : Rate constant for the initiator decomposition $\left(\mathrm{s}^{-1}\right)$;

$\mathbf{k}_{\mathrm{i}, \mathrm{j}}$ : Rate constant of initiation of monomer or crosslinker agent (L.mol $\left.{ }^{-1} \cdot \mathrm{s}^{-1}\right)$;

$\mathbf{k}_{\mathrm{p}, \mathrm{j} j}$ : Rate constant of propagation of a radical of the type "i" and a monomer of type "j" (L. mol $\left.{ }^{-1} \cdot \mathrm{s}^{-1}\right)$;

$\mathbf{k}_{\mathrm{fr}, \mathrm{i}}$ : Rate constant of chain transfer of a radical of the type "i" $\left(L \cdot \mathrm{mol}^{-1} \cdot \mathrm{s}^{-1}\right)$;

$\mathbf{k}_{\mathbf{h}}$ : Rate constant of the hydrogen abstraction reaction $\left(\mathrm{L} \cdot \mathrm{mol}^{-1} \cdot \mathrm{s}^{-1}\right)$;

$\mathbf{k}_{\mathbf{t c}}$ : Rate constant of termination by combination (L.mol ${ }^{-1} \cdot \mathrm{s}^{-1}$ )

$\mathrm{k}_{\mathrm{td}}$ : Rate constant of termination by disproportionation $\left(\mathrm{L} \cdot \mathrm{mol}^{-1} \cdot \mathrm{s}^{-1}\right)$; 
Table 2 presents a description of the different species, groups, radicals, monomers and pendant double bonds considered in the model.

Table 2 - Schematic list of species considered in the model.

\begin{tabular}{|c|c|c|}
\hline Symbol & Name & Species and group \\
\hline I & Initiator & \\
\hline $\mathbf{R}_{0}$ & Primary radical & \\
\hline$M_{1}$ & Acrylic Acid & \\
\hline $\mathbf{M}_{2}$ & TMPTA (Trimethylolpropanetriacrylate) & \\
\hline $\mathrm{D}_{3}$ & Pendant double bond (PBDs) & \\
\hline $\mathrm{D}_{4}$ & Pendant double bond (PBDs) & \\
\hline $\mathrm{U}_{1}$ & Polymeric units AA ( acrylic acid) & \\
\hline $\mathrm{U}_{2}$ & Polymeric unit AA (Acrylic Acid) & \\
\hline $\mathbf{R}_{1}$ & Radical AA (Acrylic Acid) & \\
\hline $\mathbf{R}_{\mathbf{2}}$ & $\begin{array}{c}\text { Radical TMPTA } \\
\text { (trimethylolpropanetriacrylate) }\end{array}$ & \\
\hline $\mathbf{R}_{3}$ & Radical $\mathrm{D}_{3}$ & \\
\hline $\mathbf{R}_{4}$ & Radical $\mathrm{D}_{4}$ & \\
\hline $\mathbf{R}_{5}$ & Backbone radical & \\
\hline
\end{tabular}




\subsection{Establishing the assumptions to the developed model}

The following simplifying assumptions were considered in the development of the kinetic model:

a) Pseudo Steady State Hypothesis (PSSH) for the radical species (i.e., the radicals are considered to be consumed almost instantly after they are produced, because of their very short lifetime; the net production rate of each radical species is nil);

b) Pseudo-homopolymerization approach (the kinetics of the copolymerization reactions are combined in proportion to the respective radical types and monomer types);

c) Each soluble polymer chain has only one radical center (mono-radical);

d) The closure equation of Saidel and Katz (Aguiar et al., 2014) was used to close the set of balance equations for the moments of the molecular weight distribution;

e) During each time integration step, fractions of each type of radical are considered to be constant. The rate of bimolecular reactions involving polymeric groups is independent of the size of the polymer chain;

f) The reactions occur only in droplets dispersed in the organic medium (the droplets behave or operate as a reactor); each monomer drop is considered as a mini-reactor in which a bulk polymerization takes place and the overall behavior of the reactor is the sum of every drop of behavior;

g) The drop density is constant (there are only changes in the volume during the feed of the aqueous phase in the polymerization process);

h) Negligible cyclization reactions (this assumption was adopted because the experimental quantification of the amount of double bonds for this systems is not easy) 


\subsection{Determination of the kinetic pseudo-rate constants}

Based on the assumptions and reaction mechanism for the polymerization of acrylic acid and TMPTA, two monomer species are considered, five types of polymeric radicals and two types of pendant double bonds, as shown in Table 2. In order to simplify the model equations for this reaction scheme, the assumption of pseudo-homopolymerization was adopted to determine the pseudo kinetic constants. These constants were determined for the reactions of initiation and propagation of monomers and pendant double bonds (Souza, 2013).

The mole fractions of the different radical types, of different monomer types, and of different pendant double bonds are defined, respectively, as:

$$
\begin{aligned}
f_{R i} & =\frac{R_{i}}{\sum_{j}^{5}\left[R_{j}\right]} \\
f_{M i} & =\frac{M_{i}}{\left(M_{1}+M_{2}\right)} \\
f_{D i} & =\frac{\left[D_{i}\right]}{[D]}
\end{aligned}
$$

$f_{S}=\frac{\left[D_{3}+D_{4}\right]}{\left[Q_{1}\right]}$

$[M]=\left[M_{1}\right]+\left[M_{2}\right]$

$$
[D]=\left[D_{3}\right]+\left[D_{4}\right]
$$




\subsubsection{Pseudo kinetic constant of propagation monomers}

Considering the reaction scheme given in Table 1 , the propagation rate is expressed by:

$$
\begin{aligned}
& R_{p}=R_{1}\left(k_{p 11} M_{1}+k_{p 12} M_{2}\right)+R_{2}\left(k_{p 21} M_{1}+k_{p 22} M_{2}\right)+R_{3}\left(k_{p 31} M_{1}+k_{p 32} M_{2}\right)+ \\
& +R_{4}\left(k_{p 41} M_{1}+k_{p 42} M_{2}\right)+R_{5}\left(k_{p 51} M_{1}+k_{p 52} M_{2}\right)
\end{aligned}
$$

For a homopolymerization, the propagation rate would be:

$$
R_{p}=k_{p} R M
$$

where $R$ is the total radical concentration, while $M$ is the total monomer concentration. Substituting Equation 7 into 8:

$$
\begin{aligned}
& k_{p} R M=R_{1}\left(k_{p 11} M_{1}+k_{p 12} M_{2}\right)+R_{2}\left(k_{p 21} M_{1}+k_{p 22} M_{2}\right)+R_{3}\left(k_{p 31} M_{1}+k_{32} M_{2}\right)+ \\
& +R_{4}\left(k_{p 41} M_{1}+k_{p 42} M_{2}\right)+R_{5}\left(k_{p 51} M_{1}+k_{p 52} M_{2}\right)
\end{aligned}
$$

Dividing both sides by $[R][M]$ :

$$
\begin{aligned}
& k_{p}=\frac{R_{1}}{R}\left(k_{p 11} \frac{M_{1}}{M}+k_{p 12} \frac{M_{2}}{M}\right)+\frac{R_{2}}{R}\left(k_{p 21} \frac{M_{1}}{M}+k_{p 22} \frac{M_{2}}{M}\right)+ \\
& +\frac{R_{3}}{R}\left(k_{p 31} \frac{M_{1}}{M}+k_{p 32} \frac{M_{2}}{M}\right)+\frac{R_{4}}{R}\left(k_{p 41} \frac{M_{1}}{M}+k_{p 42} \frac{M_{2}}{M}\right)+\frac{R_{5}}{R}\left(k_{p 51} \frac{M_{1}}{M}+k_{p 52} \frac{M_{2}}{M}\right) \Leftrightarrow \\
& k_{p}=f_{R 1}\left(k_{p 11} f_{M 1}+k_{p 12} f_{M 2}\right)+f_{R 2}\left(k_{p 21} f_{M 1}+k_{p 22} f_{M 2}\right)+f_{R 3}\left(k_{p 31} f_{M 1}+k_{p 32} f_{M 2}\right)+ \\
& +f_{R 4}\left(k_{p 41} f_{M 1}+k_{p 42} f_{M 2}\right)+f_{R 5}\left(k_{p 51} f_{M 1}+k_{p 52} f_{M 2}\right)
\end{aligned}
$$


Then the pseudo-rate constant of propagation can be calculated by:

$$
k_{p}=\sum_{i=1}^{5} \sum_{j=1}^{2} k_{p i j} f_{R i} f_{M j}
$$

where $f_{R i}$ and $f_{M j}$ are the molar fractions of the radical type i monomer of type $\mathrm{j}$, respectively.

\subsubsection{Pseudo kinetic constant of initiation of monomer $\left(k_{1}\right)$}

The rate of initiation of both monomers is:

$$
R_{I}=k_{I 1} M_{1} R_{0}+k_{I 2} M_{2} R_{0}
$$

For a homopolymerization, the initiation rate would be written as:

$$
R_{I}=k_{I} M R_{0}
$$

where $M$ is the monomer concentration. Substituting Equation 13 into 12,

$$
k_{I} M=k_{I 1} M_{1}+K_{I 2} M_{2}
$$

Dividing both sides by M:

$$
k_{I}=k_{I 1} \frac{M_{1}}{M}+K_{I 2} \frac{M_{2}}{M} \Leftrightarrow k_{I}=k_{I 1} f_{M 1}+k_{I 2} f_{M 2}
$$

We can re-write this equation in the form:

$$
k_{I}=\sum_{i=1}^{2} k_{I i} f_{M i}
$$

where $f_{M i}$ is the mole fraction of monomer type $\mathrm{i}$. 


\subsubsection{Pseudo kinetic constant of initiation of pendant double bond ( $\left.k_{I D}\right)$}

The rate of initiation of pending double bonds is expressed by:

$R_{I D}=k_{I D 3} R_{0} D_{3}+K_{I D 4} R_{0} D_{4}$

For a homopolymerization, the corresponding herefore, the initiation homopolymerization rate, for pendant double bond would be:

$$
R_{I R}=k_{I D} R_{0} Q_{1}
$$

Where $D$ is the concentration of pendant double bond. Substituting Equation 18 in 17 ,

$k_{I D} R_{0} Q_{1}=k_{I D 3} R_{0} D_{3}+k_{I D 4} R_{0} D_{4}$

Dividing both sides by $R_{0}$ and (D3 + D4)

$$
\begin{aligned}
& k_{I D} \frac{Q_{1}}{\left(D_{3}+D_{4}\right)}=k_{I D 3} \frac{D_{3}}{\left(D_{3}+D_{4}\right)}+k_{I D 4} \frac{D_{4}}{\left(D_{3}+D_{4}\right)} \Leftrightarrow k_{I D} \frac{1}{f_{s}}=k_{I D 3} f_{D 3}+k_{I D 4} f_{D 4} \Leftrightarrow \\
& k_{I D}=\left(k_{I D 3} f_{D 3}+k_{I D 4} f_{D 4}\right) f_{s}
\end{aligned}
$$

This equation can written as:

$$
k_{I D}=\left(\sum_{i=3}^{4} k_{I D i} f_{D i}\right) f_{s}
$$

where is the molar pending i.e., the double type is the molar fraction of pending double bond, the dead polymer fraction.

\subsubsection{Pseudo kinetic constant of propagation of Pendant double bond ( $\left.\mathrm{K}_{\mathrm{PD}}\right)$}

The propagation rate of the pendant double bonds is expressed as: 


$$
\begin{aligned}
& R_{P D}=R_{1}\left(k_{p 13} D_{3}+k_{p 14} D_{4}\right)+R_{2}\left(k_{p 23} D_{3}+k_{p 24} D_{4}\right)+R_{3}\left(k_{p 33} D_{3}+k_{p 34} D_{4}\right)+ \\
& +R_{4}\left(k_{p 43} D_{3}+k_{p 44} D_{4}\right)+R_{5}\left(k_{p 53} D_{3}+k_{p 54} D_{4}\right)
\end{aligned}
$$

While for a homopolymerization, the corresponding rate would be:

$$
R_{P D}=\left(k_{P D} R_{r} Q_{1}\right)
$$

where in $R_{r}$ represents the concentration of radicals size " $r$ " while $Q_{1}$ is the number of monomeric units in neutral polymer. Substituting Equation 23 into 22:

$$
\begin{aligned}
& k_{P D} \sum_{r=1}^{5} R_{r} Q_{1}=R_{1}\left(k_{p 13} D_{3}+k_{p 14} D_{4}\right)+R_{2}\left(k_{p 23} D_{3}+k_{p 24} D_{4}\right)+R_{3}\left(k_{p 33} D_{3}+k_{p 34} D_{4}\right)+ \\
& +R_{4}\left(k_{p 43} D_{3}+k_{p 44} D_{4}\right)+R_{5}\left(k_{p 53} D_{3}+k_{p 54} D_{4}\right)
\end{aligned}
$$

Dividing both sides by $\sum_{r=1}^{5} R_{r}$ e $\left(D_{3}+D_{4}\right)$ :

$$
\begin{aligned}
k_{P D} \frac{Q_{1}}{D_{3}+D_{4}} & =\frac{R_{1}}{\sum_{r=1}^{5} R_{r}}\left(k_{p 13} \frac{D_{3}}{D_{3}+D_{4}}+k_{p 14} \frac{D_{4}}{D_{3}+D_{4}}\right) \\
& +\frac{R_{2}}{\sum_{r=1}^{5} R_{r}}\left(k_{p 23} \frac{D_{3}}{D_{3}+D_{4}}+k_{p 24} \frac{D_{4}}{D_{3}+D_{4}}\right) \\
& +\frac{R_{3}}{\sum_{r=1}^{5} R_{r}}\left(k_{p 33} \frac{D_{3}}{D_{3}+D_{4}}+k_{p 34} \frac{D_{4}}{D_{3}+D_{4}}\right) \\
& +\frac{R_{4}}{\sum_{r=1}^{5} R_{r}}\left(k_{p 43} \frac{D_{4}}{D_{3}+D_{4}}+k_{p 44} \frac{D_{4}}{D_{3}+D_{4}}\right) \\
& +\frac{R_{5}}{\sum_{r=1}^{5} R_{r}}\left(k_{p 53} \frac{D_{3}}{D_{3}+D_{4}}+k_{p 54} \frac{D_{4}}{D_{3}+D_{4}}\right)
\end{aligned}
$$

$$
\begin{gathered}
k_{P D} \frac{1}{f^{\prime}{ }_{D}}=f_{R 1}\left(k_{p 13} f_{D 3}+k_{p 14} f_{D 4}\right)+f_{R 2}\left(k_{p 23} f_{D 3}+k_{p 24} f_{D 4}\right)+f_{R 3}\left(k_{p 33} f_{D 3}+k_{p 34} f_{D 4}\right) \\
\quad+f_{R 4}\left(k_{p 43} f_{D 3}+k_{p 44} f_{D 4}\right)+f_{R 5}\left(k_{p 53} f_{D 3}+k_{p 54} f_{D 4}\right) \\
k_{P D}=\left(\begin{array}{l}
f_{R 1}\left(k_{p 13} f_{D 3}+k_{p 14} f_{D 4}\right)+f_{R 2}\left(k_{p 23} f_{D 3}+k_{p 24} f_{D 4}\right)+f_{R 3}\left(k_{p 33} f_{D 3}+k_{p 34} f_{D 4}\right)+f_{D}^{\prime} \\
+f_{R 4}\left(k_{p 43} f_{D 3}+k_{p 44} f_{D 4}\right)+f_{R 5}\left(k_{p 53} f_{D 3}+k_{p 54} f_{D 4}\right)
\end{array}\right.
\end{gathered}
$$


Simplifying the equation:

$$
k_{P D}=\sum_{r=1}^{5} f_{R i}\left(k_{p i 3} f_{D 3}+k_{p i 4} f_{D 4}\right) f^{\prime}{ }_{D}
$$

where $f_{R i}$ and $f_{D j}$ are, respectively, the mole fractions of the polymeric radical of type "i" and the mole fraction of double bonds of type "j".

\subsubsection{Calculating the mole fraction of radicals of type "i"}

From a system of linear equations arising from the mass balance conducted for each group, it is possible to determine the fraction of radicals of type "i". Ortiz (2008) (apud Souza, 2013) disclosed a similar study using two types of radicals, which results in a less complex solution to the system of equations.

The mass balance for the polymer type groups $R_{1}$ is expressed below

$$
\begin{aligned}
& \frac{d R_{1}}{d t}=k_{I 1}\left[R_{0}\right]\left[M_{1}\right]-k_{p 12}\left[R_{1}\right]\left[M_{2}\right]+\sum_{i=2}^{5} k_{p i 1}\left[R_{i}\right]\left[M_{1}\right]-k_{p 11}\left[R_{1}\right]\left[M_{1}\right]-\sum_{i=3}^{4} k_{p 1 i}\left[R_{1}\right]\left[D_{i}\right]- \\
& -k_{f r 1} Q_{1}\left[R_{1}\right]-\sum_{j=1}^{5} k_{t}\left[R_{1}\right]\left[R_{j}\right]
\end{aligned}
$$

According to the pseudo-steady state:

$$
0=-k_{p 12}\left[R_{1}\right]\left[M_{2}\right]+\sum_{i=2}^{5} k_{p i 1}\left[R_{i}\right]\left[M_{1}\right]-k_{p 11}\left[R_{1}\right]\left[M_{1}\right]-\sum_{i=3}^{4} k_{p 1 i}\left[R_{1}\right]\left[D_{i}\right]-k_{f r 1} Q_{1}\left[R_{1}\right]
$$

Dividing both sides of the equation 28, is obtained:

$$
0=-k_{p 12} \frac{\left[R_{1}\right]}{\sum_{j=1}^{5} R_{j}} \frac{\left[M_{2}\right]}{(M+D)}+\sum_{i=2}^{5} k_{p i 1} \frac{\left[R_{i}\right]}{\sum_{j}^{5} R_{j}} \frac{\left[M_{1}\right]}{(M+D)}-\sum_{i=3}^{4} k_{p 1 i} \frac{\left[R_{1}\right]}{\sum_{j=1}^{5} R_{j}} \frac{\left[D_{i}\right]}{(M+D)}-
$$


$-k_{f r 1} \frac{\left[R_{1}\right]}{\sum_{j=1}^{5} R_{j}} \frac{Q}{(M+D)}$

and,

- $f_{R i}=\frac{\left[R_{i}\right]}{\sum_{j=1}^{5} R_{j}}$

- $f^{\prime}{ }_{i}=\frac{M_{i}}{M+D}$, for $i \leq 2$

- $f^{\prime}{ }_{i}=\frac{D_{i}}{M+D}$, for $3 \leq i \leq 4$

- $f^{\prime}{ }_{S}=\frac{Q_{1}}{M+D}$

$0=-k_{P 12} f_{R 1} f^{\prime}{ }_{2}+\sum_{r=2}^{5} k_{P i 1} f_{R i} f^{\prime}{ }_{1}-k_{P 11} f_{R 1} f^{\prime}{ }_{1}-\sum_{r=3}^{4} k_{P i 1} f_{R i} f^{\prime}{ }_{1}-k_{f r 1} f_{R 1} f^{\prime}{ }_{s}$

Developing the above equation, to obtain:

$0=f_{R 1}\left(-k_{P 12} f_{2}^{\prime}{ }_{2}-\sum_{i=3}^{4} k_{p 1 i} f_{1}^{\prime}-k_{f r 1} f_{S}^{\prime}\right)+f^{\prime}{ }_{1}\left(f_{R 2} k_{p 21}+f_{R 3} k_{p 31}+f_{R 4} k_{p 41}+f_{R 5} k_{p 51}\right)$

Following the same reasoning for the other extreme, one obtains the following equations

$$
\begin{aligned}
& 0=f_{R 2}\left(-k_{p 21} f_{2}^{\prime}-k_{p 23} f_{3}^{\prime}-k_{p 24} f_{4}^{\prime}-k_{f r 2} f_{S}^{\prime}\right)+M_{2}\left(f_{R 1} k_{p 12}+f_{R 3} k_{p 32}+f_{R 4} k_{p 42}+f_{R 5} k_{p 52}\right) \\
& 0=f_{R 3}\left(-k_{p 31} f_{1}^{\prime}{ }_{1}-k_{p 32} f_{2}^{\prime}{ }_{2}-k_{p 34} f_{4}^{\prime}{ }_{4}-k_{f r 3} f_{S}^{\prime}\right)+f^{\prime}{ }_{3}\left(f_{R 1} k_{p 13}+f_{R 2} k_{p 32}+f_{R 4} k_{p 43}+f_{R 5} k_{p 53}\right) \\
& 0=f_{R 4}\left(-k_{p 41} f_{1}^{\prime}{ }_{1}-k_{p 42} f_{2}^{\prime}{ }_{2}-k_{p 43} f_{3}^{\prime}{ }_{3}-k_{f r 4} Q_{1}\right)+D_{4}\left(f_{R 1} k_{p 14}+f_{R 2} k_{p 24}+f_{R 3} k_{p 34}+f_{R 5} k_{p 54}\right) \\
& 0=f_{R 5}\left(-k_{p 51} M_{1}-k_{p 52} M_{2}-k_{p 53} D_{3}-k_{p 54} D_{4}\right)+Q_{1}\left(f_{R 1} k_{f r 1}+f_{R 2} k_{f r 2}+f_{R 3} k_{f r 3}+f_{R 4} k_{f r 4}\right) \\
& 1=f_{R 1}+f_{R 2}+f_{R 3}+f_{R 4}+f_{R 5}
\end{aligned}
$$


Fractions $f_{i}$ of each radical are obtained by solving the system of linear equations (31) - (36). These fractions will be useful in the determination of pseudokinetic constants.

\subsection{Model Description}

\subsubsection{Mass Balance}

Besides the differential equation of the balances of initiator, monomer $A$ and monomer B, the model has a set of ordinary differential equations for the balances of radicals and dead chains of each size (population balance for the MWD).

The balances were formulated according to the reaction scheme (mechanism) shown in Table 1 that takes into account the classical steps of free radical polymerization in a batch reactor. (Figure 6).

Figure 6. Inverse suspension polymerization in a batch reactor

$A+B \rightarrow C$

A

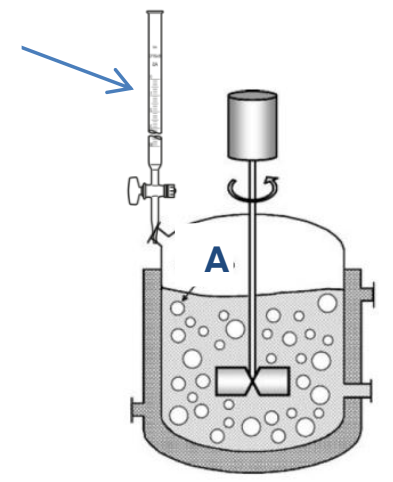

Ref.( Olivo 2015)

A : Organic Phase ( Toluene and Span 60)

B: Aquouse Phase ( Acrylic acid, tmpta, water and Sodium Persulfate) 


\section{Molar Balances of non-polymeric species}

The mole balance in the semi-batch reactor for the non-polymeric molecular species, for monomers and polymeric groups present have the following expressions:

Initiator

$\frac{d N_{I}}{d t}=\frac{d\left(V C_{I}\right)}{d t}=q_{e} C_{I_{e}}-K_{d} C_{I} V$

\section{Primary Radical}

$$
\frac{d N R_{0}}{d t}=\frac{d\left(V C_{R_{0}}\right)}{d t}=\left(2 k_{d} f[I]-\sum_{j=1}^{2} k_{l j}\left[R_{0}\right]\left[M_{j}\right]-\sum_{i=3}^{4} k_{l i}\left[R_{0}\right]\left[D_{i}\right]-k_{h}\left[R_{0}\right] Q_{1}\right) V
$$

\section{Acrylic acid $-\mathbf{M}_{1}$}

$$
\frac{d N M_{1}}{d t}=\frac{d\left(V C_{M_{1}}\right)}{d t}=q_{e} C_{M_{1}}+\left(-k_{I 1}\left[R_{0} \llbracket\left[M_{1}\right]-\sum_{i=1}^{5} \sum_{r=1}^{\infty} k_{p i l}\left[R_{r, i}\right]\left[M_{1}\right]\right) V\right.
$$

TMPTA-Trimethylolpropane triacrylate

$$
\frac{d N M_{2}}{d t}=\frac{d\left(V C_{M_{2}}\right)}{d t}=q_{e} C_{M_{2}}+\left(-k_{I 2}\left[R_{0}\right]\left[M_{2}\right]-\sum_{i=1}^{5} \sum_{r=1}^{\infty} k_{p i 2}\left[R_{r, i}\right]\left[M_{2}\right]\right) V
$$

Pendant double bonds $\left(D_{3}\right)$ :

$$
\begin{gathered}
\frac{d N D_{3}}{d t}=\left(k_{I 2}\left[R_{0}\right]\left[M_{2}\right]-\sum_{i=1}^{5} \sum_{r=1}^{\infty} k_{p i 2}\left[R_{r, i}\right]\left[M_{2}\right]-k_{I 3}\left[R_{0}\right]\left[D_{3}\right]\right. \\
\left.-\sum_{j=1}^{5} \sum_{r=1}^{\infty} k_{p j 3}\left[R_{r, j}\right]\left[D_{3}\right]-R C_{D_{3}}\right) V
\end{gathered}
$$

Pendant double bonds $\left(D_{4}\right)$ :

$$
\begin{gathered}
\frac{d N D_{4}}{d t}=\left(k_{I 3}\left[R_{0}\right]\left[D_{3}\right]-k_{I 4}\left[R_{0} \llbracket\left[D_{4}\right]-\sum_{j=1}^{5} \sum_{r=1}^{\infty} k_{p j 4}\left[R_{r, j}\right]\left[D_{4}\right]+\sum_{j=1}^{5} \sum_{r=1}^{\infty} k_{p j 3}\left[R_{r, j}\right]\left[D_{3}\right]\right.\right. \\
\left.-R C_{D_{4}}+R C_{D_{3}}\right) V
\end{gathered}
$$




\section{Balance of Radicals}

Radical 1

$$
\begin{aligned}
\frac{d N R_{1}}{d t}= & \left(k_{I 1}\left[R_{0}\right]\left[M_{1}\right]-k_{p 12}\left[R_{1}\right]\left[M_{2}\right]-\sum_{i=1}^{5} k_{p i 1}\left[R_{i}\right]\left[M_{1}\right]-k_{p 11}\left[R_{1}\right]\left[M_{1}\right]\right. \\
& -\sum_{i=3}^{4} k_{p 1 i}\left[R_{1} \rrbracket\left[D_{i}\right]-k_{f r 1} Q_{1}\left[R_{1}\right]-\sum_{j=1}^{5} k_{t}\left[R_{1}\right]\left[R_{j}\right] V\right.
\end{aligned}
$$

Radical 2

$$
\begin{aligned}
\frac{d N R_{2}}{d t} & =\left(k _ { I 2 } \left[R_{0} \rrbracket\left[M_{2}\right]-k_{p 21}\left[R_{2}\right]\left[M_{1}\right]+\sum_{i=1}^{5} k_{p i 2}\left[R_{i}\right]\left[M_{2}\right]-k_{p 22}\left[R_{2}\right]\left[M_{2}\right]\right.\right. \\
& -k_{p 2 i}\left[R_{2}\right] \sum_{i=3}^{4}\left[D_{i}\right]-k_{f r 2} Q_{1}\left[R_{2}\right]-\sum_{j=1}^{5} k_{t}\left[R_{2}\right]\left[R_{j}\right] V
\end{aligned}
$$

Radical 3

$$
\begin{aligned}
\frac{d N R_{3}}{d t}= & \left(\sum_{i=1}^{5} k_{p i 3}\left[R_{i} \rrbracket\left[D_{3}\right]-k_{p 33}\left[D_{3}\right] \llbracket R_{3}\right]-\left[R_{3}\right] \sum_{i=1}^{2} k_{p 3 i}\left[M_{i}\right]\right. \\
& +k_{13}\left[R_{0} \rrbracket\left[D_{3}\right]-k_{p 34}\left[R_{3} \rrbracket\left[D_{4}\right]-k_{f r 3}\left[R_{3}\right] Q_{1}-\sum_{j=1}^{5} k_{t}\left[R_{j}\right] R_{3}\right] V\right.
\end{aligned}
$$

Radical 4

$$
\begin{aligned}
\frac{d N R_{4}}{d t}= & \left(\sum_{i=1}^{5} k_{p i 4}\left[R_{i}\right]\left[D_{4}\right]-k_{p 44}\left[D_{4}\right]\left[R_{4}\right]-\left[R_{4}\right] \sum_{i=1}^{2} k_{p 4 i}\left[M_{i}\right]\right. \\
& -k_{I 4}\left[R_{0}\right]\left[D_{4}\right]-k_{p 43}\left[R_{4}\right]\left[D_{3}\right]-k_{f r 4}\left[R_{4}\right] Q_{1}-\sum_{j=1}^{5} k_{t}\left[R_{j}\right]\left[R_{4}\right] V
\end{aligned}
$$

Radical 5

$$
\begin{aligned}
\frac{d N R_{5}}{d t} & =\left(-\sum_{j=1}^{2} k_{p 5 j}\left[R_{5}\right]\left[M_{j}\right]-\sum_{i=1}^{5} k_{f r i}\left[R_{i}\right] Q_{1}-k_{f r 5}\left[R_{5}\right] Q_{1}+k_{h}\left[R_{0}\right] Q_{1}\right. \\
& -\sum_{j=1}^{5} k_{t}\left[R_{j}\right]\left[R_{5}\right] V
\end{aligned}
$$




\section{Balance of Polymer chains}

Balance of polymeric radicals of size $1(R f=1)$ :

$$
\begin{aligned}
& \frac{d N R_{r=1}}{d t}=\left(k _ { I } \left[R_{0} \rrbracket[M]-k_{p}\left[R_{r=1}\right][M]-k_{f r} s\left[P_{s}\right] \llbracket\left[R_{r=1}\right]-\sum_{s=1}^{\infty} k_{t}\left[R_{r=1} \rrbracket\left[R_{s}\right]\right.\right.\right. \\
& -k_{P D}\left[R_{r=1} \rrbracket\left[Q_{1}\right]+k_{I D}\left[R_{0}\right] Q_{1}\right) V
\end{aligned}
$$

Balance of polymeric radicals of size "r" (Rr):

$$
\begin{aligned}
\frac{d N R_{r}}{d t} & =\left(k_{p}\left[R_{r-1}\right][M]-k_{p}\left[R_{r}\right][M]+k_{I D}\left[R_{0}\right] r\left[P_{r}\right]-k_{P D}\left[R_{r}\right] Q_{1}-k_{f r}\left[R_{r}\right] Q_{1}\right. \\
& \left.+k_{f r} r\left[P_{r}\right] Y_{0}+k_{h} r\left[P_{r}\right] Y_{0}+k_{P D} \sum_{s=1}^{r-1} s\left[P_{s}\right]\left[R_{r-s}\right]-k_{t}\left[R_{r}\right] Y_{0}\right) V
\end{aligned}
$$

\section{Balance of dead polymer of size " $r$ " $(\mathrm{Pr})$}

$$
\begin{aligned}
\frac{d N P_{r}}{d t} & =\left(-k_{I D}\left[R_{0}\right] r\left[P_{r}\right]-k_{P D} Y_{0} r\left[P_{r}\right]-k_{f r} r\left[P_{r}\right] Y_{0}+k_{f r}\left[R_{r}\right] Q_{1}-k_{h} r\left[P_{r}\right]\left[R_{0}\right]\right. \\
& +\frac{k_{t c}}{2} \sum_{s=1}^{r-1}\left[R_{s} \llbracket\left[R_{r-s}\right]+k_{t d}\left[R_{r}\right] Y_{0}\right) V
\end{aligned}
$$

\section{Balance of Volume}

$$
\frac{d V}{d t}=q_{e}+\left(k_{I}\left[R_{0}\right][M]+k_{p} Y_{0}[M] V(72.06)\left(\frac{1}{\rho_{u}}-\frac{1}{\rho_{m}}\right)\right.
$$

where $72.06(\mathrm{~g} / \mathrm{mol})$ is the molar mass of the monomer, $\rho_{\mathrm{u}}=1150 \mathrm{~g} / \mathrm{L}$ is the polymer density and $\rho_{m}=1050 \mathrm{~g} / \mathrm{L}$ is the monomer density, and,

$\mathrm{k}_{1}$ : rate constant for initiation;

$\mathrm{R}_{0}$ : Concentration of primary radicals;

M: Concentration monomer;

$\mathrm{k}_{\mathrm{p}}$ : propagation rate constant;

$\mathrm{Y}_{0}$ : Moment of order zero for total radicals;

$\mathrm{V}$ : Volume of the reaction medium; 
$\rho_{\mathrm{U}}$ : Density of the monomer unit $(\mathrm{g} / \mathrm{L})$;

$\rho_{\mathrm{M}}$ : Density of the monomer $(\mathrm{g} / \mathrm{L})$.

$\mathrm{C}_{\mathrm{I}}$ : molar concentration to Sodium Persulfate (moles/liter)

$\mathrm{C}_{\mathrm{M} 1}$ : molar concentration to Acrylic acid (moles $\left./ \mathrm{ml}\right)$

$\mathrm{C}_{\mathrm{M} 2}$ : molar concentration to Trimethylolpropanetriacrylate (moles/liter)

$\mathrm{C}_{\mathrm{D} 3}$ : molar concentration to Pendant double bond (moles/liter)

$\mathrm{C}_{\mathrm{D} 4}$ : molar concentration to Pendant double bond (moles/liter)

$\mathrm{q}_{\mathrm{e}}$ : Feed Flow Rate (L/min)

\subsubsection{Balance of Moments}

As commonly found in several other polymerization systems, the analytical solution for the molecular weight distribution (MWD) is not possible for the system under study. Under these circumstances, techniques such as moment generating functions or the method of moments can be used. The method of moments can be employed to evaluate the first moments of the MWD, from which the molecular weight averages and polydispersity can be calculated (Aguiar et al., 2014; Souza, 2013).

The moment of order " $i$ " of the MWD of the polymeric radical ("live polymer", $\mathrm{Y}_{\mathrm{i}}$, and the moment of order " $\mathrm{i}$ " of the MWD of the polymer chains ("dead polymer"), $Q_{i}$, are defined by:

$$
Y_{i}=\sum_{r=1}^{\infty} r^{i} R_{r}
$$

$$
Q_{i}=\sum_{r=1}^{\infty} r^{i} P_{r}
$$


where $R_{r}$ represents the polymeric radical containing "r" monomeric units and $P_{r}$ represents the dead polymer containing "r" monomer units.

\section{Moment of order 0:}

The moment of order 0 (zero) for radicals has physical meaning, it is the total molar concentration of radicals in the reaction mixture contain all the possible sizes of the chain.

Applying the moment of zero order to equations (48) and (49), respectively:

$$
\begin{aligned}
& \sum_{R=1}^{1} r^{0} x \frac{1}{v} \frac{d N R_{1}}{d t}=k_{I}\left[R_{0}\right][M]-k_{p}[M] \sum_{r=1}^{1} r^{0} x\left[R_{1}\right]-k_{f r} Q_{1} \sum_{r=1}^{1} r^{0} x\left[R_{1}\right]- \\
& k_{t} Y_{0} \sum_{r=1}^{1} r^{0} x\left[R_{1}\right]-k_{P D} Q_{1} \sum_{r=1}^{1} r^{0} x\left[R_{1}\right]+k_{I D}\left[R_{0}\right] Q_{1} \\
& \sum_{R=2}^{\infty} r^{0} x \frac{1}{v} \frac{d N R_{r}}{d t}=k_{p}[M] \sum_{R=1}^{\infty}(r+1)^{0} x\left[R_{r}\right]-k_{p}[M] \sum_{R=2}^{\infty} r^{0} x\left[R_{r}\right]+k_{I D}\left[R_{0}\right] Q_{1}- \\
& k_{P D} \sum_{R=2}^{\infty} r^{0} x\left[R_{r}\right] Q_{1}-k_{f r} Q_{1} \sum_{R=r}^{\infty} r^{0} x\left[R_{r}\right]+k_{f r} Q_{1} Y_{0}+k_{h}\left[R_{0}\right] Q_{1}+ \\
& k_{P D} \sum_{s=1}^{r-1} s\left[P_{s} \llbracket\left[R_{r-s}\right]-k_{r} Y_{0} \sum_{R=2}^{\infty} r^{0}\left[R_{r}\right]\right.
\end{aligned}
$$

Adding equations (54) and (55), one gets:

$$
\begin{aligned}
\sum_{R=1}^{\infty} r^{0} \frac{1}{V} \frac{d N R_{r}}{d t} & =K_{I}\left[R_{0}\right][M]-K_{p}[M] \sum_{R=1}^{\infty} r^{0}\left[R_{r}\right]-K_{f r} Q_{1} \sum_{R=1}^{\infty} r^{0}\left[R_{r}\right] \\
& -K_{t} Y_{0} \sum_{R=1}^{\infty} r^{0}\left[R_{r}\right]-K_{P D} \sum_{R=1}^{\infty} r^{0}\left[R_{r}\right] Q_{1}+K_{I D}\left[R_{0}\right] Q_{1} \\
& +K_{p}[M] \sum_{R=1}^{\infty}(r+1)^{0}\left[R_{r}\right]+K_{I D}\left[R_{0}\right] Q_{1}+K_{f r} Q_{1} Y_{0} \\
& +K_{h} Q_{1}\left[R_{0}\right]+K_{P D} Y_{0} Q_{1} \\
\Leftrightarrow & \\
\frac{1}{V} \frac{d Y_{0}}{d t}=K_{I}\left[R_{0}\right][ & M]-K_{p}[M]\left[Y_{0}\right]-K_{f r} Q_{1} Y_{0}-K_{t} Y_{0} Y_{0} \\
& -K_{P D} Y_{0} Q_{1}+K_{I D}\left[R_{0}\right] Q_{1}+K_{p}[M] Y_{0}+K_{I D}\left[R_{0}\right] Q_{1} \\
& +K_{f r} Q_{1} Y_{0}+K_{h} Q_{1}\left[R_{0}\right]+K_{P D} Y_{0} Q_{1}
\end{aligned}
$$


Thus simplifying the above equation, we arrive at the following expression:

$$
\frac{1}{V} \frac{d Y_{0}}{d t}=K_{I}\left[R_{0} \llbracket[M]+2 K_{I D}\left[R_{0}\right] Q_{1}+K_{h} Q_{1}\left[R_{0}\right]-K_{t} Y_{0}^{2}\right.
$$

Applying the hypothesis pseudo- steady state (PSSH), we obtain:

$$
k_{I}\left[R_{0}\right][M]+2 k_{I D}\left[R_{0}\right] Q_{1}+k_{h} Q_{1}\left[R_{0}\right]-k_{t} Y_{0}^{2}=0
$$

This is an equation of second order to be solved using the Bhaskara formula (Souza, 2013).

\section{Moment of first order}

For the development of the balance equations of the moment of first order, the same reasoning used in the equations of moment of order 0 applies, differing only in the multiplication factor, which goes from $r_{0}$ to $r_{1}$. The final expression is deduced below:

$$
\begin{aligned}
& \frac{d Y_{1}}{d t}=k_{I}\left[R_{0}\right][M]+k_{p} Y_{0}[M]+k_{I D}\left[R_{0}\right] Q_{2}-k_{P D} Q_{1} Y_{1}-k_{f r} Q_{1} Y_{1}+k_{h} Q_{2}\left[R_{0}\right]+k_{f r} Q_{2} Y_{0}+ \\
& +k_{P D}\left(Q_{2} Y_{0}+Q_{1} Y_{1}\right)-k_{t} Y_{0} Y_{1} \\
& \begin{aligned}
\frac{1}{V} \frac{d Y_{1}}{d t}= & k_{I}\left[R_{0}\right][M]+k_{p} Y_{0}[M]+k_{I D}\left[R_{0}\right] Q_{2}-k_{P D} Q_{1} Y_{0}-k_{f r} Q_{1} Y_{1} \\
& +k_{h} Q_{2}\left[R_{0}\right]+k_{f r} Q_{2} Y_{0}+k_{P D}\left(Q_{2} Y_{0}+Q_{1} Y_{1}\right)+k_{t} Y_{0} Y_{1}
\end{aligned}
\end{aligned}
$$

Applying the case of the pseudo-steady state is obtained:

$$
Y_{1}=\frac{k_{I}\left[R_{0}\right][M]+k_{p} Y_{0}[M]+k_{I D}\left[R_{0}\right] Q_{2}+k_{h} Q_{2}\left[R_{0}\right]+k_{f r} Q_{2} Y_{0}+k_{P D} Q_{2} Y_{0}}{k_{f r} Q_{1}+k_{t} Y_{0}}
$$

\section{Overall moment for the dead Polymer}

Following the reasoning used to refer to the moments of the polymeric radicals, the following equations are obtained: 
Moment of zero order:

$$
\frac{1}{V} \frac{d Q_{0}}{d t}=-k_{I D}\left[R_{0}\right] Q_{1}-k_{P D} Y_{0} Q_{1}-k_{h} Q_{1}\left[R_{0}\right]+\frac{k_{t c}}{2} Y_{0}^{2}+k_{t d} Y_{0}^{2}
$$

Moment of first order

$$
\frac{1}{V} \frac{d Q_{1}}{d t}=-k_{I D}\left[R_{0}\right] Q_{2}-k_{P D} Y_{0} Q_{2}-k_{f r} Y_{0} Q_{2}+k_{f r} Y_{1} Q_{1}-k_{h} Q_{2}\left[R_{0}\right]+\frac{k_{t c}}{2}\left(2 Y_{0} Y_{1}\right)+k_{t d} Y_{0} Y_{1}
$$

Given this, it is necessary to eliminate the $Q_{2}$ equation model. To this end, it replaces $Y_{1}$ expression of the moment of order 1, and isolating $Q_{2}$ :

$$
\frac{1}{V} \frac{d Q_{1}}{d t}=-k_{I}\left[R_{0}\right][M]-k_{P} Y_{0}[M]
$$

\subsubsection{Numerical Fractionation Technique}

For polymerization systems that form gels (polymer network), the moments of order higher than 1 diverges (i.e., tends to infinite) at the gel point (the point at which the gel is formed). The technique of numerical fractionation was developed by Teymour and Campbell (1994) to deal with these problems. In this technique, the overall polymer population is divided numerically into a series of subdistributions, called "generations", having similar scale or degree of clustering and sizes, due to the process through which they were formed. As one moves from one generation to the next, the average molecular size will grow geometrically, thus leading (at least theoretically) toward a generation of infinitely large polymer molecules, which is the gel. At the beginning of the process, there are only linear chains, grouped as "Generation Zero". Then the first crosslinks are formed, e.g., when a primary radical reacts with a pendant double bond (PDB) of a linear chain, forming a branched chain ("First Generation") (Figure 7). Polymer chains of the First Generation can continue reacting with linear chains, still remaining in the First

Generation. The change to the "Second Generation" occurs when two chains of 
the First Generation react by a crosslinking or by termination by combination. The resulting molecule can continue to add linear chains and chains of the First Generation, still remaining in the Second Generation, until reaction with another chain of the Second Generation or a higher generation, thus moving to another generation. After a certain number of these transitions, the resulting polymer chain will be sufficiently large to be considered that the gel was formed (gel point) (Aguiar et al., 2014; Souza, 2013; Teymour and Campbell, 1994).

Figure 7. Numerical Fractionation Technique - transition of generation

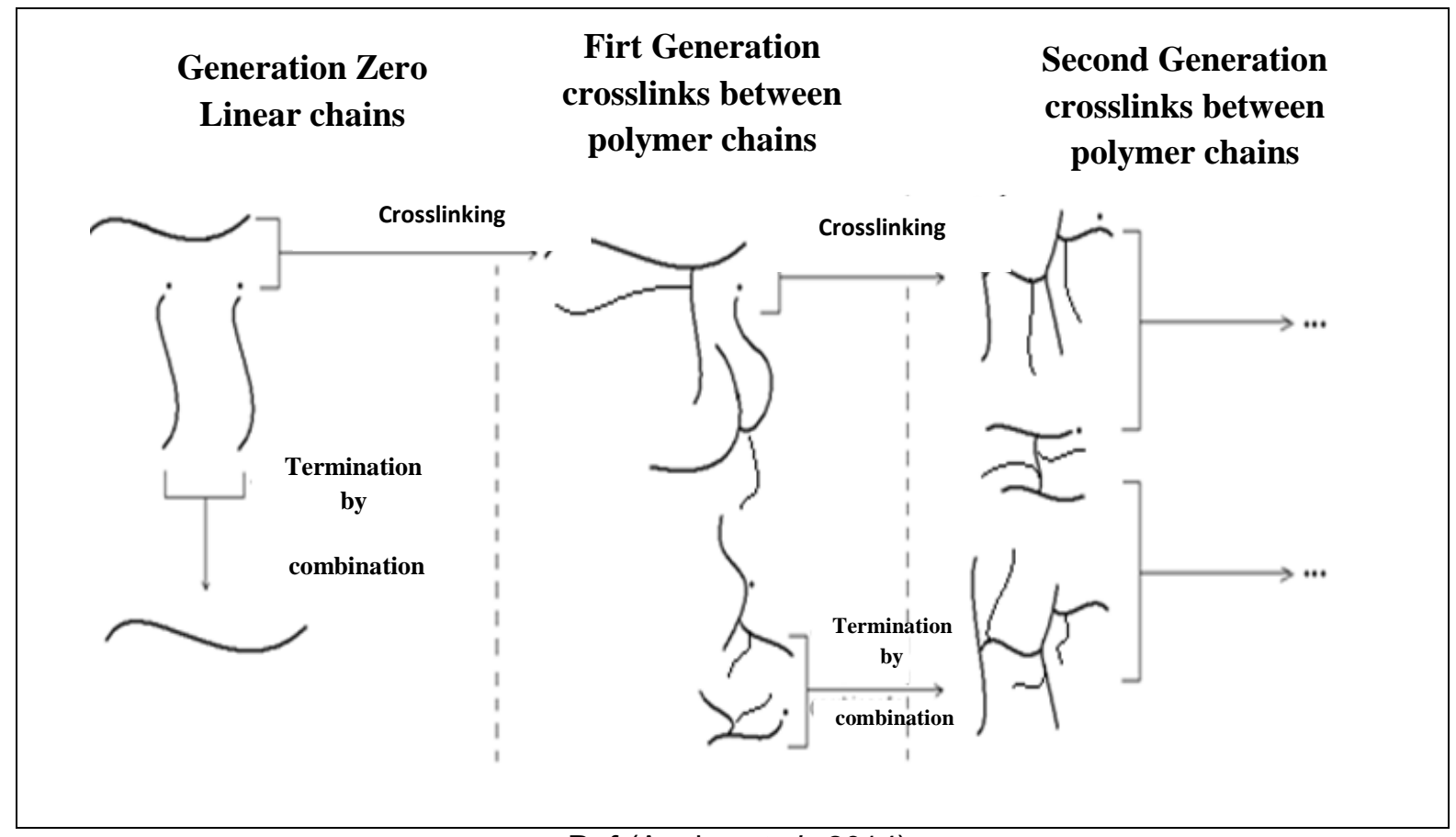

Ref (Aguiar et al., 2014)

The method of numerical fractionation is applied in this way: The maximum number of generations $\mathrm{N}$ of the sol polymer population is chosen ( $\mathrm{N}$ is a parameter of this technique). The balances for the zero-moment and first-moment of each generation $0,1,2,3, \ldots, \mathrm{N}$ is numerically solved, together with the balances of zero-moment and first-moment for the whole polymer population. The gel amount is evaluated, at each instant, as the difference between the first-moment of the whole polymer population and the sum of the first-moments of the $\mathrm{N}$ generations. 
The simulation is repeated for different number of generations $\mathrm{N}$ until the result does not change significantly and become independent of $\mathrm{N}$. Teymour and Campbell (1994) states that the use of 5 generations is usually enough to achieve this condition.

\section{Balance equations for the zero generation}

The generation zero contains only linear chains.

Polymeric radicals of zero generation

$$
\begin{aligned}
& Y_{00}=\frac{k_{I}\left[R_{0} \llbracket[M]\right.}{k_{f r} Q_{1}+k_{P D} Q_{1}+k_{t} Y_{0}} \\
& Y_{01}=\frac{k_{I}\left[R_{0}\right][M]+k_{p} Y_{00}[M]}{k_{f r} Q_{1}+k_{P D} Q_{1}+k_{t} Y_{0}} \\
& Y_{02}=\frac{k_{I}\left[R_{0} \llbracket[M]+k_{p}[M]\left(2 Y_{01}+Y_{00}\right)\right.}{k_{f r} Q_{1}+k_{P D} Q_{1}+k_{t} Y_{0}}
\end{aligned}
$$

Dead Polymer of the zero generation

\section{Zero Order}

$$
\begin{aligned}
\frac{d Q_{00}}{d t}= & -k_{I D}\left[R_{0}\right] Q_{01}-k_{P D} Y_{0} Q_{01}-k_{f r}\left(Q_{01} Y_{0}-Q_{1} Y_{00}\right)-k_{h} Q_{01}\left[R_{0}\right]+\frac{k_{t c}}{2} Y_{00}{ }^{2} \\
& +k_{t d} Y_{00} Y_{0}
\end{aligned}
$$

\section{First Order:}

$$
\begin{aligned}
\frac{d Q_{01}}{d t}= & -k_{I D}\left[R_{0}\right] Q_{02}-k_{P D} Y_{0} Q_{02}-k_{f r}\left(Q_{02} Y_{0}-Q_{1} Y_{01}\right)-k_{h} Q_{02}\left[R_{0}\right]+k_{t c} Y_{01} Y_{00}+ \\
& +k_{t d} Y_{01} Y_{0}
\end{aligned}
$$




\section{Second Order:}

$$
\begin{aligned}
\frac{d Q_{02}}{d t}= & -k_{I D}\left[R_{0}\right] Q_{03}-k_{P D} Y_{0} Q_{03}-k_{f r} Y_{0} Q_{03}+k_{f r} Q_{1} Y_{02}-k_{h} Q_{03}\left[R_{0}\right] \\
& +k_{t c}\left(Y_{02} Y_{00}+Y_{01}{ }^{2}\right)+k_{t d} Y_{02} Y_{0}
\end{aligned}
$$

\section{Balance equations for first generation}

Polymeric radicals of first generation

- Zero Order

$$
Y_{10}=\frac{k_{f r} Y_{0}\left(Q_{01}+Q_{11}\right)+k_{h}\left[R_{0}\right]\left(Q_{01}+Q_{11}\right)+k_{P D} Y_{00}\left(Q_{01}+Q_{11}\right)+k_{I D}\left[R_{0}\right]\left(Q_{00}+Q_{11}\right)}{k_{P D}\left(Q_{1}-Q_{01}\right)+k_{f r} Q_{1}+k_{t} Y_{0}}
$$

- First Order

$$
\begin{aligned}
& k_{p} Y_{10}[M]+k_{f r}\left(Y_{0} Q_{02}+Y_{0} Q_{12}\right)+k_{h}\left(\left[R_{0}\right] Q_{02}+\left[R_{0}\right] Q_{12}\right)+k_{I D}\left(\left[R_{0}\right] Q_{02}+\left[R_{0}\right] Q_{12}\right) \\
Y_{11}= & +k_{P D}\left(Q_{02} Y_{00}+Q_{01} Y_{01}+Q_{02} Y_{10}+Q_{12} Y_{00}+Q_{11} Y_{01}\right)
\end{aligned}
$$

- Second Order

$$
\begin{aligned}
& \begin{array}{l}
k_{p}\left(2 Y_{11}[M]+Y_{10}[M]\right)+k_{f r} Y_{0}\left(Q_{03}+Q_{13}\right)+k_{h}\left[R_{0}\right]\left(Q_{03}+Q_{13}\right)+k_{I D}\left[R_{0}\right]\left(Q_{03}+Q_{13}\right) \\
Y_{12}=
\end{array} \\
&+k_{P D}\left(Q_{03} Y_{00}+2 Q_{02} Y_{01}+Q_{01} Y_{02}+Q_{03} Y_{10}+2 Q_{02} Y_{11}+Q_{13} Y_{00}+2 Q_{12} Y_{01}+Q_{11} Y_{02}\right)
\end{aligned}
$$

\section{Death Polymer of first generation}

\section{Zero Order}

$$
\frac{d Q_{10}}{d t}=-k_{I D}\left[R_{0}\right] Q_{11}-k_{P D} Y_{0} Q_{11}-k_{f r}\left(Q_{11} Y_{0}-Q_{1} Y_{10}\right)-k_{h} Q_{11}\left[R_{0}\right]+k_{t c} Y_{00} Y_{11}+k_{t d} Y_{0} Y_{10}
$$

\section{Firts Order}

$$
\frac{d Q_{11}}{d t}=-k_{I D}\left[R_{0}\right] Q_{12}-k_{P D} Y_{0} Q_{12}-k_{f r}\left(Q_{12} Y_{0}-Q_{1} Y_{11}\right)-k_{h} Q_{12}\left[R_{0}\right]+k_{t c} Y_{11} Y_{00}+k_{t c} Y_{10} Y_{01}+k_{t d} Y_{11} Y_{0}
$$




\section{Second Order}

$$
\begin{aligned}
\frac{d Q_{12}}{d t}= & K_{I D}\left[R_{0}\right] Q_{13}-K_{P D} Y_{0} Q_{13}-K_{f r} Q_{13} Y_{0}-K_{f r} Q_{1} Y_{12}-K_{h} Q_{13}\left[R_{0}\right] \\
& +K_{t c}\left(Y_{02} Y_{10}+2 Y_{01} Y_{11}+Y_{00} Y_{12}\right)+K_{t d} Y_{12} Y_{0}
\end{aligned}
$$

\section{Balance equations for the generation " $\mathrm{i}$ "}

\section{Polymeric radicals of generation "i"}

\section{Zero Order}

$$
Y_{i 0}=\frac{k_{f r} Y_{0} Q_{i, 1}+k_{h}\left[R_{0}\right] Q_{i, 1}+k_{P D}\left(Q_{i-1,1} Y_{i-1,0}+Q_{i, 1} \sum_{j=0}^{i-1} Y_{j, 0}\right)+k_{I D}\left[R_{0}\right] Q_{i, 1}}{k_{P D}\left(Q_{1}-\sum_{j=0}^{i-1} Q_{j, 1}\right)+k_{f r} Q_{1}+k_{t} Y_{0}}
$$

First Order

$$
\begin{aligned}
& \begin{array}{l}
k_{p} Y_{i, 0}[M]+k_{f r} Y_{0} Q_{i, 2}+k_{h}\left[R_{0}\right] Q_{i, 2}+k_{I D}\left[R_{0}\right] Q_{i, 2} \\
Y_{i 1}=
\end{array}
\end{aligned}
$$

\section{Second Order}

$$
\begin{gathered}
k_{p}[M]\left(2 Y_{i, 1}+Y_{i, 0}\right)+k_{f r} Y_{0} Q_{i, 3}+k_{h}\left[R_{0}\right] Q_{i, 3}+k_{I D}\left[R_{0}\right] Q_{i, 3} \\
+k_{P D}\left(\begin{array}{l}
2 Q_{i-1,3} Y_{i-1,0}+2 Q_{i-1,2} Y_{i-1,1}+Y_{i, 0} \sum_{j=0}^{i-1} Q_{j, 3}+Q_{i-1,1} Y_{i-1,2} \\
+2 Y_{i, 1} \sum_{j=0}^{i-1} Q_{j, 2}+Q_{i, 3} \sum_{j=0}^{i-1} Y_{j, 0}+2 Q_{i, 2} \sum_{j=0}^{i-1} Y_{j, 1}
\end{array}\right) \\
K_{P D}\left(Q_{1}-2 \sum_{j=0}^{i-1} Q_{j, 1}\right)+k_{f r} Q_{1}+k_{t} Y_{0}
\end{gathered}
$$

\section{Death Polymer of generation "i"}

\section{Zero Order}

$$
\begin{aligned}
\frac{d Q_{i 0}}{d t}= & -k_{I D}\left[R_{0}\right] Q_{i 1}-K_{P D} Y_{0} Q_{i 1}-k_{f r}\left(Q_{i 1} Y_{0}-Q_{1} Y_{i 0}\right)-k_{h} Q_{i 1}\left[R_{0}\right] \\
& +k_{t c}\left(\frac{Y_{i-1,0}^{2}}{2}+Y_{i 0} \sum_{j=0}^{i-1} Y_{j o}\right)+k_{t d} Y_{0} Y_{i 0}
\end{aligned}
$$




\section{First Order}

$$
\begin{aligned}
\frac{d Q_{i 1}}{d t}= & -k_{I D}\left[R_{0}\right] Q_{i 2}-k_{P D} Y_{0} Q_{i 2}-k_{f r}\left(Q_{i 2} Y_{0}-Q_{1} Y_{i 1}\right)-k_{h} Q_{i 2}\left[R_{0}\right] \\
& +k_{t c}\left(Y_{i-1.0} Y_{i-1.1}+Y_{i 0} \sum_{j=0}^{i-1} Y_{j 1}+Y_{i 1} \sum_{j=0}^{i-1} Y_{j 0}\right)+k_{t d} Y_{i 1} Y_{0}
\end{aligned}
$$

\section{Second Order}

$$
\begin{aligned}
\frac{d Q_{i 2}}{d t}= & -k_{I D}\left[R_{0}\right] Q_{i 3}-k_{P D} Y_{0} Q_{i 3}-k_{f r}\left(Q_{i 3} Y_{0}-Q_{1} Y_{i 2}\right)-k_{h} Q_{i 3}\left[R_{0}\right]+k_{t d} Y_{0} Y_{i 2} \\
& +k_{t c}\left(Y_{i-1.0}+Y_{i-1,2}+\left(Y_{i-1,0}\right)^{2}+Y_{i 0} \sum_{j=0}^{i-1} Y_{j 2}+2 Y_{i 1} \sum_{j=0}^{i-1} Y_{j 1}+Y_{i 2} \sum_{j=0}^{i-1} Y_{j 0}\right)
\end{aligned}
$$

Application of closure relation of Saidel and Katz (1968);[Aguiar., 2014]; [Souza, 2013]:

$Q_{i 3}=\frac{2 Q_{i 2}{ }^{2}}{Q_{i 1}}-\frac{Q_{i 2} Q_{i 1}}{Q_{i 0}}$ 


\section{CHAPTER IV}

\section{EXPERIMENTAL SECTION}

In this chapter, the materials and equipments used are described and the experimental procedures are explained.

\subsection{Materials}

Acrylic acid (AA) (Sigma-Aldrich, 99\% purity, containing 200 ppm of MEHQ as inhibitor) was used as the main water-soluble monomer. Trimethylolpropane triacrylate (TMPTA) (Sigma Aldrich, containing $600 \mathrm{ppm}$ of MEHQ)) and tetraallyloxyethane (TAO) were used as crosslinkers (multifunctional monomers). Sodium persulfate (Sigma-Aldrich) was employed as initiator. Toluene (Quimica Moderna) was chosen as the inert continuous phase in the inverse-suspension polymerizations, and Span 60 (Sigma Aldrich) as stabilizer. Hydroquinone (SigmaAldrich) was used as inhibitor to shortstop the polymerization reaction in the samples taken from the reactor. Unless otherwise stated, the reagents were used as received, without further purification.

Tables 3 and 4 present the formulation of the hydrogels prepared by the homopolymerization and by the copolymerization process, for the different studies. The effects of five experimental variables were investigated: temperature, initiator concentration, feed rate, crosslinker agent concentration and monomer concentration.

In Runs E1 to E14 (Table 3), monomer conversion was measured as a function of time. In Runs E5, E10 and E14, in addition to the monomer conversion, the gel fraction was also measured along the experiments.

In all experiments in Table 3, the reaction medium was continuously sparged with nitrogen to deoxygenate the mixture during the whole duration of the run, in order to reduce the effect of the inhibition by the dissolved oxygen in water. 
Table 3. Experimental Conditions

\begin{tabular}{|l|c|c|c|c|c|c|c|c|c|c|}
\hline & & & \multicolumn{6}{|c|}{ Formulation } & \\
\hline Run & $\mathrm{T}$ & Toluene & Span & Water & Acrylic & TMPTA & EGDMA \\
$\left({ }^{\circ} \mathrm{C}\right)$ & $(\mathrm{g})$ & $\begin{array}{c}\text { TAO } \\
(\mathrm{g})\end{array}$ & $\begin{array}{c}\text { Acid } \\
(\mathrm{g})\end{array}$ & $(\mathrm{g})$ & $\begin{array}{c}\text { Sodium } \\
\text { Persulfate } \\
(\mathrm{g})\end{array}$ & $\begin{array}{c}\text { Feeding } \\
\text { Time } \\
(\mathrm{min})\end{array}$ \\
\hline E1 & 70 & 191.18 & 3.54 & 30.8870 & 7.8443 & 0.1016 & 0 & 0 & 0.1512 & 40 \\
\hline E2 & 60 & 191.25 & 3.88 & 35.2797 & 7.7132 & 0.1081 & 0 & 0 & 0.15 & 40 \\
\hline E3 & 50 & 192.8 & 3.58 & 29.7509 & 7.714 & 0.1208 & 0 & 0 & 0.1522 & 40 \\
\hline E4 & 60 & 192.85 & 3.51 & 30.7055 & 7.713 & 0 & 0 & 0.1 & 0.1598 & 40 \\
\hline E5 & 60 & 196.12 & 3.56 & 34.5494 & 7.717 & 0 & 0 & 0.057 & 0.1569 & 40 \\
\hline E6 & 60 & 191.8 & 3.68 & 29.9852 & 7.68 & 0.1015 & 0 & 0 & 0.0689 & 40 \\
\hline E7 & 60 & 190.85 & 3.6 & 30.4854 & 7.8196 & 0.117 & 0 & 0 & 0.07581 & 40 \\
\hline E8 & 60 & 190.72 & 3.8 & 31.1329 & 7.8759 & 0.1175 & 0 & 0 & 0.1546 & 60 \\
\hline E9 & 60 & 190.99 & 3.61 & 26.3136 & 3.5363 & 0.10 & 0 & 0 & 0.1546 & 40 \\
\hline E10 & 60 & 191.75 & 3.6 & 30.2885 & 7.7702 & 0.05 & 0 & 0 & 0.1199 & 40 \\
\hline E11 & 60 & 193.47 & 3.59 & 30.0425 & 7.844 & 0.14 & 0 & 0 & 0.11 & 40 \\
\hline E12 & 60 & 191.4 & 4.0 & 30.1472 & 7.7218 & 0 & 0 & 0 & 0.2088 & 40 \\
\hline E13 & 60 & 646.5 & 12.09 & 141.76 & 26.2791 & 0 & 0 & 0 & 0.869 & 40 \\
\hline E14 & 60 & 193.78 & 3.72 & 30.4835 & 7.7866 & 0 & 0.0633 & 0 & 0.1976 & 40 \\
\hline
\end{tabular}

Experiments E15 to E18 in Table 4 were carried out under conditions similar to E6, except that the time of bubbling nitrogen in the reaction medium was varied to assess the possible inhibition effect of the dissolved oxygen. In these experiments, the monomer was previously purified by removing the inhibitor, as will be explained below. 
Table 4. Experimental conditions for the runs of the study of Inhibition period.

\begin{tabular}{|c|c|c|c|c|c|c|c|c|c|}
\hline \multicolumn{10}{|c|}{ Inhibition Period } \\
\hline Run & $\begin{array}{c}\text { T } \\
{ }^{\circ} \mathrm{C}\end{array}$ & $\begin{array}{c}\text { Toluene } \\
(\mathrm{g})\end{array}$ & $\begin{array}{c}\text { Span } \\
60 \\
(\mathrm{~g})\end{array}$ & $\begin{array}{c}\text { Water } \\
(\mathrm{g})\end{array}$ & $\begin{array}{c}\text { Acrylic } \\
\text { Acid } \\
(\mathrm{g})\end{array}$ & $\begin{array}{c}\text { TMPTA } \\
(\mathrm{g})\end{array}$ & $\begin{array}{c}\text { Induction } \\
\text { Period } \\
(\mathrm{min})\end{array}$ & $\begin{array}{c}\text { Sodium } \\
\text { Persulfate } \\
(\mathrm{g})\end{array}$ & $\begin{array}{c}\text { Feeding } \\
\text { Time } \\
(\mathrm{min})\end{array}$ \\
\hline E15 & 60 & 191.8 & 3.68 & 29.9852 & 7.68 & 0.1015 & 0 & 0.0689 & 40 \\
\hline E16 & 60 & 191.8 & 3.68 & 29.9852 & 7.68 & 0.1015 & 15 & 0.0689 & 40 \\
\hline E17 & 60 & 191.8 & 3.68 & 29.9852 & 7.68 & 0.1015 & 30 & 0.0689 & 40 \\
\hline E18 & 60 & 191.8 & 3.68 & 29.9852 & 7.68 & 0.1015 & 60 & 0.0689 & 40 \\
\hline
\end{tabular}

\subsection{Equipments}

The inverse suspension polymerizations were carried out in a a $250 \mathrm{~mL}$ fivenecked jacketed glass reactor, equipped with an impeller, reflux condenser, a dropping tube (buret), a thermometer, and nitrogen line. The reactor is shown in Figure 8. Water from a thermostatic bath circulated through the reactor jacket to control the temperature and discharge valve. 
Figure 8 Schematic of the reactor used in the inverse suspension polymerization reactions.

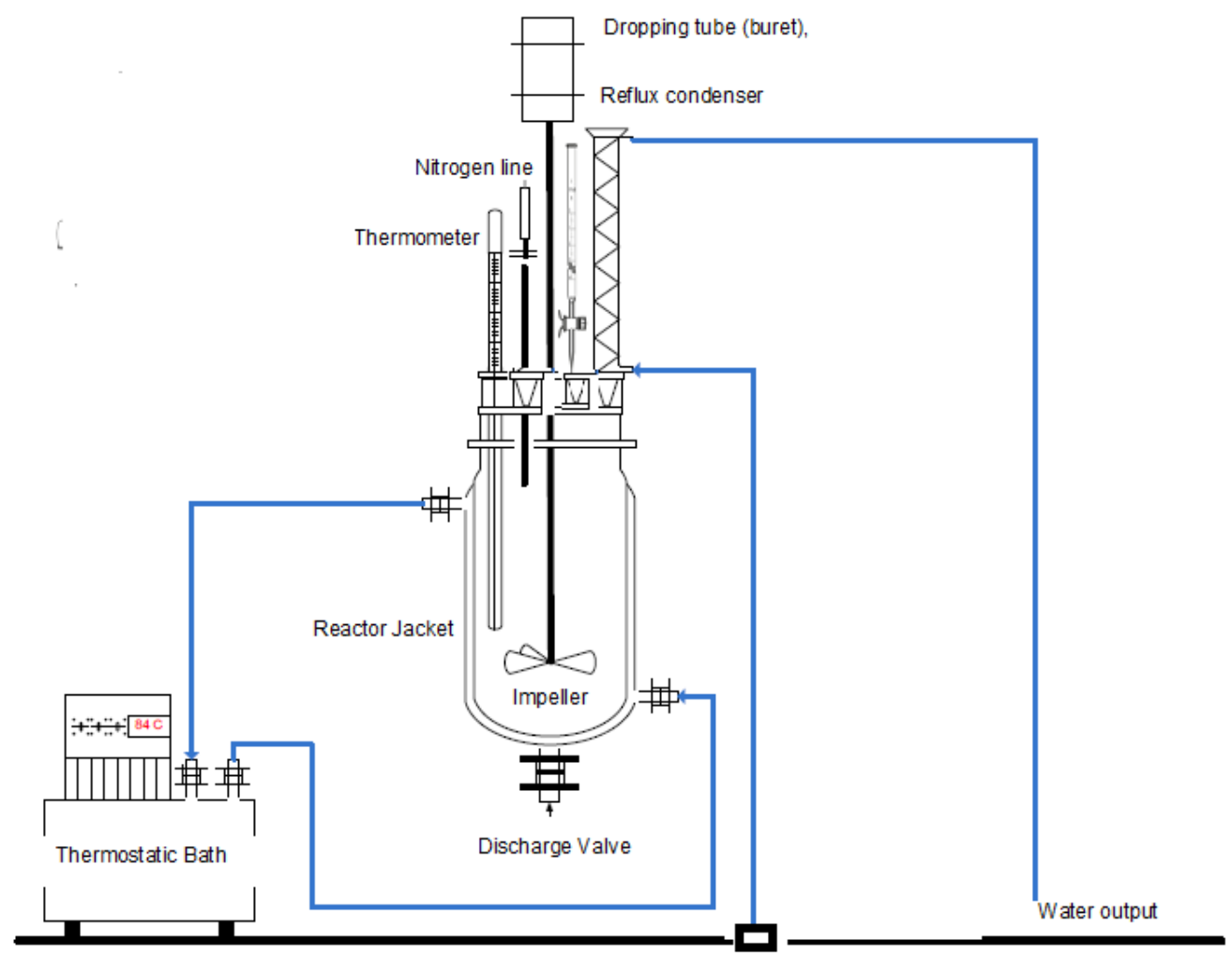

\subsection{Experimental Procedure:}

\subsubsection{Preparation of the organic phase}

The stabilizer Span 60 was dissolved in toluene at room temperature under magnetic stirring by 20 minutes. This solution was then transferred to the reactor.

\subsubsection{Preparation of the aqueous phase}

The aqueous solution was prepared by mixing water, acrylic acid, crosslinker TMPTA at room temperature using a magnetic stirrer by 10 minutes. 
Then the initiator (sodium persulfate) dissolved in a small amount of water was added to this mixture at room temperature.

\subsubsection{Polymerization runs}

The prepared organic solution (Span 60 and toluene) was transferred a 250 $\mathrm{mL}$ four-necked jacketed glass reactor. Water from a thermostatic bath flows through the jacket to control the temperature. The reactor content was heated (under a mild stream of nitrogen bubbling to remove dissolved oxygen from the medium) until reached the desired temperature.

Then the aqueous solution, composed of acrylic acid, crosslinker (when used), sodium persulfate and water, transferred to the buret, was added dropwise to the organic phase under an agitation speed of $400 \mathrm{rpm}$. The duration of the feeding was 40-60 min. At the end of the aqueous phase feeding period, the first sample was withdrawn from the reactor and the time was set to zero.

After finishing the feeding of the aqueous solution, samples were taken at different times along the operation time for the analysis of monomer conversion and gel content.

\subsubsection{Purification monomer}

Commercial monomers have some amount of inhibitor MEHQ to prevent spontaneous polymerization in the storage flask. Inhibitors and retarders are characterized by having the ability to form a stabilized free radical. The standard inhibitor level in commercial acrylic acid (Sigma-Aldrich) is 200 ppm Monomethyl Ether of Hydroquinone (MEHQ), and in commercial TMPTA (Sigma-Aldrich) is 600 ppm.

Experiments E6 and E15-E18 were performed with monomer (and crosslinker) without inhibitor MEHQ (Mono Methyl Ether of Hydroquinone). 
Monomers can be purified in lab scale by steam or vacuum distillation. In the present study, vacuum distillation was used (Figure 9). During the distillation, a copper gauze was placed in the monomer to inhibit polymerization.

Following the safety recommendations, after distillation, acrylic acid and TMPTA should be stored at a temperature range of 18 to $25^{\circ} \mathrm{C}$, to prevent any crystallization (freezing point $=13^{\circ} \mathrm{C}$ ). It is recommended that the monomers be stored indoors at a temperature lower than $25^{\circ} \mathrm{C}$ and away from light, under air atmosphere (because oxygen acts also as an inhibitor).

Figure 9. Vacuum Distillation equipment used for removing the MEHQ inhibitor.
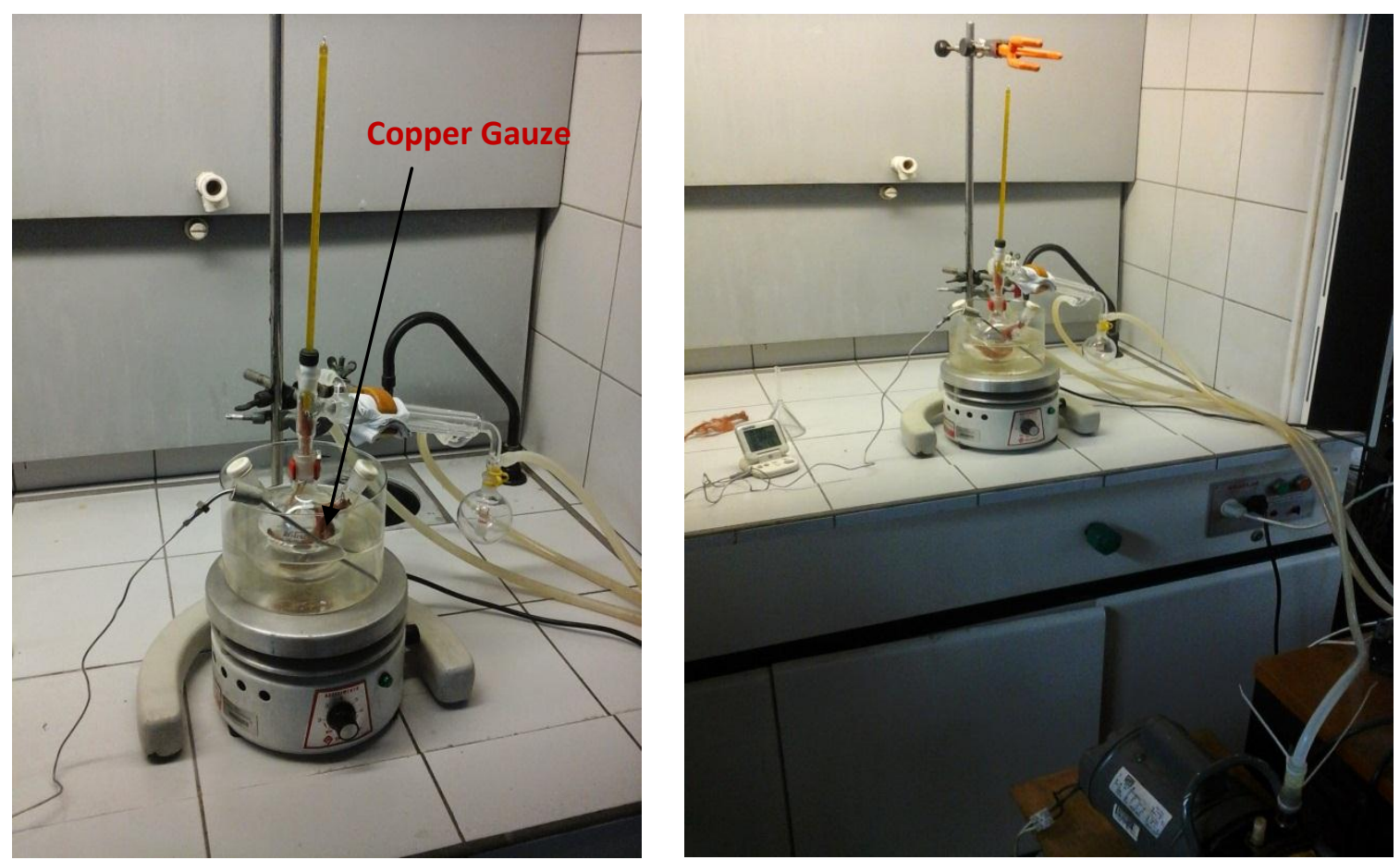

\subsubsection{Study of the inhibition period}

The inhibition period, usually observed in polymerization of commercial monomers, is the initial period of process in which no formation of polymer (no consumption of monomer) takes place from the starting of the polymerization 
process. This occurs due to the presence of inhibitor. Besides to inhibitor present in commercial grade monomers, oxygen is also a very active inhibitor for most free radical polymerization processes. Moreover, the solubility of oxygen in water is sufficiently high.

In order to study the effect of the oxygen as an inhibitor, the procedure proposed by Cutié et al. (1996) and Li et al. (2006) was employed in the present work. After removed the commercial MEHQ inhibitor from the monomer, it was aerated with air for $60 \mathrm{~min}$ to allow the saturation of the solution with oxygen. Then, the solution was deoxygenated at different levels by bubbling with nitrogen for 15 , 30, and 60 min (as indicated in Table 4). Monomer deoxygenated at different levels was employed in the polymerization runs (E15-E18) to test the effect of different oxygen concentrations on the monomer conversion. These runs were conducted with low concentration of initiator $(0.03 \% \mathrm{wt})$ in order to maximize the effect under study.

\subsubsection{Gravimetric analysis for conversion}

The monomer conversion during the polymerization was determined by gravimetric analysis, using analytical balance (with accuracy of $0.0001 \mathrm{~g}$ ). An empty glass container (Beaker) is previously weighed $\left(\mathrm{m}_{1}\right)$ and then an amount ( $0.25 \mathrm{~mL}$ ) of aqueous solution of $2 \%$ wt hydroquinone (inhibitor) was added (to shortstop the reaction), and the flask is weighed $\left(\mathrm{m}_{2}\right)$. Then, a sample of $\sim 10 \mathrm{~mL}$ taken from the reactor is poured into the flask and weighed again $\left(m_{3}\right)$. Samples were kept immersed in an ice bath, Figure 10, to prevent further polymerization. At the end of the experimental run, all samples were placed in an oven at $68^{\circ} \mathrm{C}$ for 48 hours in order to remove the volatile components (toluene and unreacted monomer). Then the samples were weighed again several times over a period of 4 days to ensure that the dry sample reached a constant weight $\left(m_{4}\right)$. Figure 11 illustrates the wet and dry samples. 
Figure 10. Samples immersed in an ice bath

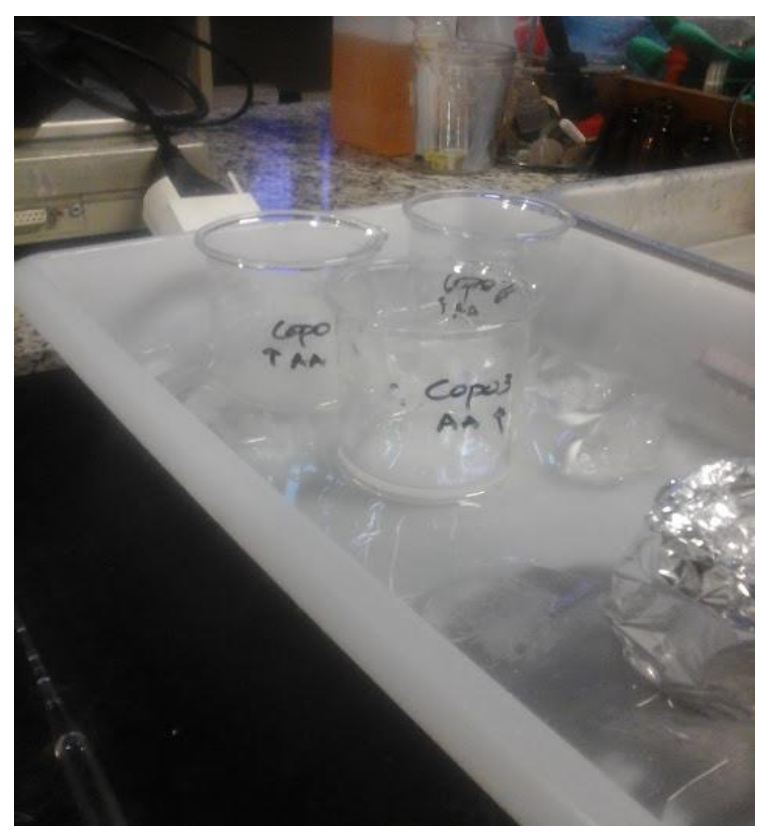

Figure 11. Dry samples

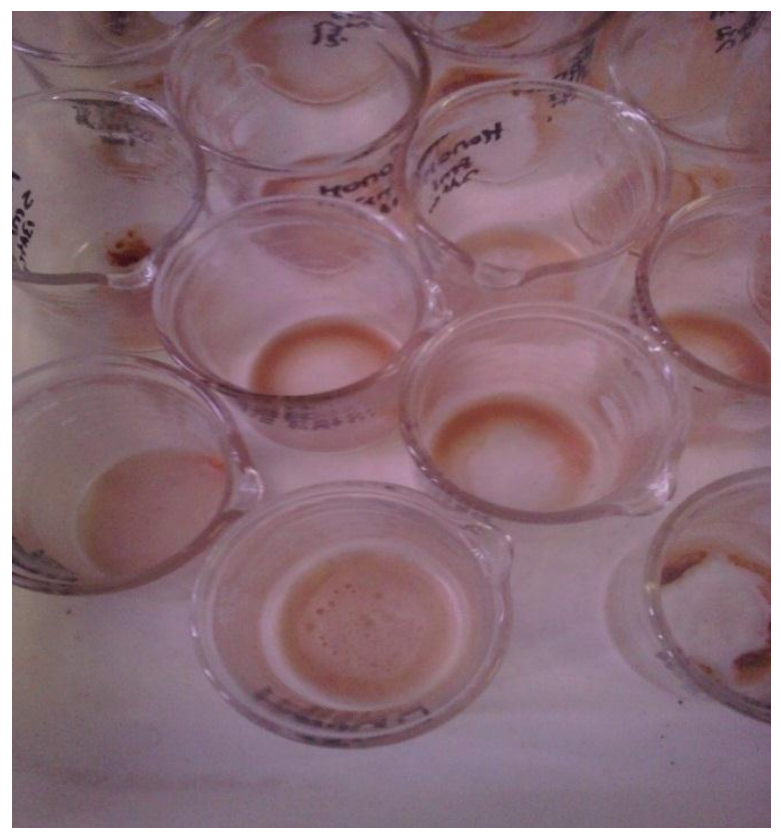

The conversion was calculated by equation (83):

$$
X=\frac{\left(m_{4}-m_{1}\right)-\left\lfloor\left(m_{3}-m_{2}\right) * f_{\text {span60 }}\right\rfloor-\left\lfloor\left(m_{2}-m_{1}\right) * f_{\text {hydoquinou }}\right\rfloor}{\left(m_{3}-m_{2}\right) * f_{\text {acrylicaaid }}}
$$

where

$\mathrm{m}_{1}=$ weight of the empty Beaker flask (g),

$\mathrm{m}_{2}=$ weight of the flask with hydroquinone solution $(\mathrm{g})$

$m_{3}=$ weight of the flask with the sample taken from the reactor plus $\mathrm{HQ}(\mathrm{g})$,

$\mathrm{m}_{4}=$ weight of the flask with the dry sample $(\mathrm{g})$,

$f_{\text {hydroquinone }}=$ weight fraction of the hydroquinone solution added to the sample $f_{\text {span60 }}=$ weight fraction of Span 60 in the formulation, $f_{\text {acrylic acid }}=$ weight fraction of acrylic acid in the formulation. 


\subsubsection{Determination of gel fraction by Soxhlet system}

The gel fraction of polymer samples was determined by extracting the sol fraction (soluble fraction) using a Soxhlet-type equipment (Soxtherm Gerhardt Multistat/SX PC), composed of 6 extraction units, multistate control unit, extraction glass beakers, filter paper, compressed air supply and thermal bath equipment, shown in Figure 12 (http://soxtherm.gerhardt.de/en/).

Figure 12. Soxhlet extraction equipment
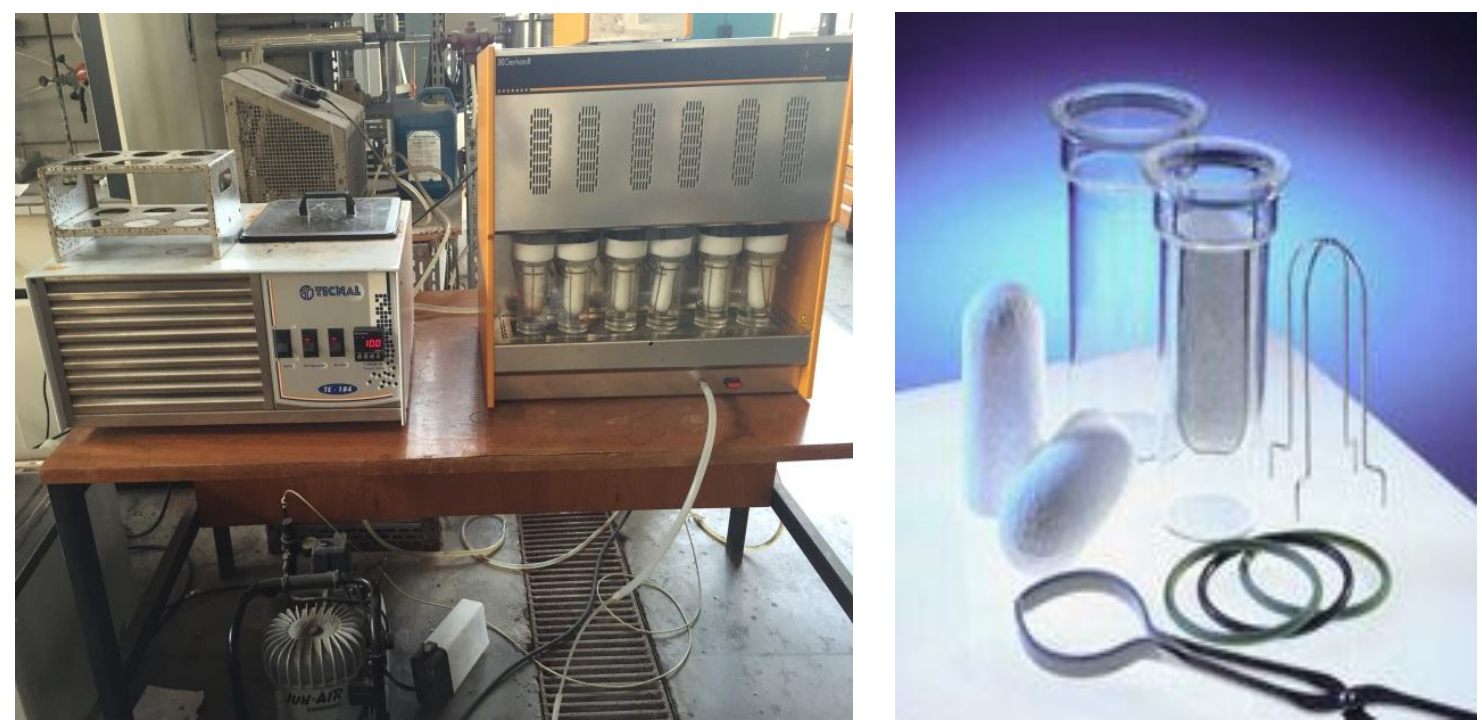

A sample of dry polymer was placed inside the thick filter paper (previously weighed, $\left.m_{5}\right)$ and weighed $\left(m_{6}\right)$. The filter paper with the polymer sample was placed in the extraction thimble, which was then loaded into the main chamber of the Soxtherm extractor. The extraction solvent (water, in the present case) was placed in the distillation flask and heated to reflux for 3 hours for the extraction of the soluble fraction of polymer (sol fraction). The filter paper with the insoluble polymer was then dried in an oven for about 24 hours, left in a desiccator for 2 hours for cooling down to the ambient temperature and then weighed $\left(m_{7}\right)$. 
The following equation was used to determinate the gel fraction:

$$
G F=\frac{\left(m_{7}-m_{5}\right)}{\left(m_{6}-m_{5}\right)}
$$

where

$m_{5}=$ weight of the empty filter paper $(\mathrm{g})$,

$m_{6}=$ weight of the initial dry polymer sample and the filter paper $(\mathrm{g})$,

$m_{7}=$ weight of the final dry sample after extraction and filter paper $(g)$. 


\section{CHAPTER V}

\section{RESULTS AND DISCUSSIONS}

In this Chapter, the experimental results of monomer conversion and gel fraction measured in the different experiments are presented and discussed. The results are also compared with the prediction of the mathematical model, and the sensitivity of the certain key parameters is tested.

The runs were performed typically, as a base case, in a total of four hours in batch operation, after $40 \mathrm{~min}$ of feeding period before the time zero (time was set to zero at the end of the feeding period), at temperature of $60^{\circ} \mathrm{C}$ and stirring speed of $400 \mathrm{rpm}$. Runs with changes in these conditions were carried out to assess the effects of the process conditions on the process performance.

\subsection{Conversion measured by Gravimetry}

\subsubsection{Homopolymerization}

Figure 13 presents the evolution of the monomer conversion measured during the homopolymerization of acrylic acid in run E12, in which no multifunctional monomer (crosslinker) was used.

The monomer conversion increased very slowly during the first 40 minutes (probably an effect of retardation/inhibition), and then increased fast, reaching conversion of almost $80 \%$ at $160 \mathrm{~min}$. The last two points, measured at conversions above $60 \%$, seems to be higher than the trend, probably an indication of autoacceleration effect (gel effect). 


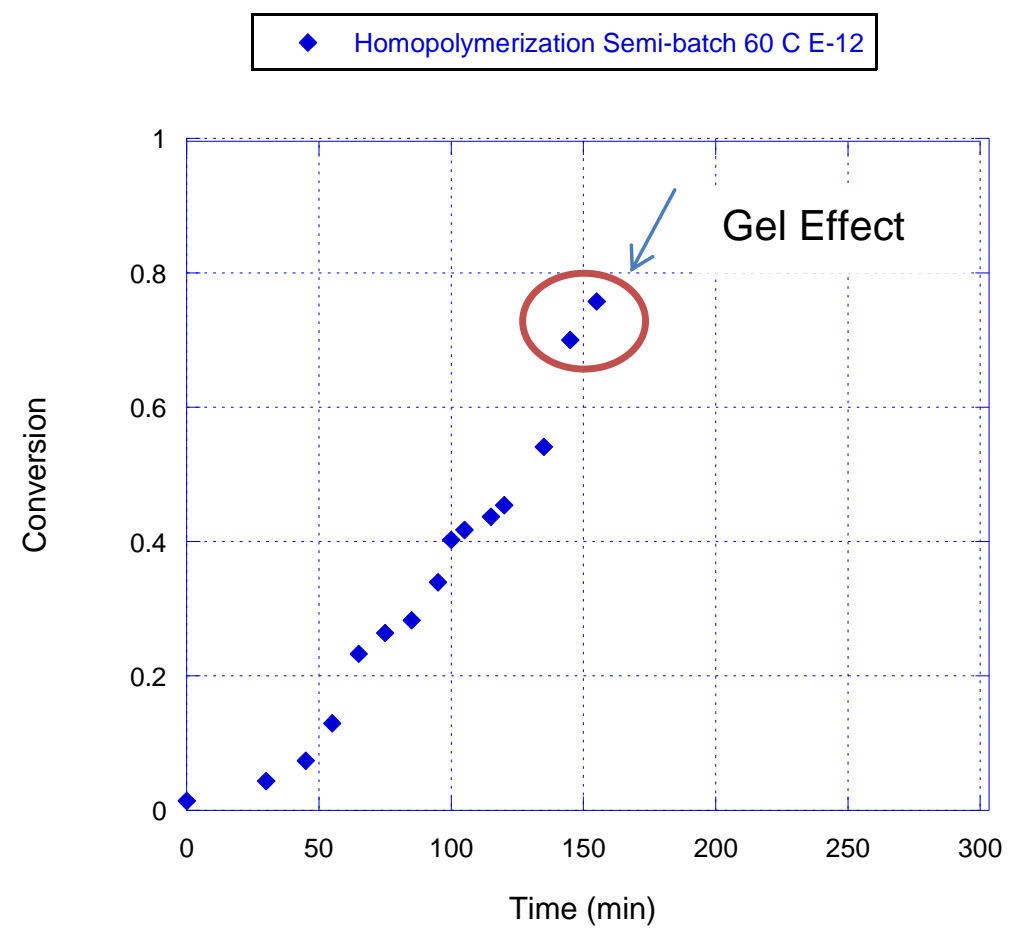

Figure 13. Conversion-time homopolymerization of acrylic acid in run E12.

\subsubsection{Reproducibility of conversion measurements}

Run E13 was carried out in a large reactor $(1 \mathrm{~L})$ in order to assess the reproducibility of the measurements of conversion. During this experiment, at each sampling time, two samples of the reaction medium were taken from the reactor and independently analyzed. Therefore, these results allows to assess the reproducibility of the measurements of conversion; however, not the reproducibility of the runs (because the samples were taken from the same experiment). The results are presented in Figure 14. The measurements are reproducible, but the differences can be, for some points, as large as $\pm 15 \%$. These relatively large experimental errors can be related to the size of the samples (if too small, the weighting measurement errors can become relatively large) and difficulties 
involved in complete drying of the sample due to formation of dry skin on the film surface.

\begin{tabular}{|l|c|c|c|c|c|c|c|c|c|c|}
\hline Experiments & $\begin{array}{c}\mathrm{T} \\
\left({ }^{\circ} \mathrm{C}\right)\end{array}$ & $\begin{array}{c}\text { Toluene } \\
(\mathrm{g})\end{array}$ & $\begin{array}{c}\text { Span } \\
60 \\
(\mathrm{~g})\end{array}$ & $\begin{array}{c}\text { Water } \\
(\mathrm{g})\end{array}$ & $\begin{array}{c}\text { Acrylic } \\
\text { Acid } \\
(\mathrm{g})\end{array}$ & $\begin{array}{c}\text { TMPTA } \\
(\mathrm{g})\end{array}$ & $\begin{array}{c}\text { EGDMA } \\
(\mathrm{g})\end{array}$ & $\begin{array}{c}\text { TAO } \\
(\mathrm{g})\end{array}$ & $\begin{array}{c}\text { Sodium } \\
\text { Persulfate } \\
(\mathrm{g})\end{array}$ & $\begin{array}{c}\text { Feeding } \\
\text { Time } \\
(\mathrm{min})\end{array}$ \\
\hline $\mathrm{E} 13$ & 60 & 646.5 & 12.09 & 141.76 & 26.2791 & 0 & 0 & 0 & 0.869 & 40 \\
\hline
\end{tabular}

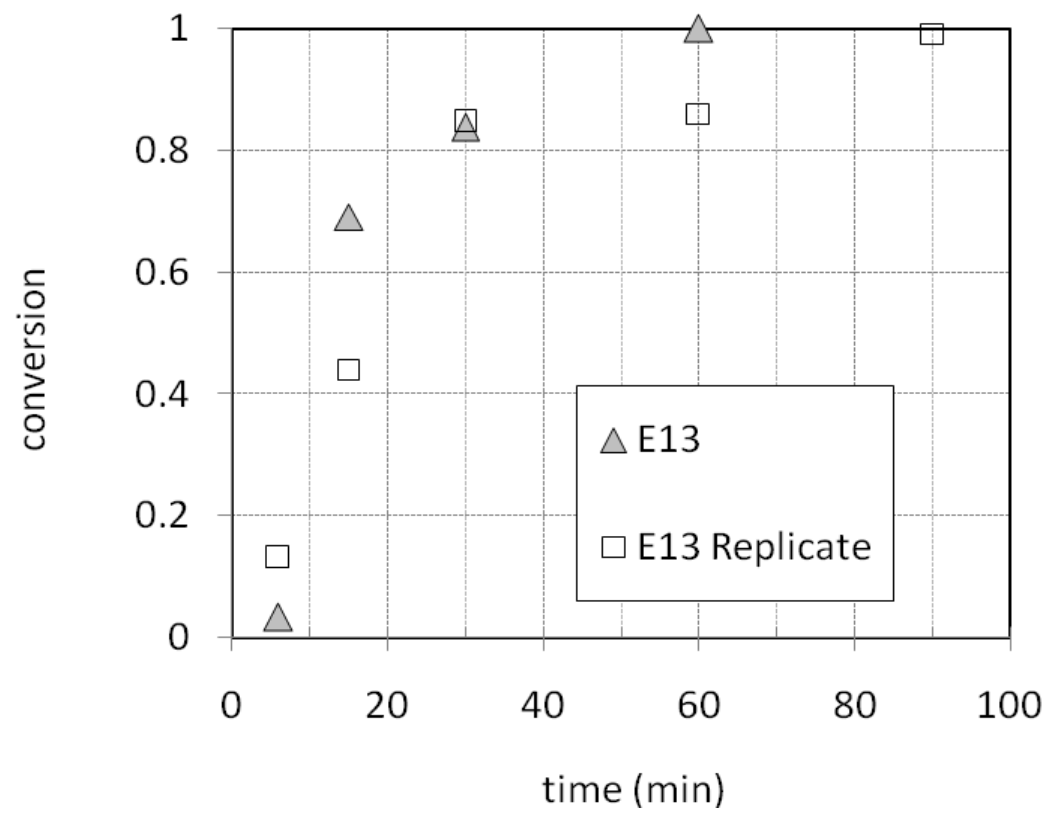

Figure 14. Conversion-time homopolymerization of acrylic acid during run E13 and run E13 replicated.

\subsubsection{Copolymerization}

In this section, experimental data of conversion for the different copolymerization runs are presented and discussed. The effects of different process variables, such as, temperature, crosslinker type, crosslinker concentration, acrylic acid concentration, initiator concentration, duration of feeding period, etc., are analyzed by comparing the results of runs performed under similar conditions, having, when possible, only significant variation of the variable under analysis (one variable at a time). 


\subsubsection{Effect of temperature}

Experiments E1, E2 and E3 were carried out at different temperatures, 70, 60 and $50^{\circ} \mathrm{C}$, respectively. The conversion-versus-time variations for these runs are presented in Figure 15.

It is evident from this figure that the duration of the induction period changes with the temperature. However, the rate of polymerization (after the end of the induction period) seems to be almost the same for the three runs.

\begin{tabular}{|l|c|c|c|c|c|c|c|c|c|c|}
\hline Run & $\begin{array}{c}\mathrm{T} \\
\left({ }^{\circ} \mathrm{C}\right)\end{array}$ & $\begin{array}{c}\text { Toluene } \\
(\mathrm{g})\end{array}$ & $\begin{array}{c}\text { Span } \\
60 \\
(\mathrm{~g})\end{array}$ & $\begin{array}{c}\text { Water } \\
(\mathrm{g})\end{array}$ & $\begin{array}{c}\text { Acrylic } \\
\text { Acid } \\
(\mathrm{g})\end{array}$ & $\begin{array}{c}\text { TMPTA } \\
(\mathrm{g})\end{array}$ & $\begin{array}{c}\text { EGDMA } \\
(\mathrm{g})\end{array}$ & $\begin{array}{c}\text { TAO } \\
(\mathrm{g})\end{array}$ & $\begin{array}{c}\text { Sodium } \\
\text { Persulfate } \\
(\mathrm{g})\end{array}$ & $\begin{array}{c}\text { Feeding } \\
\text { Time } \\
(\mathrm{min})\end{array}$ \\
\hline E1 & $\mathbf{7 0}$ & 191.18 & 3.54 & 30.8870 & 7.8443 & 0.1016 & 0 & 0 & 0.1512 & 40 \\
\hline E2 & $\mathbf{6 0}$ & 191.25 & 3.88 & 35.2797 & 7.7132 & 0.1081 & 0 & 0 & 0.15 & 40 \\
\hline E3 & $\mathbf{5 0}$ & 192.8 & 3.58 & 29.7509 & 7.714 & 0.1208 & 0 & 0 & 0.1522 & 40 \\
\hline
\end{tabular}

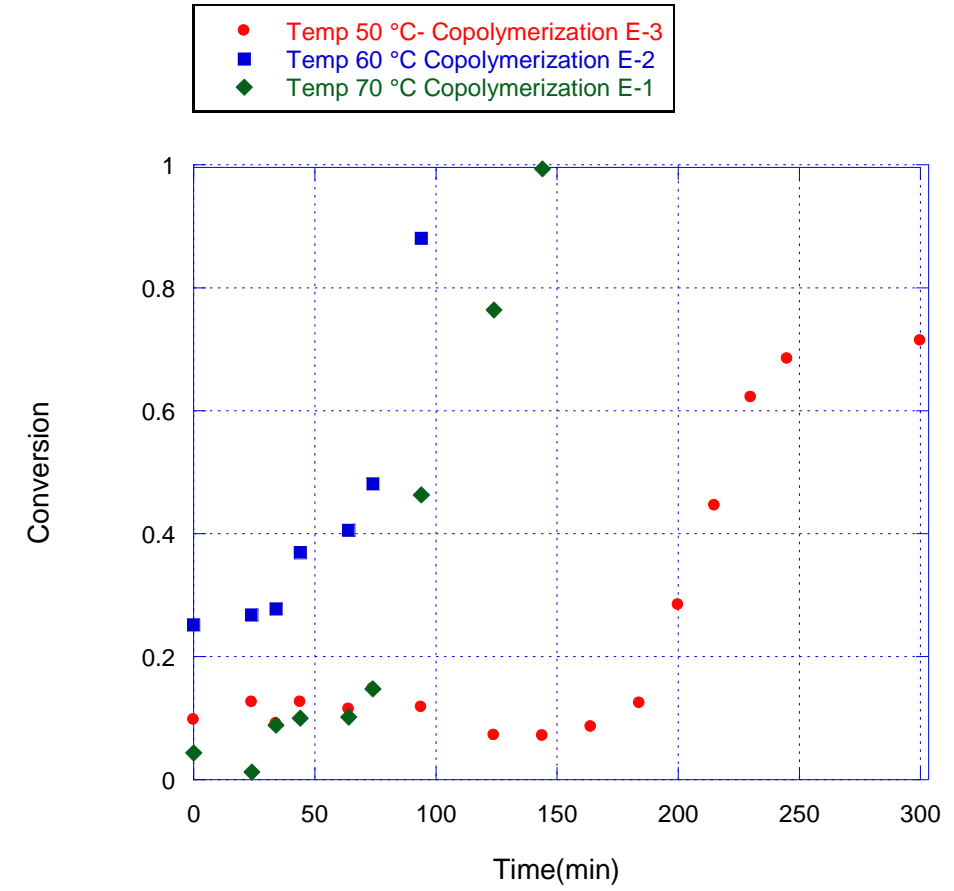

Figure 15. Conversion-time inverse suspension polymerization at different temperatures

For run E3 at $50^{\circ} \mathrm{C}$, the conversion was low ( 10-15\%) for the first $180 \mathrm{~min}$, then increased, but reached a relatively low final conversion, stopped at $\sim 72 \%$. Run $\mathrm{E} 1$ at $70^{\circ} \mathrm{C}$ presented a much shorter induction period ( 70 min) and then 
increased the polymerization rate, reaching complete conversion (100\%) at 145 minutes. These observations are in accord to the classical theory of free radical polymerization: at higher temperature, the decomposition of the initiator is faster, thus generating more radicals and consuming faster the initial amount of inhibitor, thus reducing the induction period.

Curiously, however, the complete final conversion (100\%) was obtained in even short period for run E2, which was performed at the intermediate temperature, $60^{\circ} \mathrm{C}$. Run E2 apparently had no strong induction period, as the initial conversion at time zero (i.e., at the time immediately after the feeding period) was surprisingly high ( 25\%).

\subsubsection{Effect of Crosslinker Type}

The effect of the crosslinker type was studied by performing experiments with similar conditions changing the type of crosskinking agent. The results for run E14 (with bi-functional crosslinker EGDMA), run E10 (with tri-functional crosslinker TMPTA) and run E5 (with tetra-functional crosslinker TAO) are presented in Figure 16 and 17.

In this Figure, the highest polymerization rate was observed for EGDMA and the lowest for TAO, the conversion-versus-time curve for TMPTA presenting an intermediate behavior. The direct interpretation of these results is difficult due to the differences in functionality and molar mass, as these runs were performed with about the same weight concentration of the crosslinkers. Moreover, the reactivity of the different crosslinkers with acrylic acid can be different, as well as the reactivity of the different pendant double bonds left by each of the crosslinkers.

\begin{tabular}{|l|c|c|c|c|c|c|c|c|c|c|}
\hline Run & $\begin{array}{c}\mathrm{T} \\
\left({ }^{\circ} \mathrm{C}\right)\end{array}$ & $\begin{array}{c}\text { Toluene } \\
(\mathrm{g})\end{array}$ & $\begin{array}{c}\text { Span 60 } \\
(\mathrm{g})\end{array}$ & $\begin{array}{c}\text { Water } \\
(\mathrm{g})\end{array}$ & $\begin{array}{c}\text { Acrylic } \\
\text { Acid } \\
(\mathrm{g})\end{array}$ & $\begin{array}{c}\text { TMPTA } \\
(\mathrm{g})\end{array}$ & $\begin{array}{c}\text { EGDMA } \\
(\mathrm{g})\end{array}$ & $\begin{array}{c}\text { TAO } \\
(\mathrm{g})\end{array}$ & $\begin{array}{c}\text { Sodium } \\
\text { Persulfate } \\
(\mathrm{g})\end{array}$ & $\begin{array}{c}\text { Feeding } \\
\text { Time } \\
(\mathrm{min})\end{array}$ \\
\hline E5 & 60 & 196.12 & 3.56 & 34.5494 & 7.717 & 0 & 0 & 0.057 & 0.1569 & 40 \\
\hline E10 & 60 & 191.75 & 3.6 & 30.2885 & 7.7702 & 0.05 & 0 & 0 & 0.1199 & 40 \\
\hline E14 & 60 & 193.78 & 3.72 & 30.4835 & 7.7866 & 0 & 0.0633 & 0 & 0.1976 & 40 \\
\hline
\end{tabular}




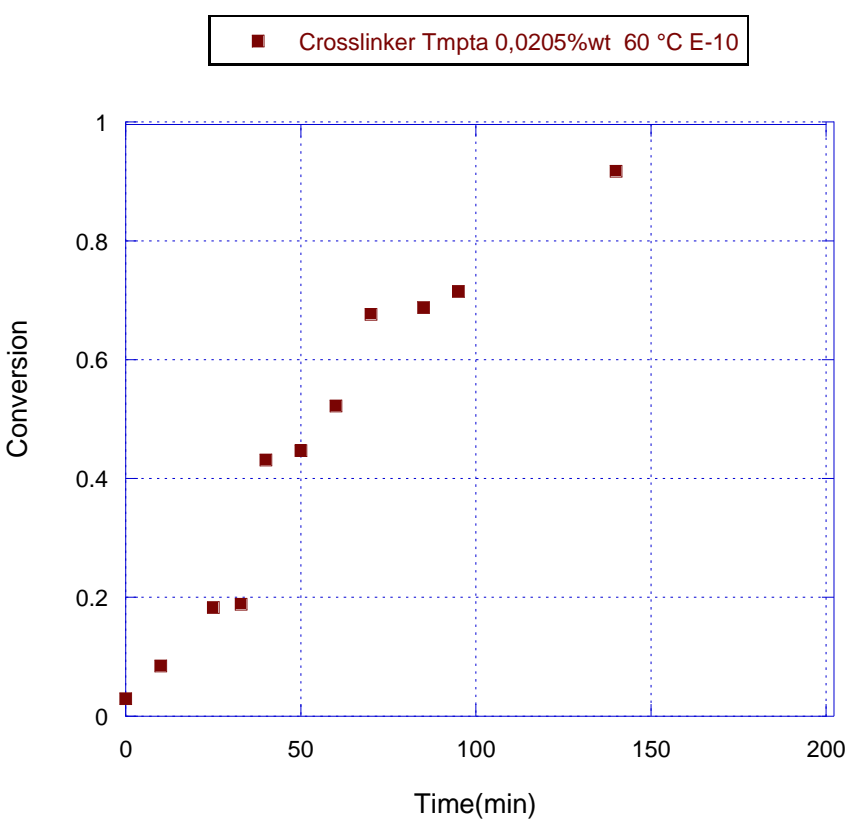

Figure 16. Conversion-time inverse suspension polymerization with TMPTA concentration Run E 10

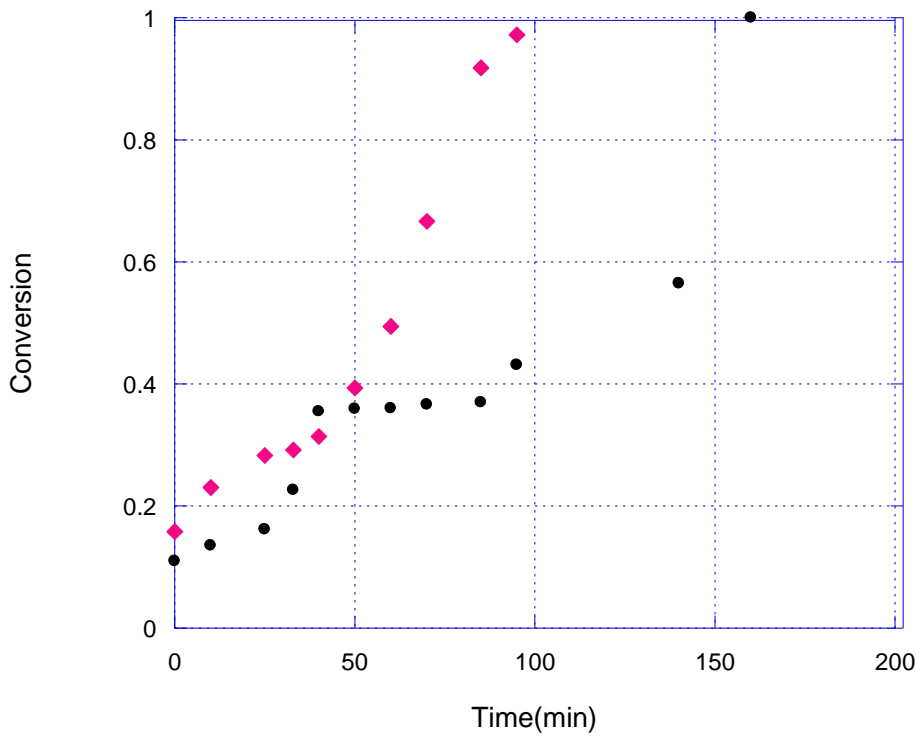

Figure 17. Conversion-time inverse suspension polymerization with different crosslinkers TAO and EGDMA Run E 15 and E 14 


\subsubsection{Effect of initiator concentration}

Figure 18 shows the effect of the initiator (sodium persulfate) concentration on the polymerization rate. The overall rate of polymerization increased with initiator concentration, as expected from the classical theory of free-radical polymerization, which predicts the rate of polymerization to be proportional to the square-root of the initiator concentration. The average polymerization rate for run E6 was $\sim 0.60 \% / \mathrm{min}$, while was $\sim 0.85 \% / \mathrm{min}$ for run E2.

Also, the delay (induction period), caused by the presence of commercial inhibitor MEHQ in the monomer, was a little longer for the run with lower initiator concentration, which is also in accord with the classical theory of free-radical polymerization.

\begin{tabular}{|l|c|c|c|c|c|c|c|c|c|c|}
\hline Run & $\begin{array}{c}\mathrm{T} \\
\left({ }^{\circ} \mathrm{C}\right)\end{array}$ & $\begin{array}{c}\text { Toluene } \\
(\mathrm{g})\end{array}$ & $\begin{array}{c}\text { Span } \\
60 \\
(\mathrm{~g})\end{array}$ & $\begin{array}{c}\text { Water } \\
(\mathrm{g})\end{array}$ & $\begin{array}{c}\text { Acrylic } \\
\text { Acid } \\
(\mathrm{g})\end{array}$ & $\begin{array}{c}\text { TMPTA } \\
(\mathrm{g})\end{array}$ & $\begin{array}{c}\text { EGDMA } \\
(\mathrm{g})\end{array}$ & $\begin{array}{c}\text { TAO } \\
(\mathrm{g})\end{array}$ & $\begin{array}{c}\text { Sodium } \\
\text { Persulfate } \\
(\mathrm{g})\end{array}$ & $\begin{array}{c}\text { Feeding } \\
\text { Time } \\
(\mathrm{min})\end{array}$ \\
\hline E2 & 60 & 191.25 & 3.88 & 35.2797 & 7.7132 & 0.1081 & 0 & 0 & 0.1500 & 40 \\
\hline E6 & 60 & 191.80 & 3.68 & 29.9852 & 7.6800 & 0.1015 & 0 & 0 & 0.0689 & 40 \\
\hline
\end{tabular}

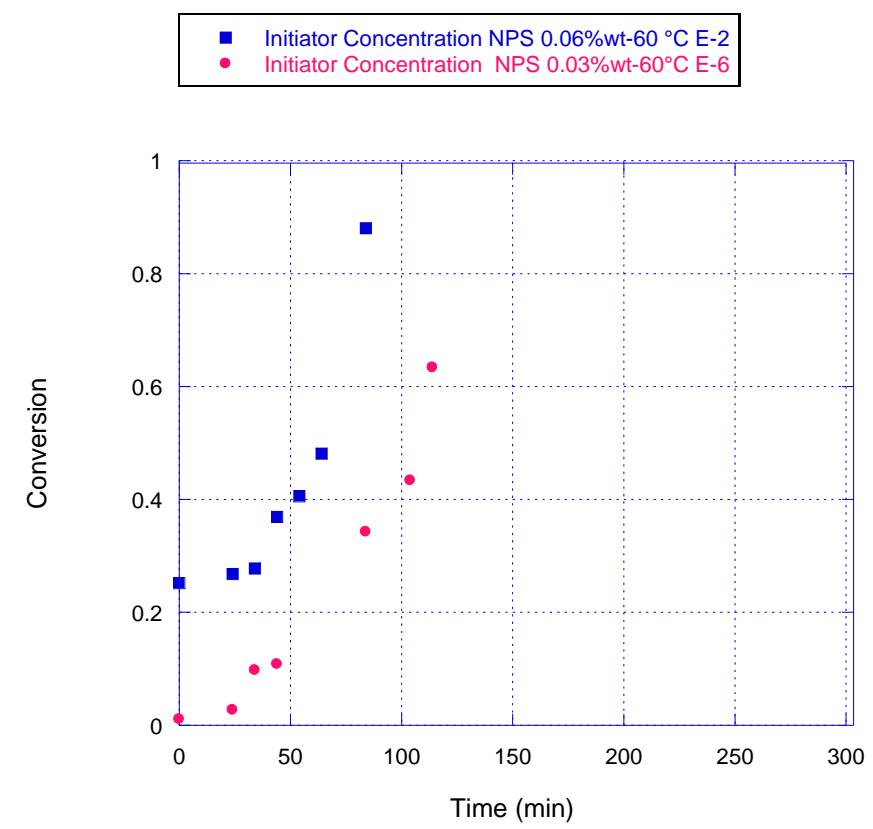

Figure 18. Conversion-time inverse suspension polymerization at different Sodium Persulfate concentration Run E 2 and E 6 


\subsubsection{Effect of the Feeding Time}

The effect of the duration of the feeding of aqueous solution (containing acrylic acid, TMPTA, sodium persulfate and water) to the reactor, before the zerotime of the batch process, was analyzed in Figure 20, in which the conversionversus-time curves for runs E2 (feeding time $40 \mathrm{~min}$ ) and E8 (feeding time $60 \mathrm{~min}$ ) are compared.

\begin{tabular}{|l|c|c|c|c|c|c|c|c|c|c|}
\hline Run & $\begin{array}{c}\mathrm{T} \\
\left({ }^{\circ} \mathrm{C}\right)\end{array}$ & $\begin{array}{c}\text { Toluene } \\
(\mathrm{g})\end{array}$ & $\begin{array}{c}\text { Span } \\
60 \\
(\mathrm{~g})\end{array}$ & $\begin{array}{c}\text { Water } \\
(\mathrm{g})\end{array}$ & $\begin{array}{c}\text { Acrylic } \\
\text { Acid } \\
(\mathrm{g})\end{array}$ & $\begin{array}{c}\text { TMPTA } \\
(\mathrm{g})\end{array}$ & $\begin{array}{c}\text { EGDMA } \\
(\mathrm{g})\end{array}$ & $\begin{array}{c}\text { TAO } \\
(\mathrm{g})\end{array}$ & $\begin{array}{c}\text { Sodium } \\
\text { Persulfate } \\
(\mathrm{g})\end{array}$ & $\begin{array}{c}\text { Feeding } \\
\text { Time } \\
(\mathrm{min})\end{array}$ \\
\hline E2 & 60 & 191.25 & 3.88 & 35.2797 & 7.7132 & 0.1081 & 0 & 0 & 0.1500 & 40 \\
\hline E8 & 60 & 190.72 & 3.80 & 31.1329 & 7.8759 & 0.1175 & 0 & 0 & 0.1546 & 60 \\
\hline
\end{tabular}

Copolymerization Feed Flow Rate $40 \mathrm{~min} 60^{\circ} \mathrm{C} \mathrm{E}-2$
Copolymerization Feed Flow Rate $60 \mathrm{~min} 60^{\circ} \mathrm{C} \mathrm{E}-8$

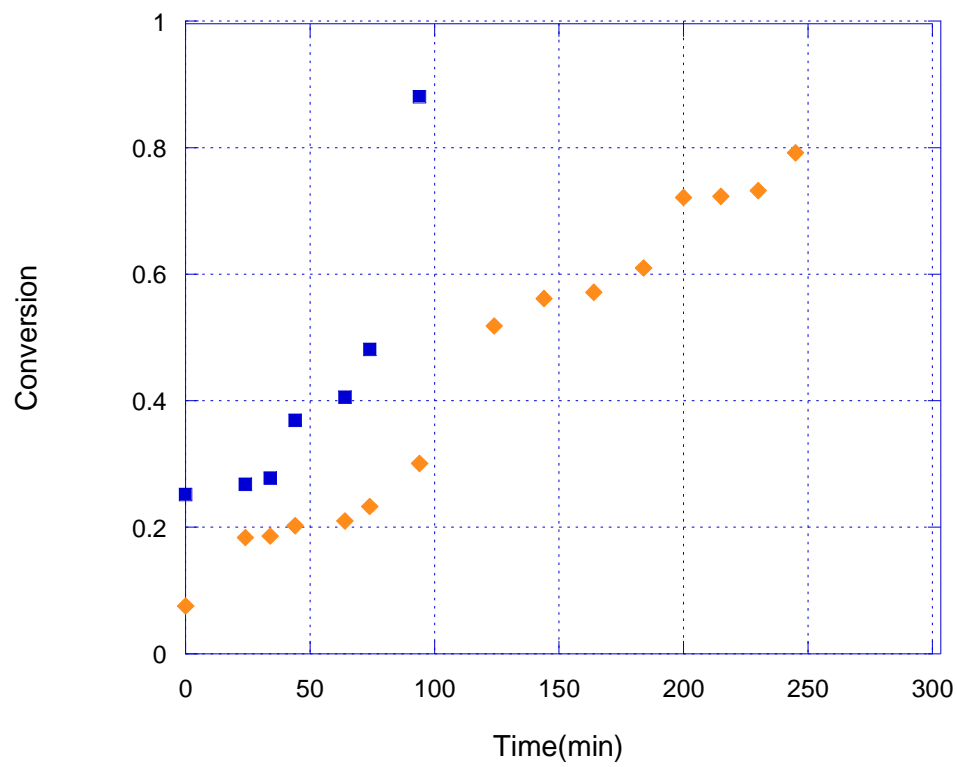

Figure 19. Conversion-time inverse suspension polymerization at different feed flow rate time Run E 2 and E 8

For run E8 (longer feeding time, $60 \mathrm{~min}$ ), no induction time is observed, but the polymerization during the batch period was slower $(0.3 \% / \mathrm{min})$, see Figure 20 . For run E2 (shorter feeding time, 40 min) Figure 21, an induction time of about 50$60 \mathrm{~min}$ was observed, followed by a period of faster polymerization $(1.0 \% / \mathrm{min})$. 
- Polymerization rate $\% \min$

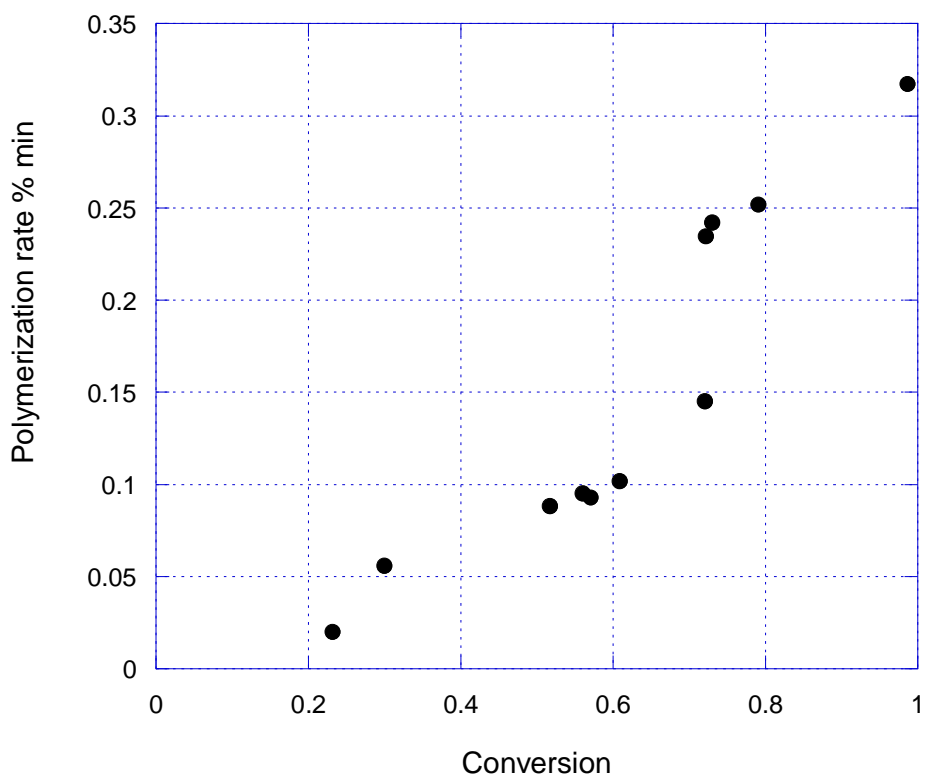

Figure 20. Conversion-Polymerization rate of inverse suspension polymerization Run E8

- Polymerization Rate \% min E2

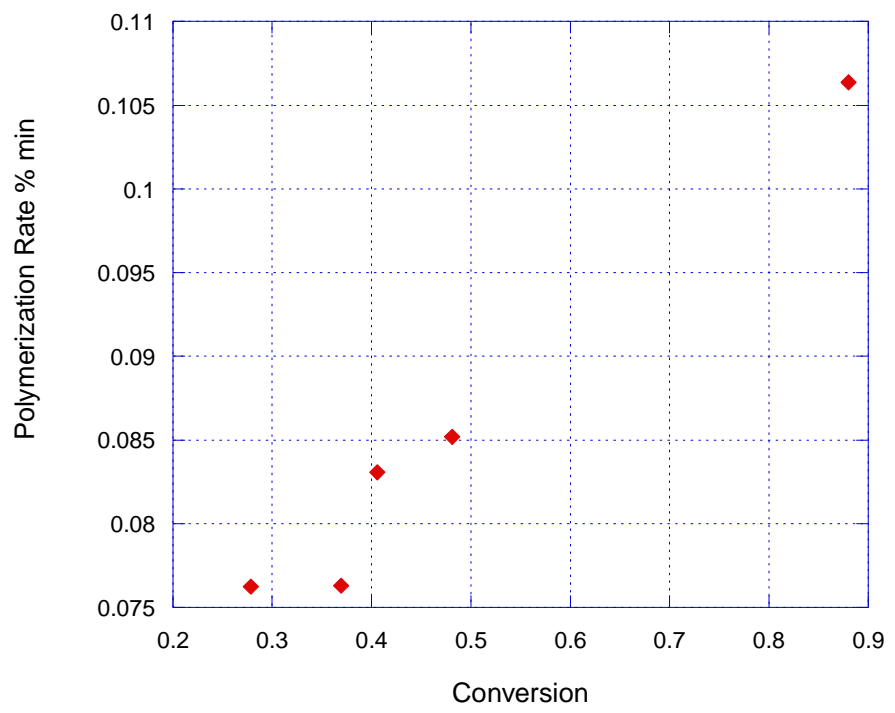

Figure 21. Conversion-Polymerization rate of inverse suspension polymerization Run E2 


\subsubsection{Effect of acrylic acid concentration}

Experiments E2 and E9 were carried out under similar conditions except the concentration of acrylic acid, which was $\sim 3.20 \mathrm{wt} \%$ in E2 and $\sim 1.20 \mathrm{wt} \%$ in E9. The results of these two runs are compared in Figure 22.

\begin{tabular}{|l|c|c|c|c|c|c|c|c|c|c|}
\hline Run & $\begin{array}{c}\mathrm{T} \\
\left({ }^{\circ} \mathrm{C}\right)\end{array}$ & $\begin{array}{c}\text { Toluene } \\
(\mathrm{g})\end{array}$ & $\begin{array}{c}\text { Span } \\
60 \\
(\mathrm{~g})\end{array}$ & $\begin{array}{c}\text { Water } \\
(\mathrm{g})\end{array}$ & $\begin{array}{c}\text { Acrylic } \\
\text { Acid } \\
(\mathrm{g})\end{array}$ & $\begin{array}{c}\text { TMPTA } \\
(\mathrm{g})\end{array}$ & $\begin{array}{c}\text { EGDMA } \\
(\mathrm{g})\end{array}$ & $\begin{array}{c}\text { TAO } \\
(\mathrm{g})\end{array}$ & $\begin{array}{c}\text { Sodium } \\
\text { Persulfate } \\
(\mathrm{g})\end{array}$ & $\begin{array}{c}\text { Feeding } \\
\text { Time } \\
(\mathrm{min})\end{array}$ \\
\hline E2 & 60 & 191.25 & 3.88 & 35.2797 & 7.7132 & 0.1081 & 0 & 0 & 0.1500 & 40 \\
\hline E9 & 60 & 190.99 & 3.61 & 26.3136 & 3.5363 & 0.1000 & 0 & 0 & 0.1546 & 40 \\
\hline
\end{tabular}

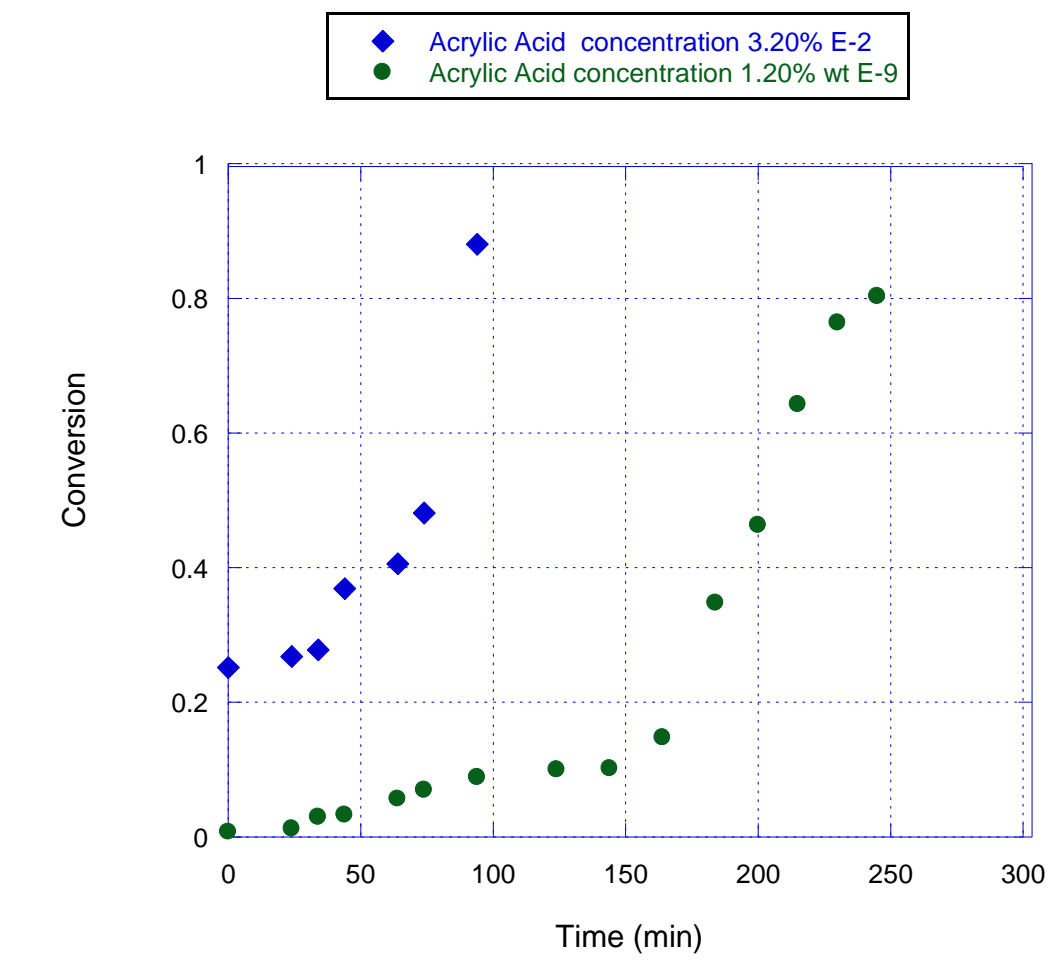

Figure 22. Conversion-time inverse suspension different acrylic acid concentration Run E2 and E9

The induction time was about 140 min for run E9 and about 30 min for run

E2. This result indicates that the induction time is caused not only by the inhibitor 
MEHQ that comes with the commercial monomers. If $M E H Q$ was the only inhibitor responsible for the induction time, the expected effect is directly proportional to the presence of oxygen and impurities. However, the opposite behavior is observed in Figure 22. Therefore, this effect is probably ascribed to the presence of oxygen as an additional inhibitor. It should be mentioned that the control of the nitrogen feeding was not very precise, so variations of the nitrogen flow between the different runs may be the cause for the differences in induction time observed.

After the end of the induction period, the polymerization occurred fast in both runs E9 and E2. The polymerization rate was $1.53 \% / \mathrm{min}$ for run E9 and $2.5 \% / \mathrm{min}$ for run E2. Comparing these values with the monomer concentration in the two runs, the apparent order for monomer was 1.6, which is in accordance to the value 1.5 reported in the literature for solution polymerization of acrylic acid (Cutié et al., 1997b; Qui et al., 2016; Lorber et al., 2010) and 1.7 for precipitation polymerization of acrylic acid (Bunyakan et al., 1999).

\subsubsection{Effect of Deoxygenation}

Experiments E15, E16, E17 and E18 were carried out to evaluate the effect of the deoxygenation period (after saturation of the initial solution with air). These experiments are similar to those performed by Cutié et al. (1997a). The aqueous solution, prepared with purified, distilled monomers (without MEHQ), was aerated by 60 minutes, then the solution was bubbled with nitrogen during different periods of time to partially deoxygenate the solution and then used in the polymerization experiments. The objective was to analyze the inhibition effect of dissolved oxygen.

The results are presented in Figures 23-26, and compared with experiment E6, performed under similar conditions but with commercial monomer (containing inhibitor MEHQ).

Figure 23 compares run E6 and run E15 (for which no deoxygenation was done). For the remaining experiments, the duration of deoxygenation time was increased progressively (15 min for E-16, 30 min for E17, 60 min for E18) and the 
results are presented in Figures 23-26. The ultimate rates of polymerization show little dependence on the deoxygenation conditions, but the magnitude of the induction time depends strongly on the deoxygenation conditions.

The presence of oxygen did not diminish (significantly) the rate of reaction, but rather lengthened the induction period, indicated that oxygen was an inhibitor rather than a retarder for this polymerization. However, slow inlet of air into the reactor (due to incomplete sealing and insufficient bubbling of inert gas) would result in a polymerization that appears retarded.

\begin{tabular}{|l|c|c|c|c|c|c|c|c|c|}
\hline Run & $\begin{array}{c}\mathrm{T} \\
{ }^{\circ} \mathrm{C}\end{array}$ & $\begin{array}{c}\text { Toluene } \\
(\mathrm{g})\end{array}$ & $\begin{array}{c}\text { Span } \\
60 \\
(\mathrm{~g})\end{array}$ & $\begin{array}{c}\text { Water } \\
(\mathrm{g})\end{array}$ & $\begin{array}{c}\text { Acrylic } \\
\text { Acid } \\
(\mathrm{g})\end{array}$ & $\begin{array}{c}\text { TMPTA } \\
(\mathrm{g})\end{array}$ & $\begin{array}{c}\text { Deoxygenation } \\
\text { Period } \\
(\mathrm{min})\end{array}$ & $\begin{array}{c}\text { Sodium } \\
\text { Persulfate } \\
(\mathrm{g})\end{array}$ & $\begin{array}{c}\text { Feeding } \\
\text { Time } \\
(\mathrm{min})\end{array}$ \\
\hline E6 & 60 & 191.8 & 3.68 & 29.9852 & 7.68 & 0.1015 & --- & 0.0689 & 40 \\
\hline E15 & 60 & 191.8 & 3.68 & 29.9852 & 7.68 & 0.1015 & 0 & 0.0689 & 40 \\
\hline E16 & 60 & 191.8 & 3.68 & 29.9852 & 7.68 & 0.1015 & 15 & 0.0689 & 40 \\
\hline E17 & 60 & 191.8 & 3.68 & 29.9852 & 7.68 & 0.1015 & 30 & 0.0689 & 40 \\
\hline E18 & 60 & 191.8 & 3.68 & 29.9852 & 7.68 & 0.1015 & 60 & 0.0689 & 40 \\
\hline
\end{tabular}

E-6
$\square \quad$ No Deox- E 15

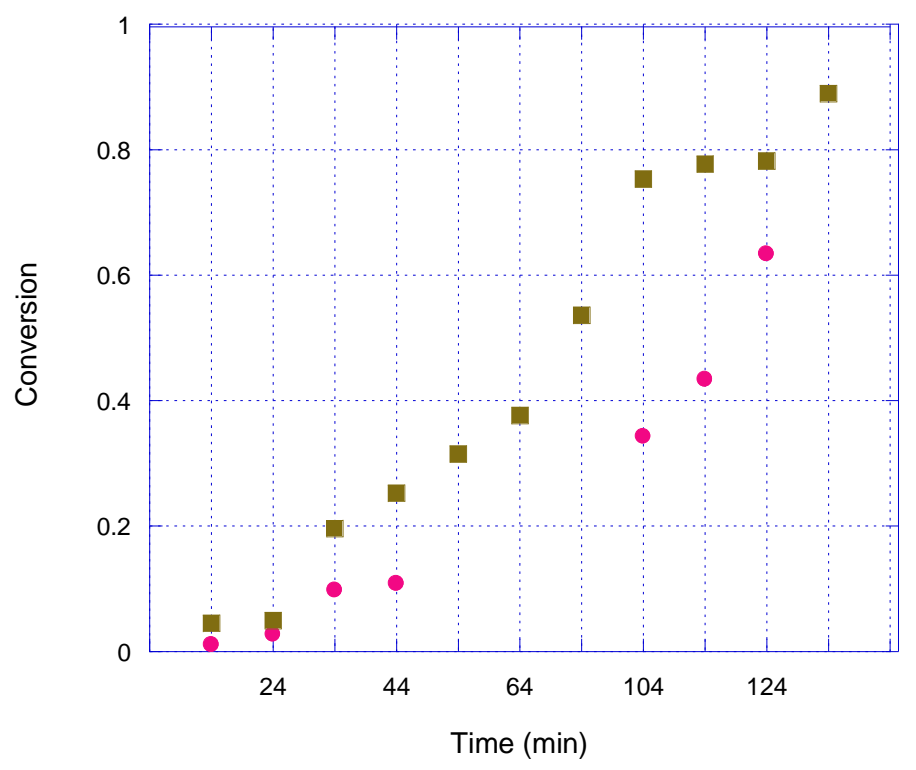

Figure 23. Polymerization rate and inhibition time as a function of deoxygenation conditions. No deoxygenation 


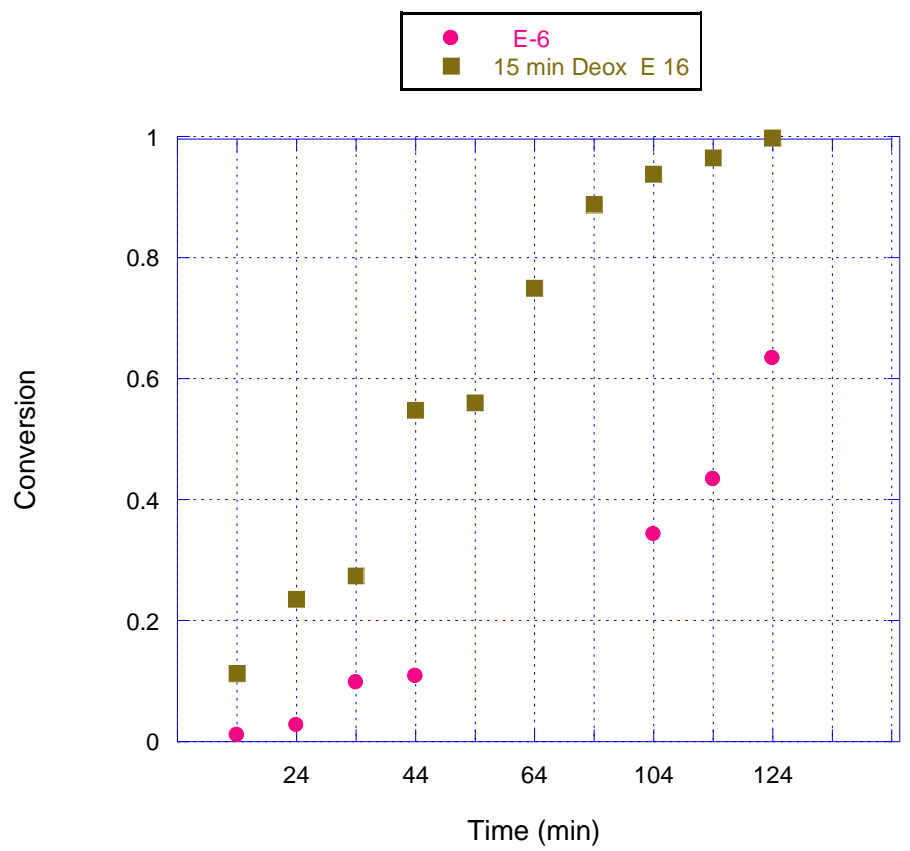

Figure 24. Polymerization rate and inhibition time as a function of deoxygenation conditions. 15 min deoxygenation

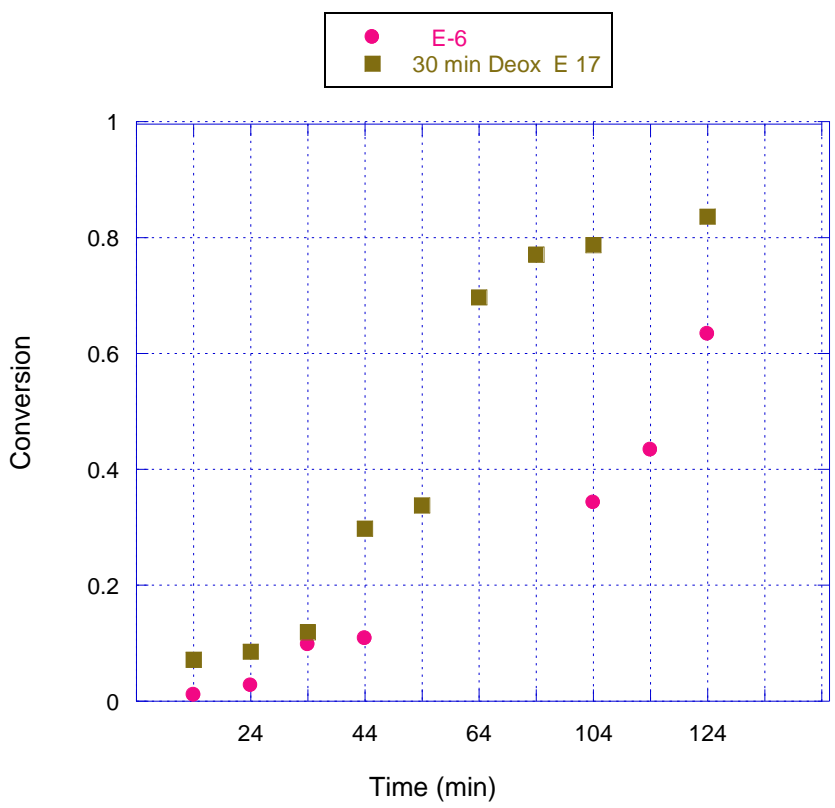

Figure 25. Polymerization rate and inhibition time as a function of deoxygenation conditions. 30 min deoxygenation 


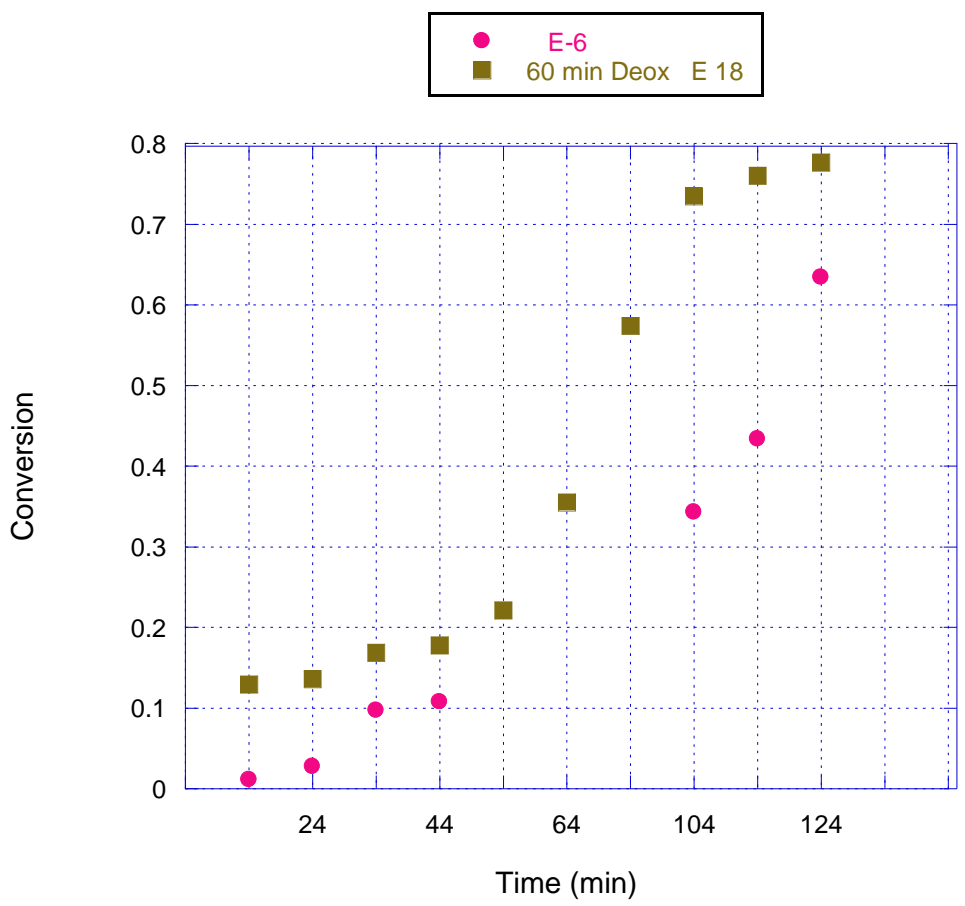

Figure 26. Polymerization rate and inhibition time as a function of deoxygenation conditions. 60 min deoxygenation

\subsection{Gel Fraction}

The gel fraction was measured in the dried polymer samples obtained by the measurement of conversion by gravimetry. Gel fraction was measured by a 3-hour extraction with solvent (water) in a Soxtherm equipment. This measurement was performed in runs E5, E10 and E14 in which different crosslinker types were tested.

\subsubsection{Crosslinker agents}

Figures 27-29 presents the results of the gel fraction in runs with different multifunctional crosslinker types, EGDMA (bi-function, run E14), TMPTA (trifunctional, run E5) and TAO (tetarfunctional, run E10). 
A growing trend of the gel fraction with time is noted in Figure 27, which is consistent with the experimental conditions with the increase in the initial concentration of monomers. The analysis of the gel fraction becomes less accurate when the amount of polymer analyzed decreases (i.e., for low conversions of monomer to polymer). The samples collected early in the reactions still possess little polymer, which reduces the precision of the analysis.

Figure 28 shows that the weight fraction of gel with TAO was higher than with EGDMA, demonstrating the effect of the increase of the crosslinker functionality in the copolymerization system.

Figure 29 shows the gel fraction as a function of the monomer conversion for runs E5 (TAO) and E14 (EGDMA). As expected, the gel point (i.e., the point at which the gel starts to be formed) occurs for lower conversion for the crosslinker with higher functionality.

\begin{tabular}{|l|c|c|c|c|c|c|c|c|c|c|}
\hline Run & $\begin{array}{c}\mathrm{T} \\
\left({ }^{\circ} \mathrm{C}\right)\end{array}$ & $\begin{array}{c}\text { Toluene } \\
(\mathrm{g})\end{array}$ & $\begin{array}{c}\text { Span 60 } \\
(\mathrm{g})\end{array}$ & $\begin{array}{c}\text { Water } \\
(\mathrm{g})\end{array}$ & $\begin{array}{c}\text { Acrylic } \\
\text { Acid } \\
(\mathrm{g})\end{array}$ & $\begin{array}{c}\text { TMPTA } \\
(\mathrm{g})\end{array}$ & $\begin{array}{c}\text { EGDMA } \\
(\mathrm{g})\end{array}$ & $\begin{array}{c}\text { TAO } \\
(\mathrm{g})\end{array}$ & $\begin{array}{c}\text { Sodium } \\
\text { Persulfate } \\
(\mathrm{g})\end{array}$ & $\begin{array}{c}\text { Feeding } \\
\text { Time } \\
(\mathrm{min})\end{array}$ \\
\hline E5 & 60 & 196.12 & 3.56 & 34.5494 & 7.717 & 0 & 0 & 0.057 & 0.1569 & 40 \\
\hline E10 & 60 & 191.75 & 3.6 & 30.2885 & 7.7702 & 0.05 & 0 & 0 & 0.1199 & 40 \\
\hline E14 & 60 & 193.78 & 3.72 & 30.4835 & 7.7866 & 0 & 0.0633 & 0 & 0.1976 & 40 \\
\hline
\end{tabular}

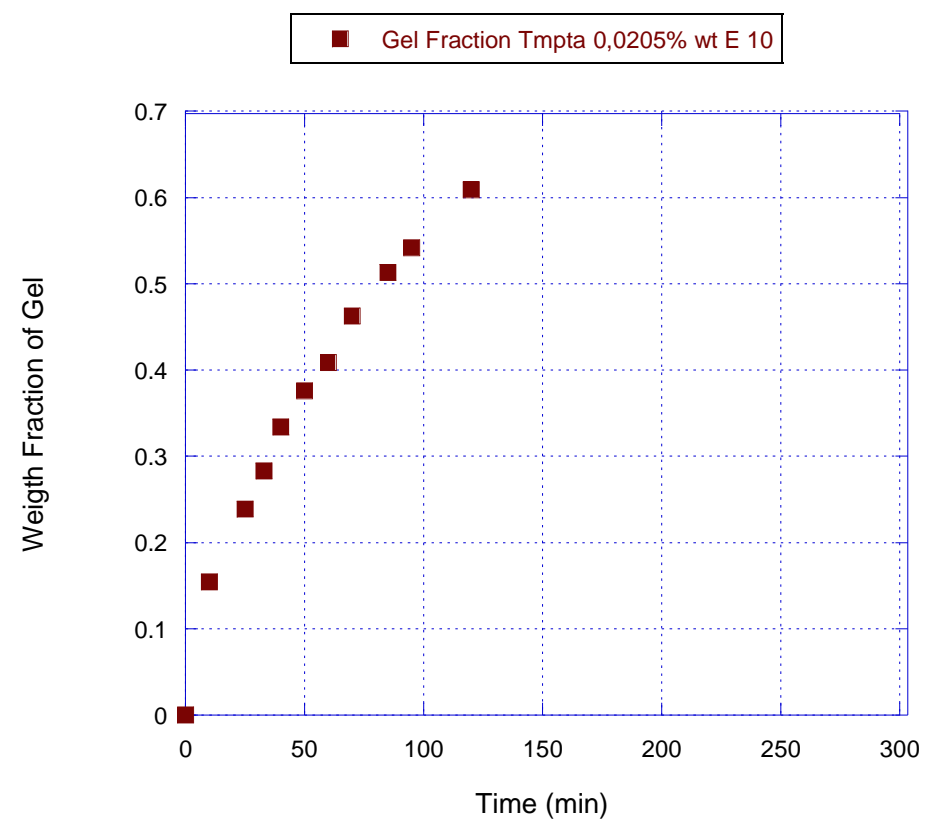

Figure 27. Weight Fraction of Gel at different Crosslinkers concentration TMPTA 0.0205 


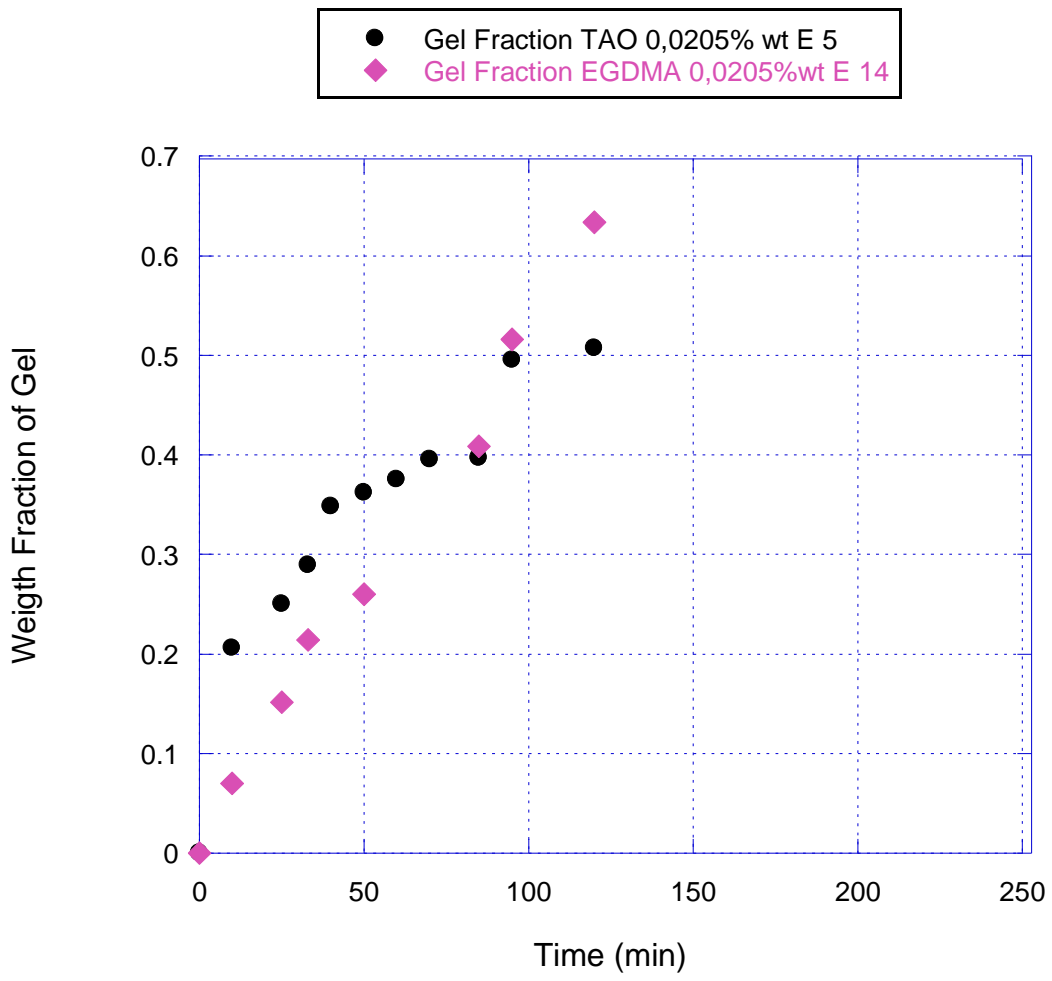

Figure 28. Weight Fraction of Gel at different Crosslinkers concentration TAO and EGDMA $0.0205 \%$

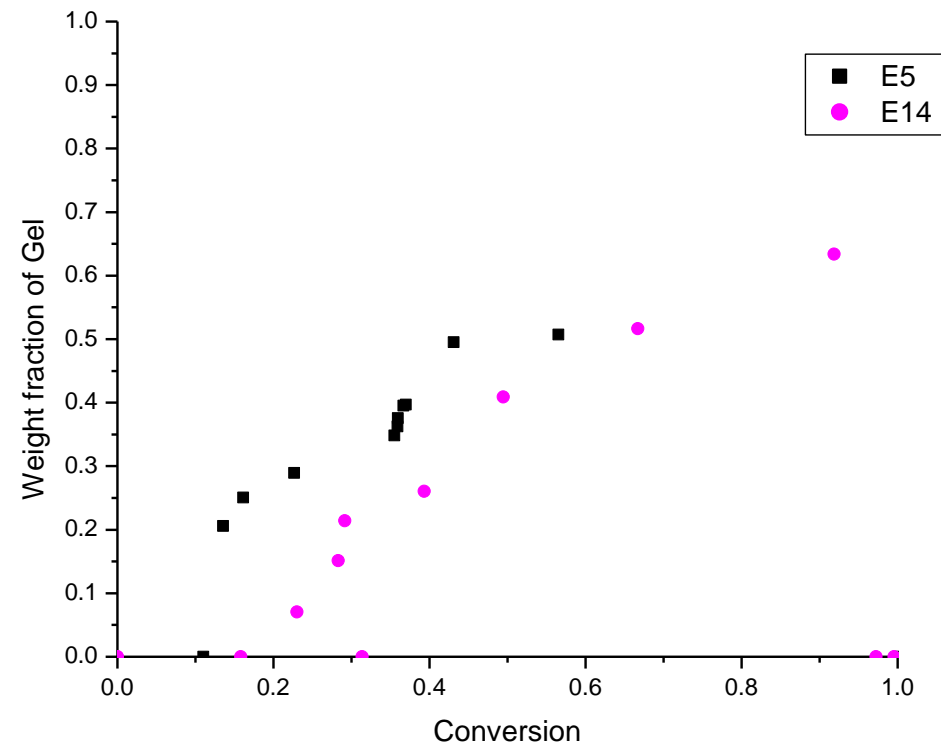

Figure 29. Comparison between experimentally observed and predicted dynamics of monomer conversion and weight fraction of gel during the copolymerization of acrylic acid and two different crosslinker with tetraallyloxyethane and Etheylene glycol dimetricrylate 


\subsection{Simulation results and comparison with experimental data}

In this section, the results of the model simulation are compared with the experimental data.

Table 5 shows the values of the kinetic parameters used in the model developed in the present work.

Table 5. Kinetic parameters used in the preliminary analysis of the mathematical model developed

\begin{tabular}{|c|c|c|}
\hline Reaction & Kinetic Parameters & Unit \\
\hline $\begin{array}{l}\text { Initiator Decomposition } \\
\text { (Sodium persulfate) }\end{array}$ & $\begin{array}{l}k_{d}=1,433 * 10^{10} \operatorname{Exp}\left(\frac{-22600}{1,987 * T}\right) \\
\boldsymbol{f}=\mathbf{0 , 7}\end{array}$ & $s^{-1}$ \\
\hline Initiation & $k_{l j}=k_{p 1 j}(1 \leq j \leq 5)$ & L. $\mathrm{mol}^{-1} \mathrm{~s}^{-1}$ \\
\hline Chain Transfer for polymer & $k_{f r}=0$ & L. $\mathrm{mol}^{-1} \mathrm{~s}^{-1}$ \\
\hline Hydrogen Abstration & $k_{h}=0$ & L. $\mathrm{mol}^{-1} \mathrm{~s}^{-1}$ \\
\hline Termination by combination & $k_{t c}=5 * 10^{7}$ & L. $\mathrm{mol}^{-1} \mathrm{~s}^{-1}$ \\
\hline Termination by disproportionation & $k_{t d}=0$ & L. $\mathrm{mol}^{-1} \mathrm{~s}^{-1}$ \\
\hline${ }^{\star}$ Propagation rate constant $K_{p 11}$ & $\begin{array}{l}k_{p 11}=\operatorname{var} \text { iavel },\left({ }^{*}\right) \\
\text { being } \\
k_{p i 1}=k_{p 11}(1 \leq i \leq 5)\end{array}$ & L. $\mathrm{mol}^{-1} \mathrm{~s}^{-1}$ \\
\hline $\begin{array}{l}{ }^{*} \text { Crosslinker Reactivity ratios } \\
r_{31}=\mathrm{kp}_{13} / \mathrm{kp}_{11} \\
\mathrm{r}_{43}=\mathrm{kp}_{43} / \mathrm{kp}_{13}\end{array}$ & $\begin{array}{l}r_{31}=0.3 \\
r_{43}=0.1 \\
r_{31}=2.31 \\
r_{43}=0.25\end{array}$ & \\
\hline
\end{tabular}

As presented in Chapter 3, the model was developed on the basis of the recent study by Gonçalves et al. (2011) to simulated the synthesis of acrylic acid hydrogels using a kinetic model based on the population balance equations and generating functions in order to predict the changes in the properties like the gel fraction and monomer conversion. 


\subsubsection{Effect of the value of the propagation rate constant $\left(\mathbf{k p}_{11}\right)$}

Gonçalves et al. (2011) reported some values of propagation kinetic constant (Tables 7 and 8 ), and its dependency of temperature and monomer concentration.

Table 6 . Reported values of the termination rate constant $\left(k_{p 11}\right)$ of acrylic acid in water

Some reported values of the termination rate constant $\left(k_{t}\right)$ of acrylic acid (AA) in water.

\begin{tabular}{|c|c|c|c|c|c|}
\hline $\mathrm{k}_{\mathrm{t}}\left(\mathrm{L} \mathrm{mol} \mathrm{m}^{-1} \mathrm{~s}^{-1}\right)$ & $\mathrm{T}\left({ }^{\circ} \mathrm{C}\right)$ & $\mathrm{pH}$ & {$[\mathrm{M}]$} & Remarks & Ref. \\
\hline $\begin{array}{l}\sim 3 \times 10^{3} \text { to } \sim 5 \times 10^{4} \\
\text { Very low values }\left(\mathrm{k}_{\mathrm{t}}=0.1 \text { to } 1\right) \\
\text { at high conversion }(\sim 0.9) \\
\text { and } \mathrm{pH}=6.5\end{array}$ & Room & $2.2-9.9$ & $30 \%$ wt AA & $\begin{array}{c}\text { Minimum at } \mathrm{pH} \sim 6.5 \\
k_{p} \downarrow \text { with conversion } \\
k_{p} \downarrow \text { with ionic strength }\end{array}$ & {$[5]$} \\
\hline$\frac{k_{p}}{\sqrt{k_{t}}}=25$ to 40 & 70 & $75 \% \mathrm{~N}$. & & Inverse Suspension & [25] \\
\hline $3 \times 10^{8}$ & 55 & $65 \% \mathrm{~N}$. & $\begin{array}{c}33 \% \text { solids }(\mathrm{AA}) \\
(\sim 25 \% \text { wt AA) }\end{array}$ & $\begin{array}{c}\text { Estimated from experimental. } \\
\text { data in Ref. [22] }\end{array}$ & [21] \\
\hline
\end{tabular}

Ref: (Gonçalves et al., 2011)

For generality, no particular chemical system has been selected for this study, but the chosen kinetic parameters should allow a simulation as much as possible close to the real behavior of the crosslinking copolymerization systems. A basic set of kinetic parameters were fixed in the calculations in order to have a realistic time evolution of the copolymerization; they are collected in the supplementary material.

The comparison of the monomer conversion profile (AA) between the model and the experimental data is presented in Figures 30 to 43, showing the effect of the variation of the propagation rate constant $\left(k_{p 11}\right)$. 
Table 7. Reported values of the propagation rate constant $\left(k_{\mathrm{p} 11}\right)$ of acrylic acid in water

Some reported values of the propagation rate constant $\left(k_{p}\right)$ of acrylic acid (AA) in water.

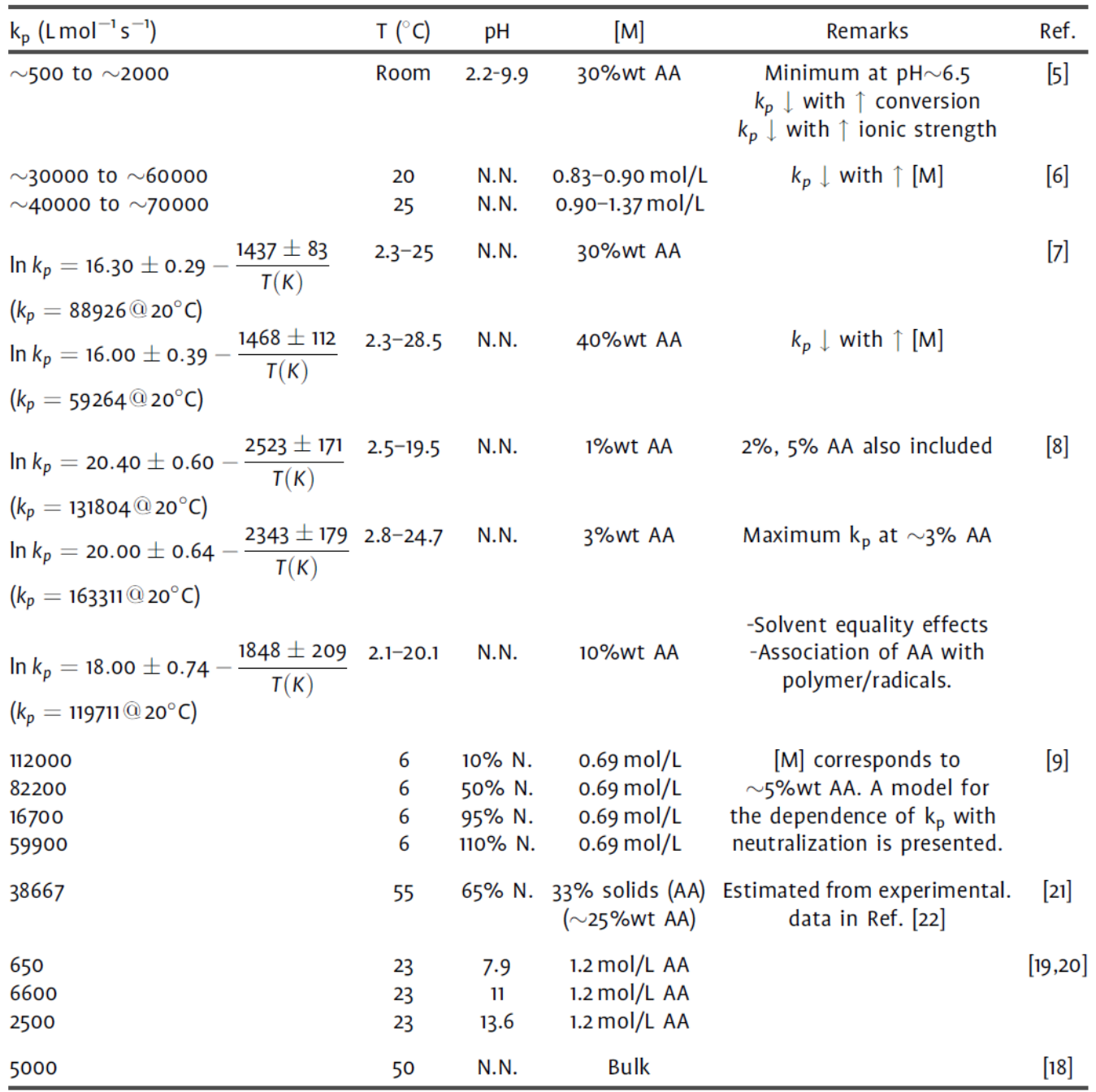

Without estimation of $k_{p}$, important kinetic data concerning the aqueous polymerization of AA or AA/TMPTA. is presented in Refs. ${ }^{4,22-24}$ The effect of neutralization of AA and solids content (dilution related) was extensively studied in these works in the temperature range 55 to $85^{\circ} \mathrm{C} . \mathrm{R}=8.314 \mathrm{Jmol}^{-1} \mathrm{~K}^{-1}$.

Ref: (Gonçalves et al., 2011) 


\subsubsection{Simulation of Homopolymerization}

Figure 30 shows the comparison of the simulation results with the experimental data of monomer conversión for the homopolymerization of acrylic acid during run E12. Because the effect of inhibitor was not explicitly accounted for in the model, in the simulation, an induction time of 30 minutes, based on the experimental data, was assumed. In order to make the simulation results closer to the experimental data, the parameter $\mathrm{K}_{\mathrm{P} 11}$, the propagation rate constant for acrylic acid, was adjusted to a value equal to $1200 \mathrm{~L} /(\mathrm{mol} . \mathrm{s})$. This value allowed fitting reasonably the trend of the experimental data, except for the last two points that indicated an autoacceleration effect ("gel effect", or diffusion-controlled termination).

The procedure for model adjusting was simply by repeated simulations and trial-and-error. The model could be further improved by refining the search of the parameter value by a formal optimization routine and by include the autoacceleration effect (diffusion-controlled termination), but this was not employed in the present work.

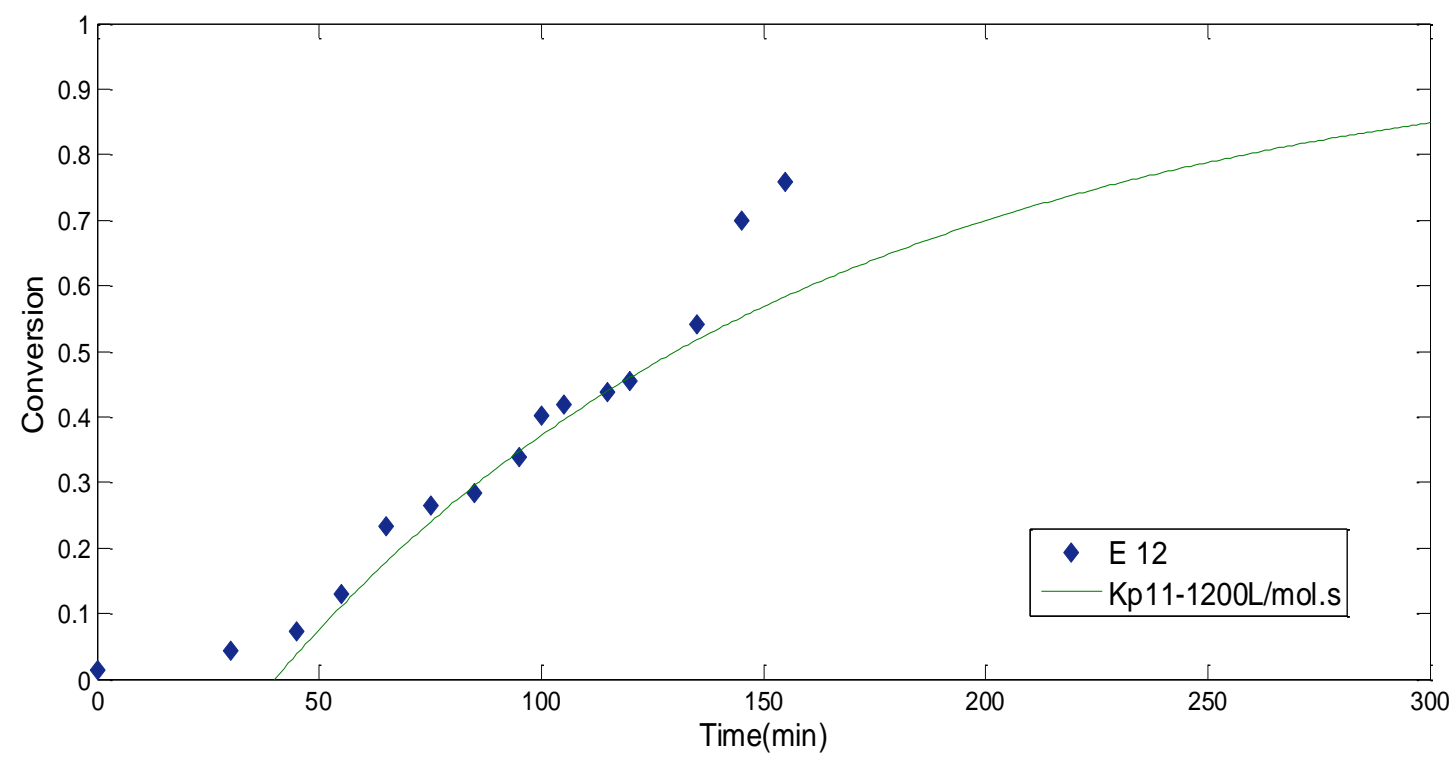

Figure 30.Conversion-time Homopolymerization process at $60^{\circ} \mathrm{C}$ 


\subsubsection{Copolymerization Simulation}

\subsubsection{Reaction Temperature}

Figure 31 to 33 shows the comparison between model results and experimental data for the runs E1, E2 and E3 carried out at different temperatures $\left(50\right.$ to $70^{\circ} \mathrm{C}$ ). The procedure included, again, the shifting of the simulated curves by an induction time obtained directly from the experimental data. In run E2 $\left(70{ }^{\circ} \mathrm{C}\right.$, Figure 31), the induction time was assumed to be 75 minutes and the fitted value of $\mathrm{K}_{\mathrm{P} 11}$ was $6000 \mathrm{~L} /(\mathrm{mol} . \mathrm{s})$. For run $\mathrm{E} 2\left(60^{\circ} \mathrm{C}\right.$, Figure 32$)$, no induction time was needed and the fitted value of $\mathrm{K}_{\mathrm{P} 11}$ was $5000 \mathrm{~L} /(\mathrm{mol} . \mathrm{s})$. For run $\mathrm{E} 3\left(50{ }^{\circ} \mathrm{C}\right.$, Figure 33) the induction time assumed was 180 minutes and the adjusted value of $K_{P 11}$ was $4500 \mathrm{~L} /(\mathrm{mol} . \mathrm{s})$.

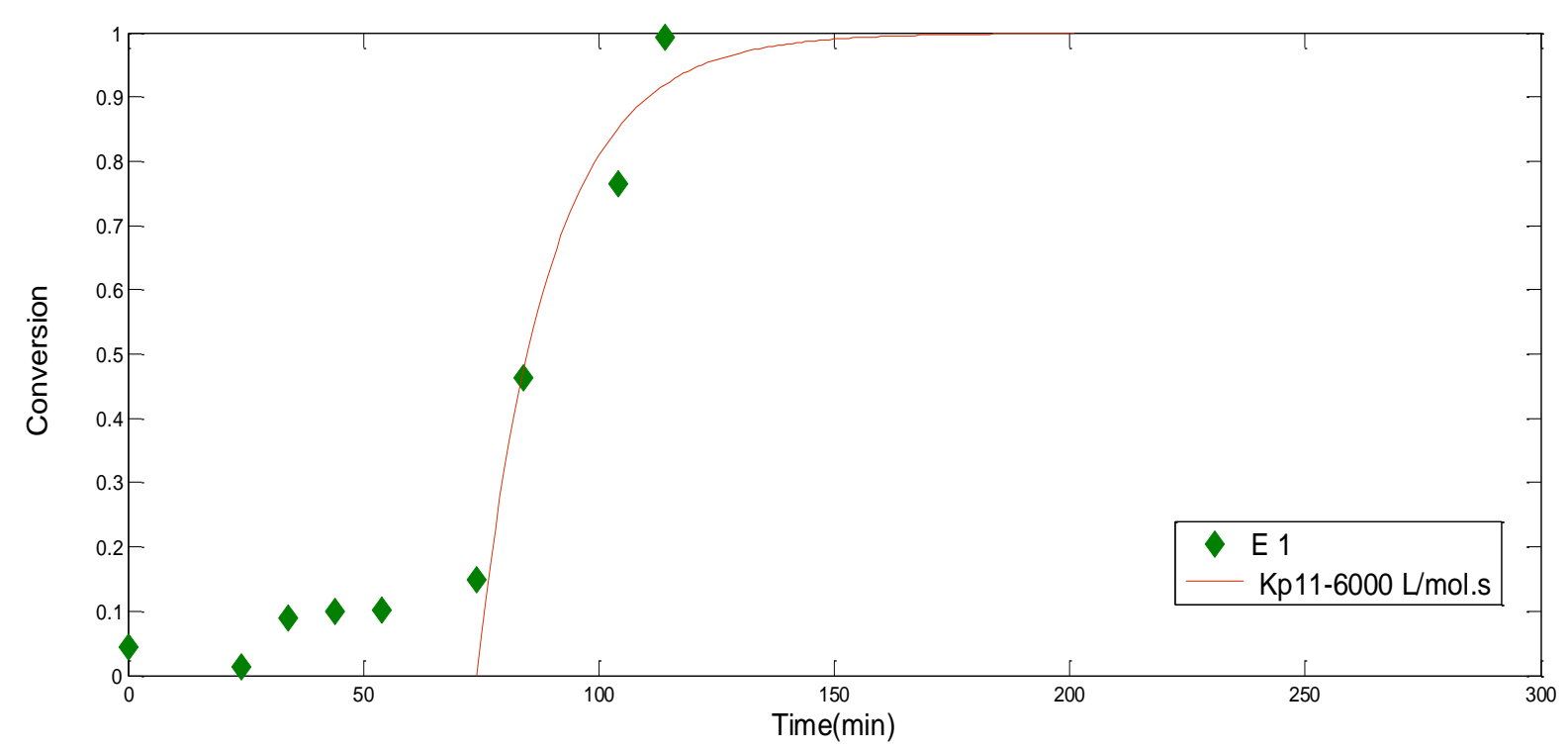

Figure 31.Conversion-time dependence of the rate coefficient for the homopropagation of acrylic acid $\mathrm{Kp}_{11}$ $70^{\circ} \mathrm{C}$ 


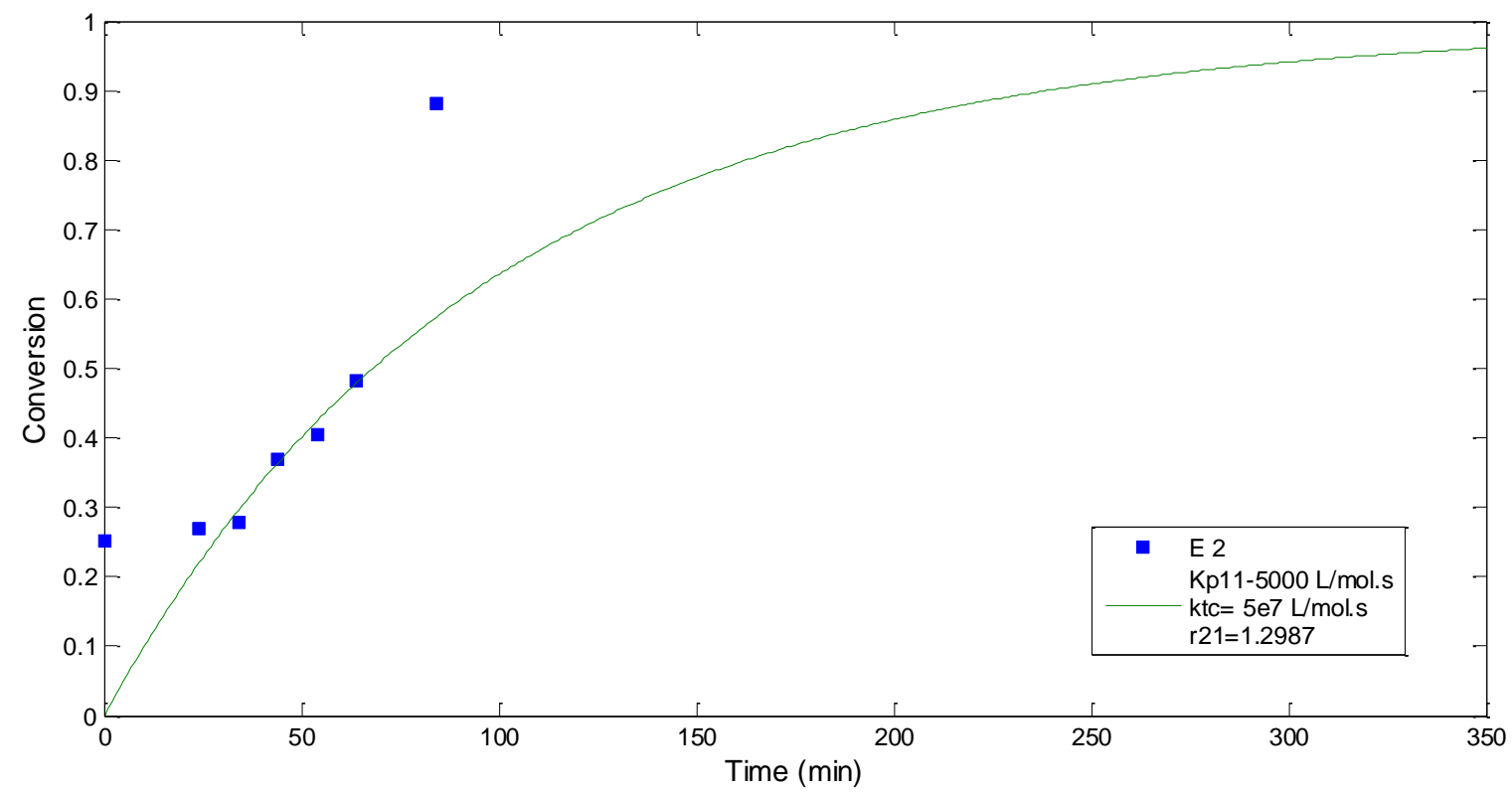

Figure 32. Conversion-time dependence rate coefficient for the homopropagation of acrylic acid $\mathrm{Kp}_{11} 60^{\circ} \mathrm{C}$

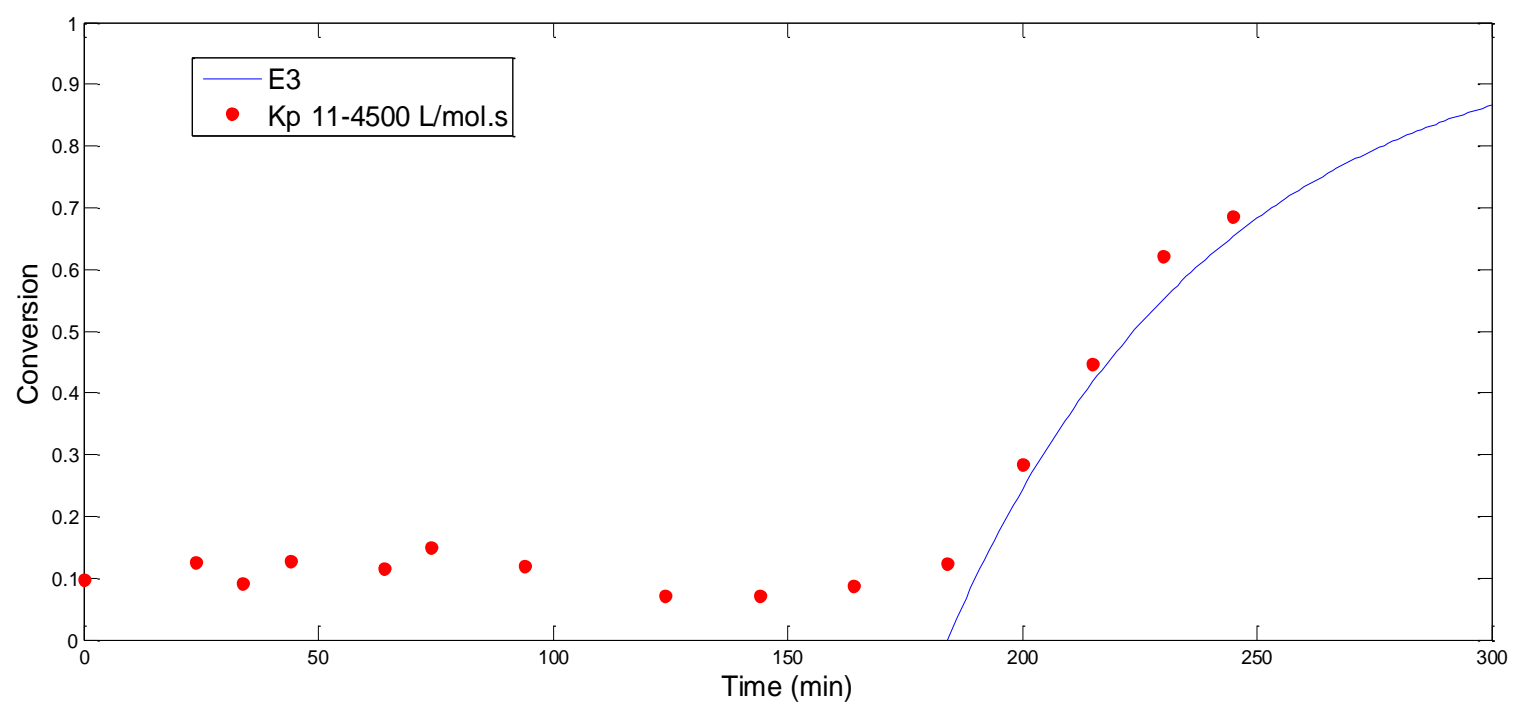

Figure 33. Conversion-Time different values of the rate coefficient for the homopropagation of acrylic acid $\mathrm{Kp}_{11}$ $50^{\circ} \mathrm{C}$

No clear explanation can be drawn from the values of the induction time, which is known to depend on the presence of inhibitors MEHQ (which comes in the commercial monomers used) and oxygen (which can be dissolved in the aqueous solution). Although nitrogen was bubbled in all experiments to deoxygenate the reaction medium, the nitrogen flow rate was not precisely controlled nor was equal 
in all experiments. This is probably the reason for the differences in induction time observed experimentally.

On the other hand, the values of $\mathrm{K}_{\mathrm{P} 11}$, manipulated to fit the experimental trend, showed the expected tendency of increase progressively with the increasing in temperature.

\subsubsection{Crosslinkers}

Figures 34 to 36 show the adjusted model curves for runs E5 (crosslinker TAO), E10 (crosslinker TMPTA) and E14 (crosslinker EGDMA). In these runs, no strong induction time was observed and then the induction time was assumed to be zero in the simulations. The values of adjusted KP11 were in the range 1500$3000 \mathrm{~L} /(\mathrm{mol} . \mathrm{s})$. Although the adjust was reasonable, no clear trend was observed between the values of $\mathrm{K}_{\mathrm{P} 11}$ and the functionality of the crosslinker $(1500 \mathrm{~L} /(\mathrm{mol} . \mathrm{s})$ for TAO, $3000 \mathrm{~L} /(\mathrm{mol} . \mathrm{s})$ for TMPTA and $2000 \mathrm{~L} /(\mathrm{mol} . \mathrm{s})$ for EGDMA), reflecting the experimental tendency of the experimental conversion-versus-time curves.

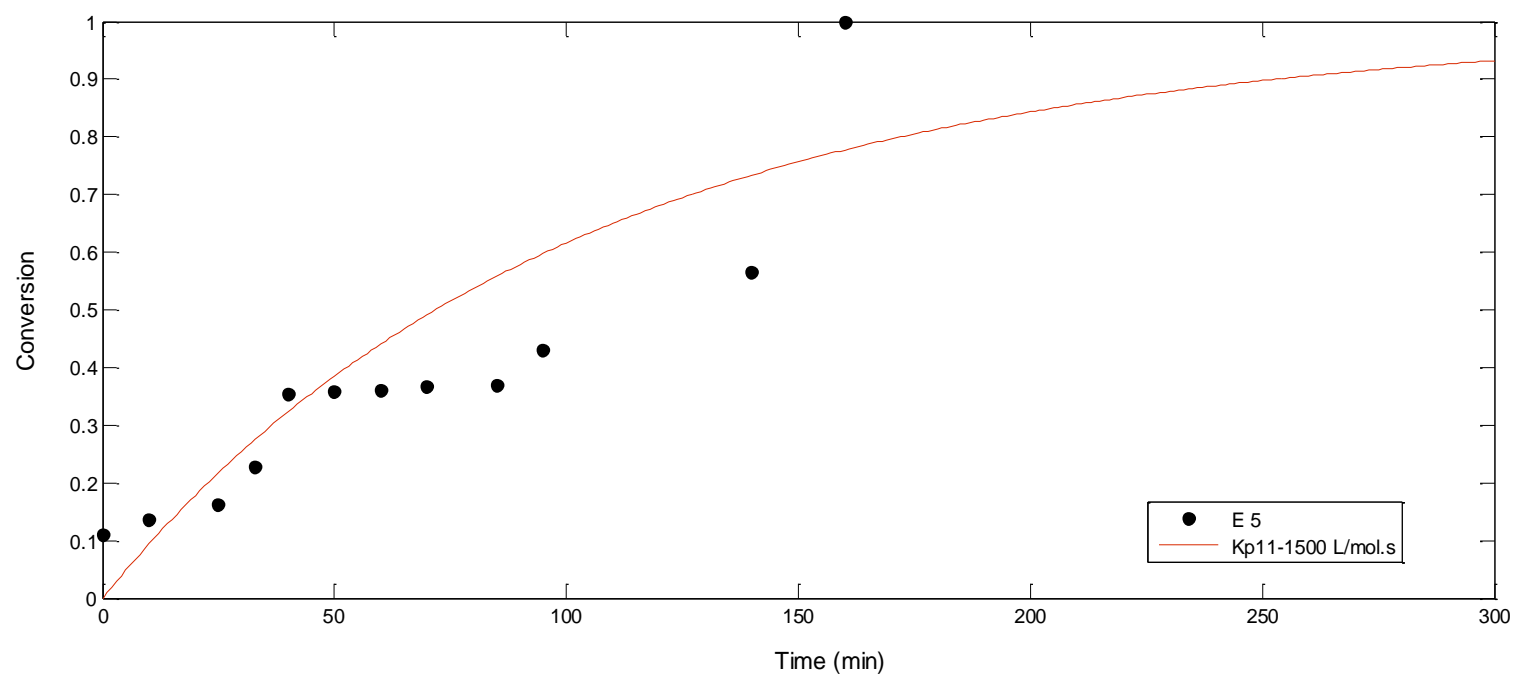

Figure 34. Conversion-time different values of rate coefficient homopropagation of acrylic acid (Kp11) influence of crosslinkers TAO 


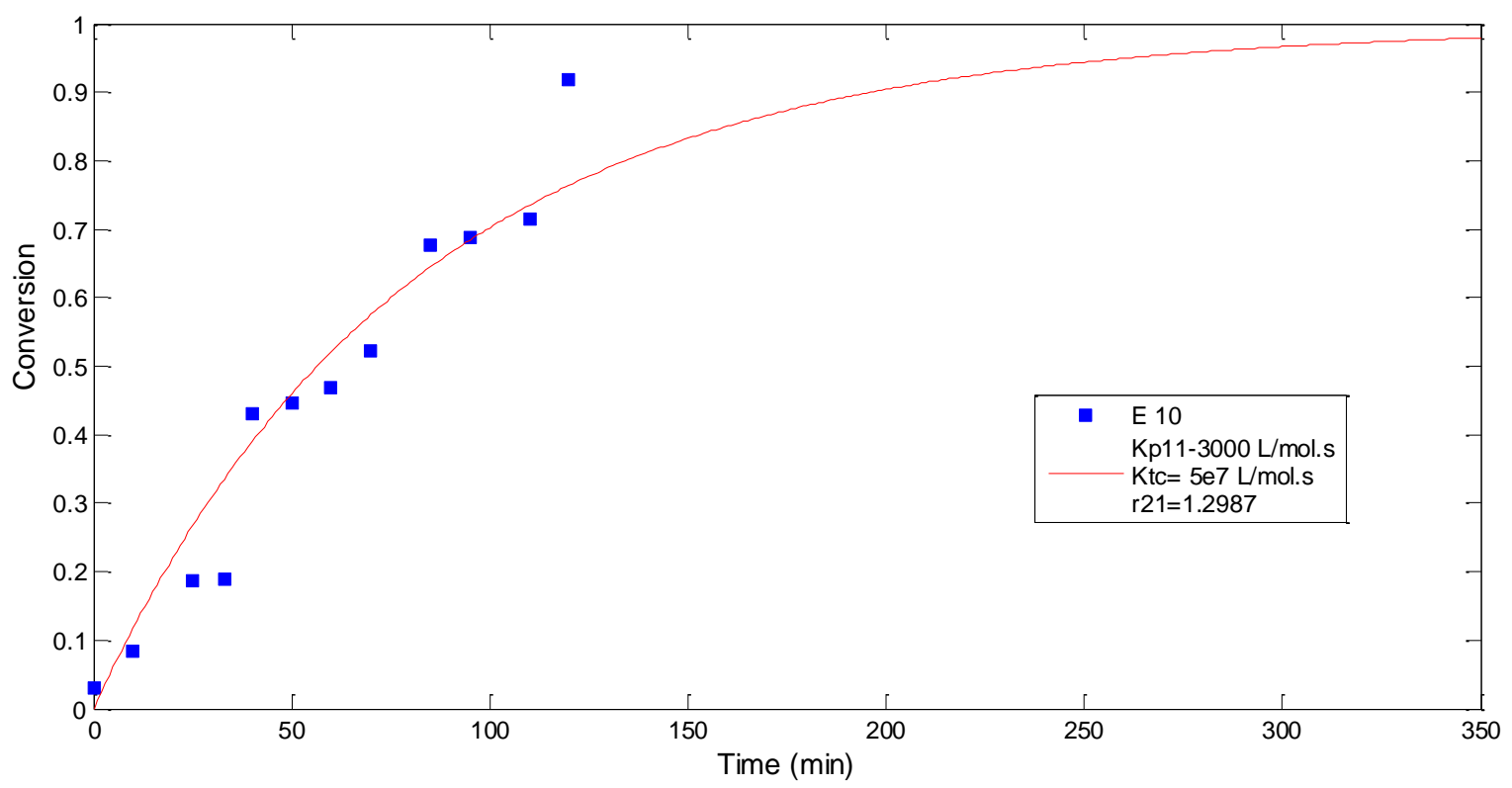

Figure 35. Conversion-time dependence of the rate coefficient homopropagation of acrylic acid (Kp11) influence of crosslinkers TMPTA $60^{\circ} \mathrm{C}$

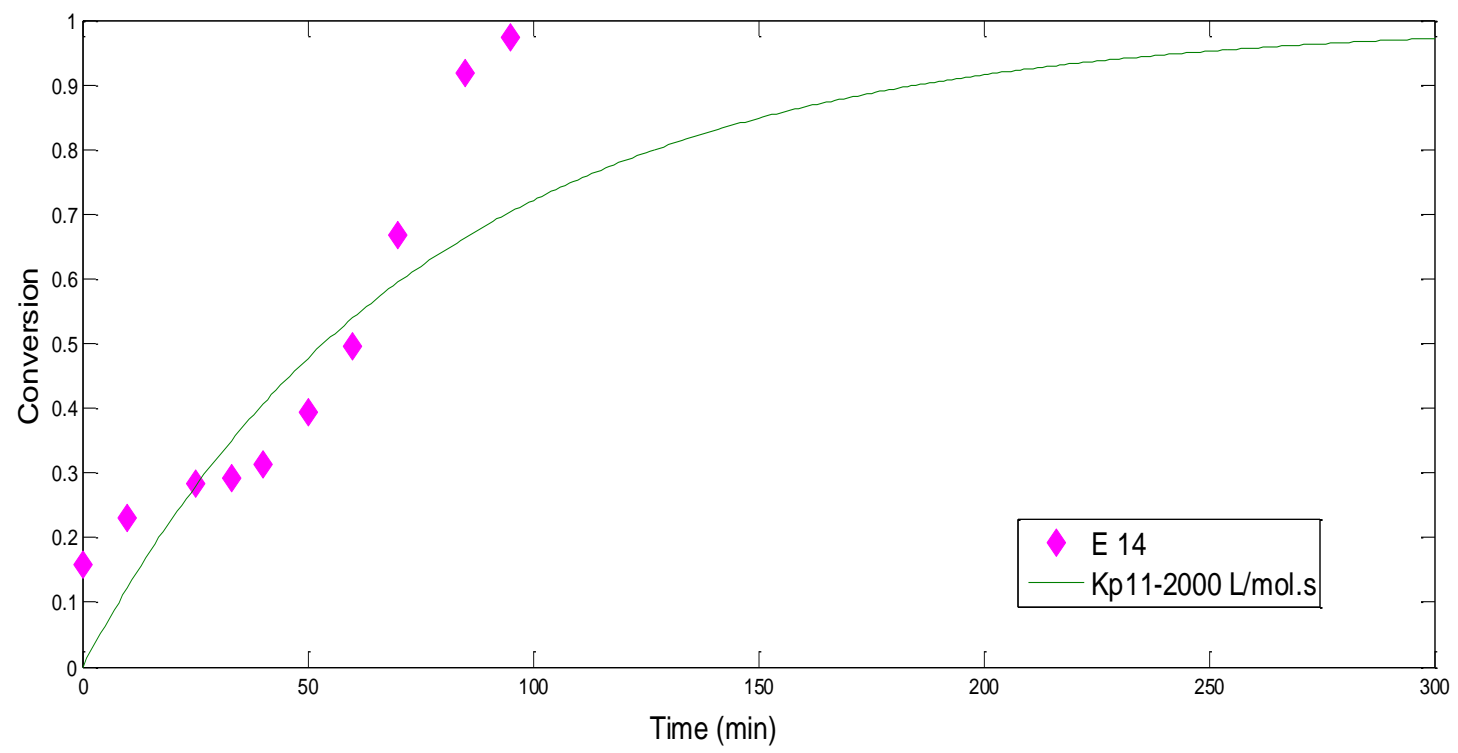

Figure 36. Conversion-time rate different values of the coefficient homopropagation of acrylic acid (Kp11) Influence of crosslinkers EGDMA 


\subsubsection{Run with low concentration of Sodium persulfate}

Figure 37 compares the experimental results and simulation of run E-6, in which a lower concentration of sodium persulfate $(0.03 w \mathrm{w} \%)$ was employed. Based on the experimental data, the induction time used in the simulation was assumed to be 30 minutes. The adjusted value of $\mathrm{K}_{\mathrm{P} 11}$ was $1700 \mathrm{~L} /(\mathrm{mol} . \mathrm{s})$.

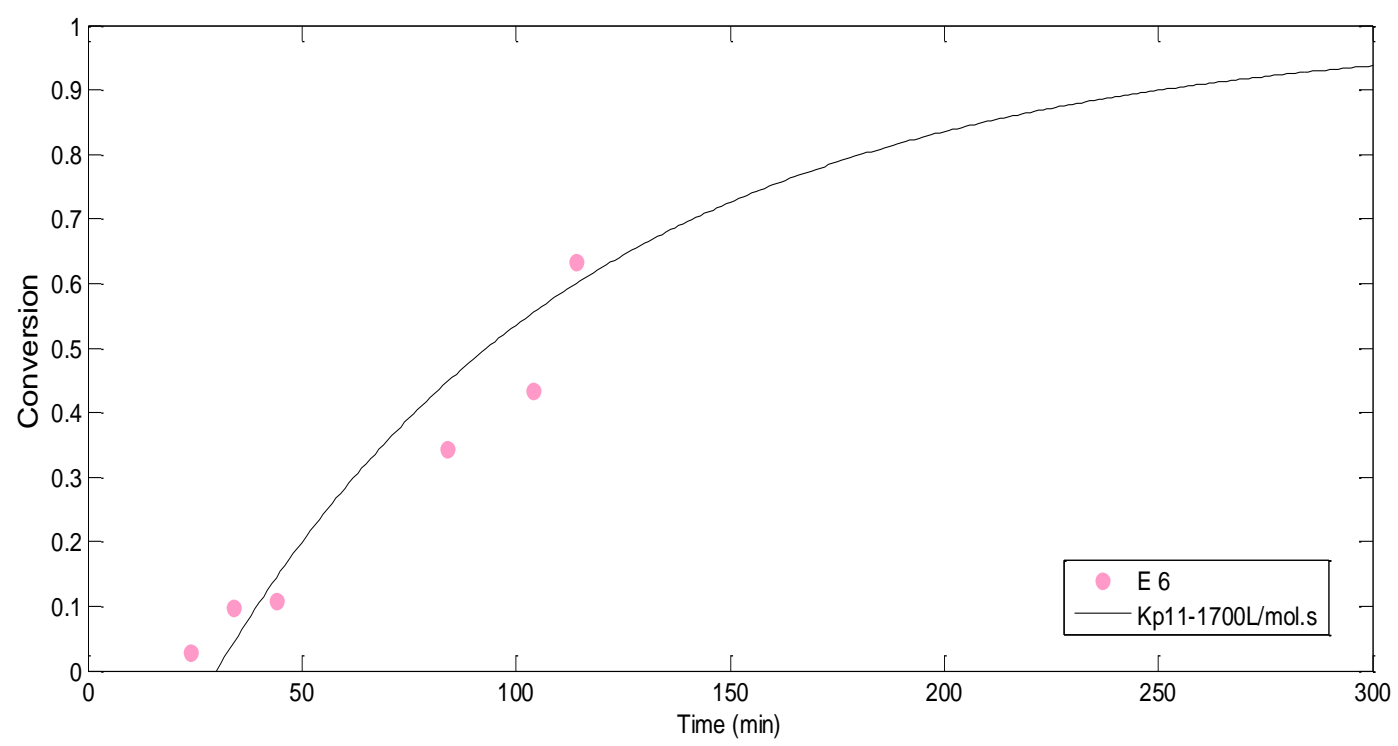

Figure 37. Conversion-time different values of the rate coefficient for the homopropagation of acrylic acid $\left(\mathrm{Kp}_{11}\right)$ influence low initiator concentration of sodium persulfate

\subsubsection{Run with long feeding time of the aqueous phase}

Figure 38 compares the experimental and simulated results for Run E8 that was performed after a longer feeding time (60 minutes, before the time zero). No induction time was considered in the simulation and a value of $K_{P 11}=1000$ L/(mol.s) was adjusted.

The purpose of this experiment was to analyze if there occurs kinetic changes in relation to feeding from the aqueous phase and how it could affect the reaction medium. 


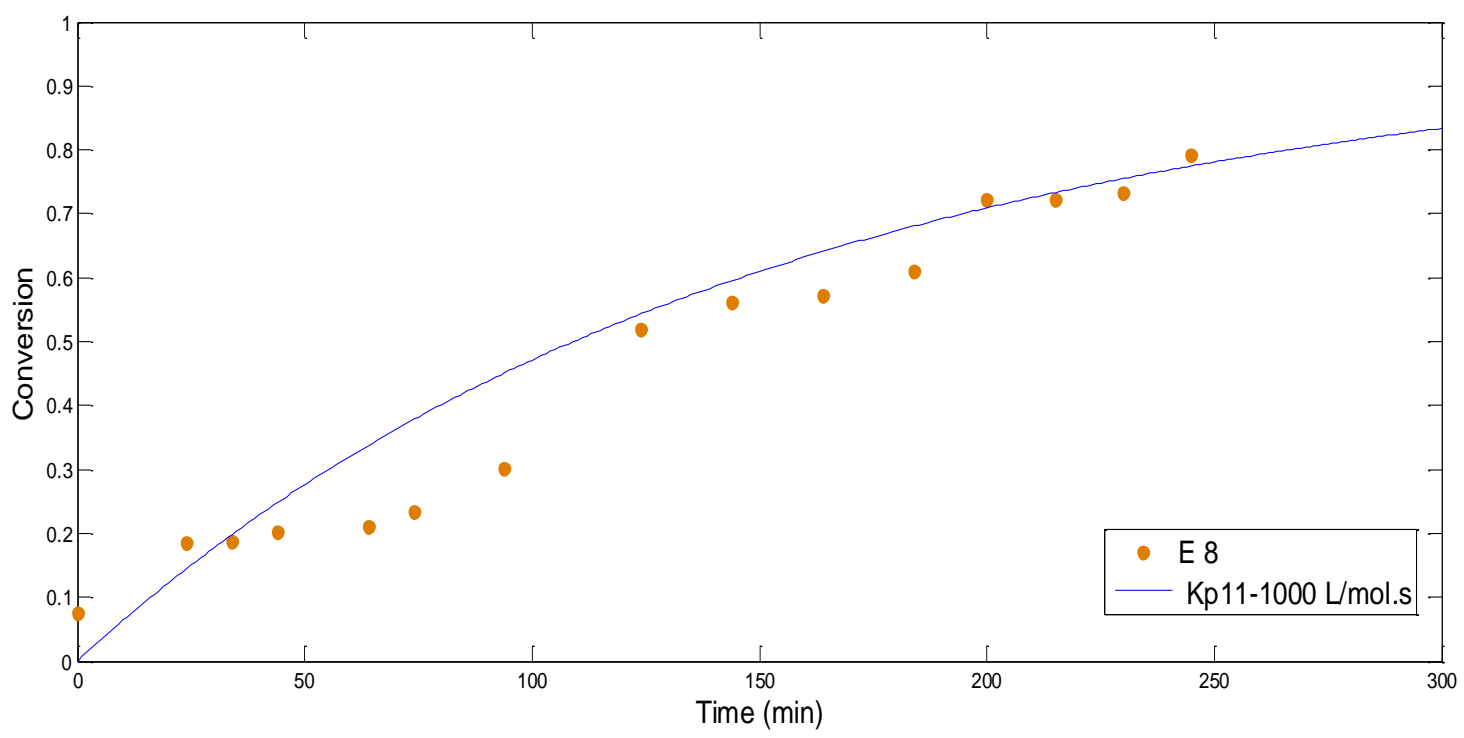

Figure 38. Conversion-time different values of the rate coefficient homopropagation of acrylic acid (Kp11)influence on feed flow rate time

\subsubsection{Run with low concentration of acrylic acid}

The experiment E9 was conducted at low initial concentration of monomer. Figure 38 shows the experimental and simulation results. An unexpectedly long induction time was observed (a value of 154 minutes was assumed to fit the model to this run) and a the adjusted value of $\mathrm{K}_{\mathrm{P} 11}$ was $3000 \mathrm{~L} /(\mathrm{mol} . \mathrm{s})$.

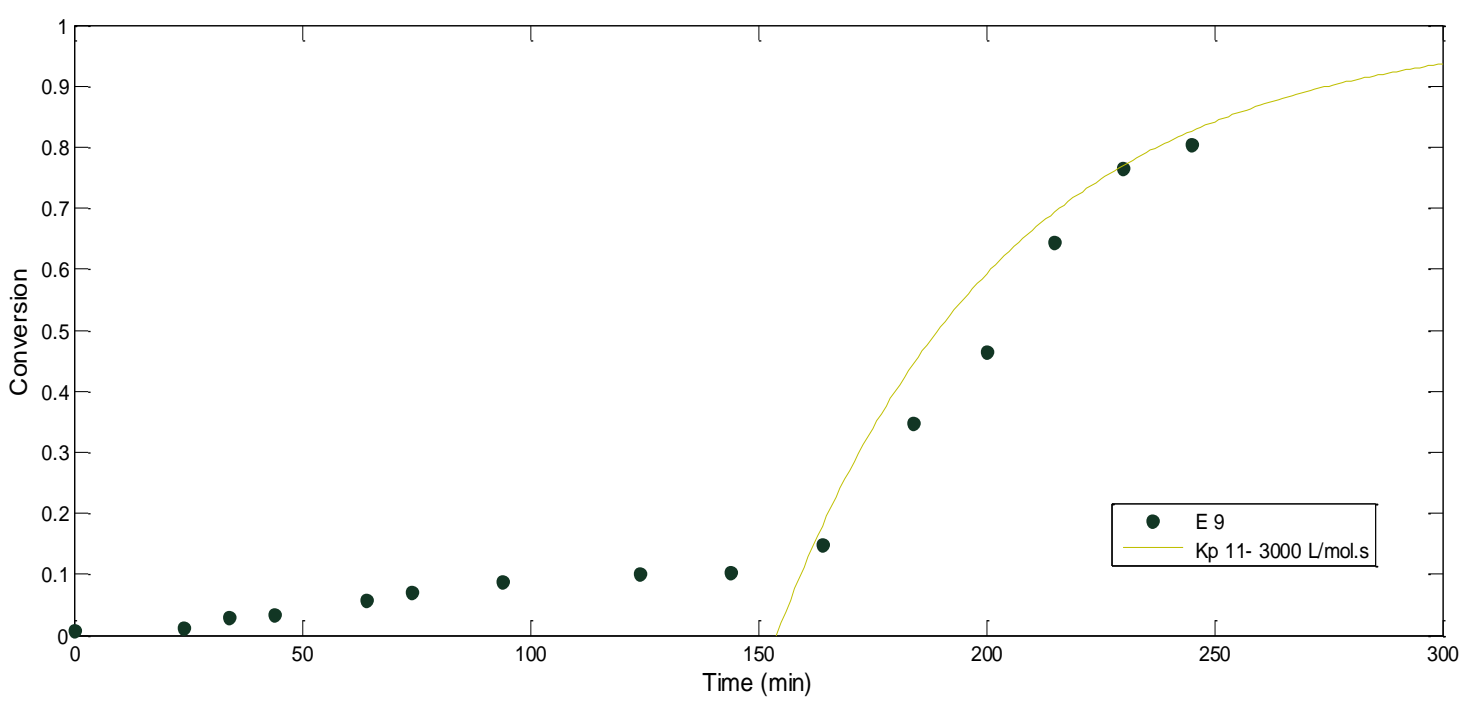

Figure 39. Conversion-time different values of the rate coefficient for the homopropagation of acrylic acid $\left(\mathrm{Kp}_{11}\right)$ different acrylic acid concentration at $60^{\circ} \mathrm{C}$ 


\subsubsection{Deoxygenation period}

Experiments E15, E16, E17 and E18, all conducted with a low concentration of initiator, are shown in Figures 40 to 43 . The commercial monomer containing $200 \mathrm{ppm}$ of MEHQ was previously purified by distillation, aerated by bubbling air for 60 minutes, and then deoxygenated by bubbling nitrogen for different deoxigenation times $(0,15,30$ and 60 minutes).

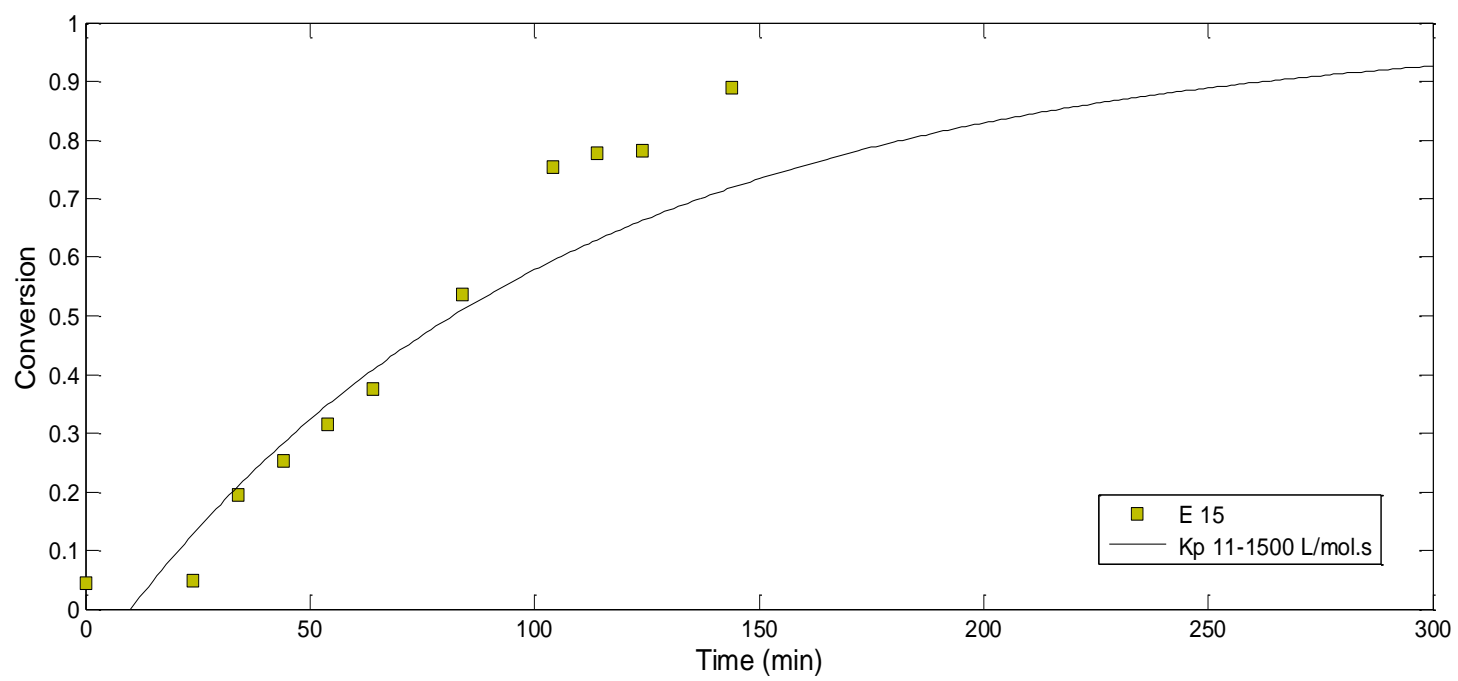

Figure 40. Conversion-time dependence rate coefficient homopropagation of acrylic acid $\left(\mathrm{Kp}_{11}\right), 1500 \mathrm{~L} / \mathrm{mol} . \mathrm{s}$ Polymerization rate and inhibition time as a function of deoxygenation condition- No Deox

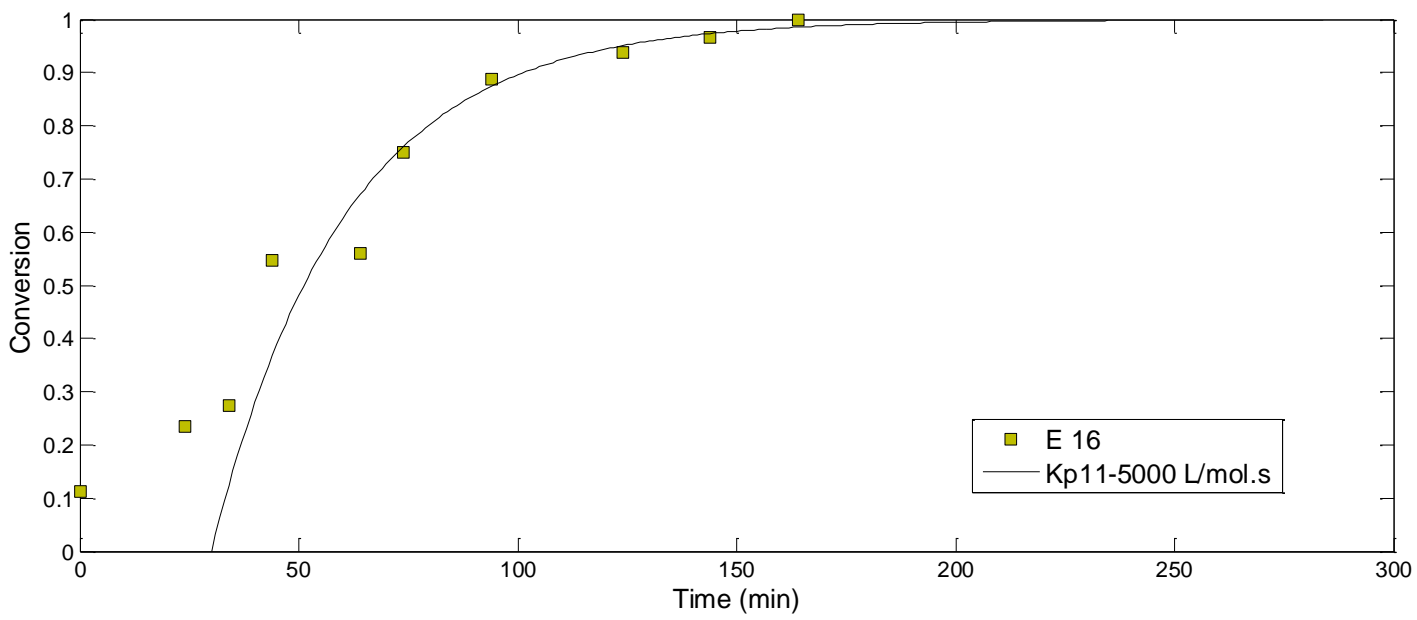

Figure 41. Conversion-time dependence of rate coefficient homopropagation of acrylic acid (Kp11) 5000 $\mathrm{L} / \mathrm{mol}$.s -Polymerization rate and inhibition time as a function of $15 \mathrm{~min}$ of deoxygenation condition 
The values of $K_{P 11}$ adjusted varied within a short range, 1500 to 5000 $\mathrm{L} /(\mathrm{mol} . \mathrm{s})$. Probably a unique value would be able to fit reasonably the data measured in all four runs.

Impurities that come with the nitrogen can affect the normal process of the polymerization reaction in inverse suspension, showing, in some reactions, low propagation constant values.

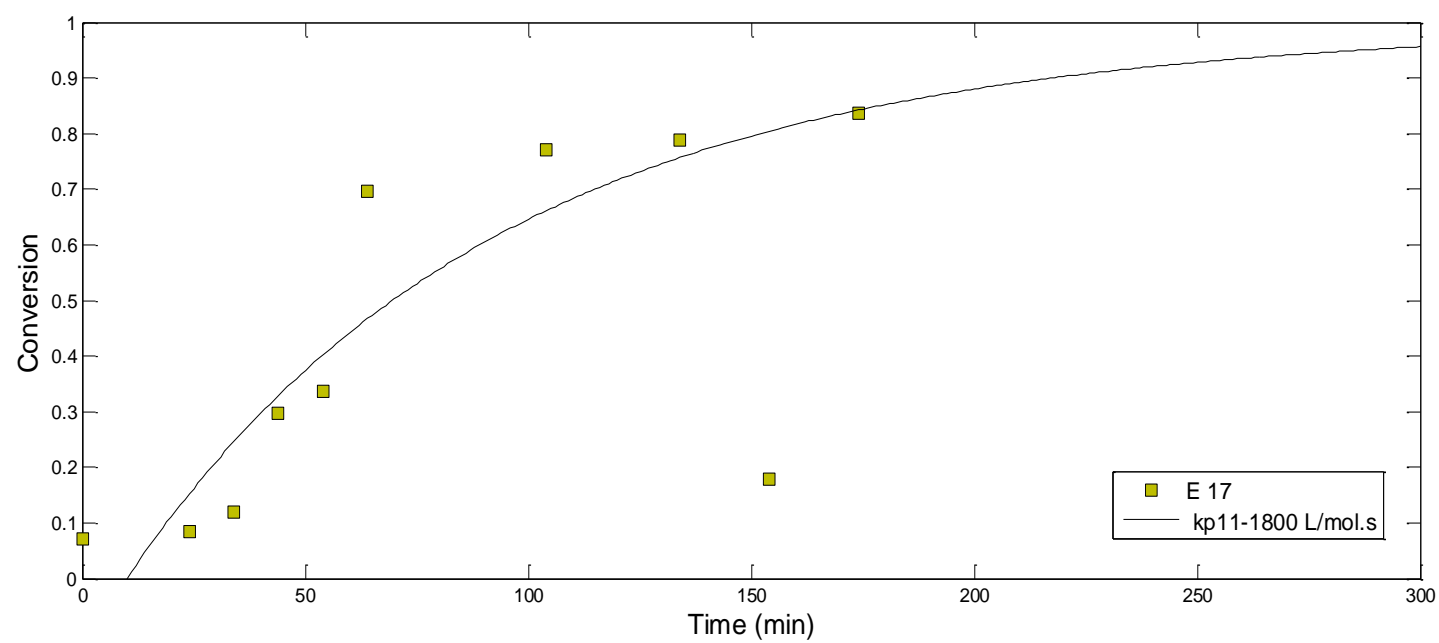

Figure 42. Conversion-time rate coefficient homopropagation of acrylic acid (Kp11) $2000 \mathrm{~L} / \mathrm{mol} . \mathrm{s}$ Polymerization rate and inhibition time as a function of 30 min of deoxygenation condition

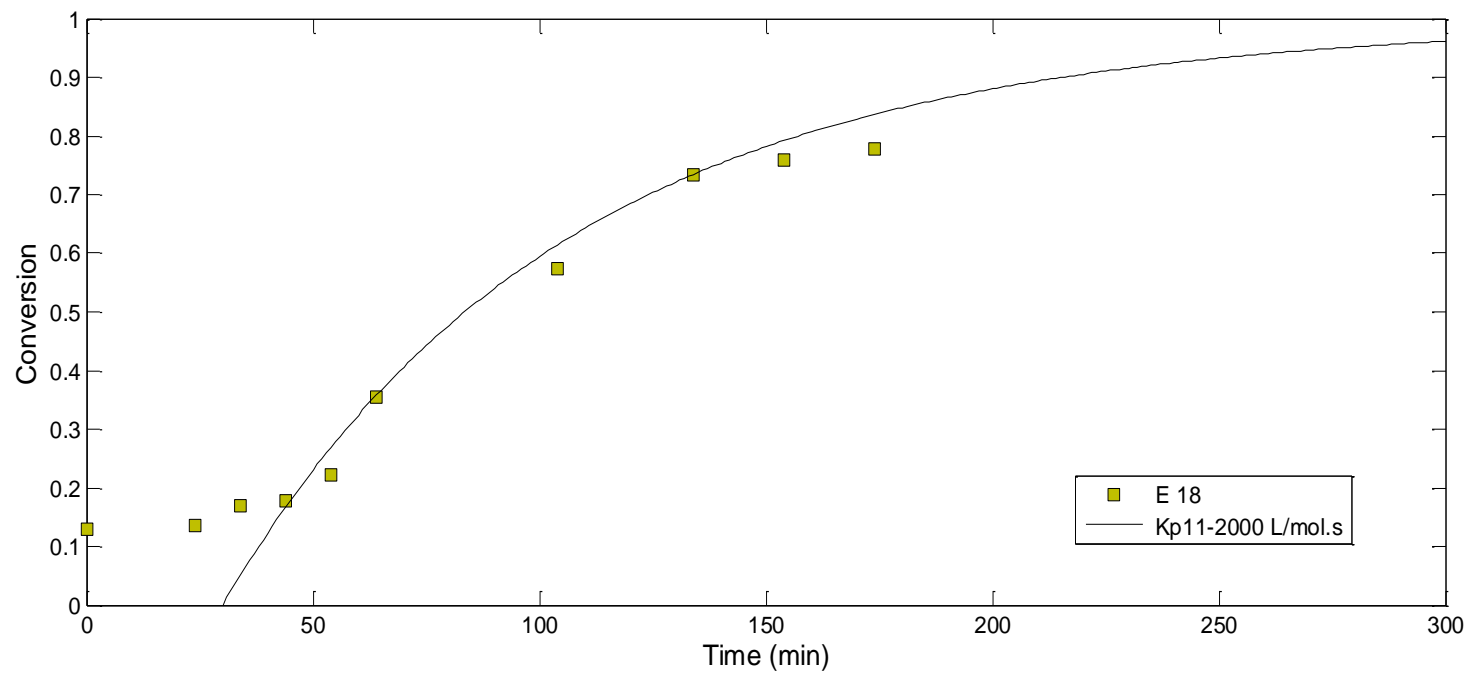

Figure 43. Conversion-time dependence of the rate coefficient homopropagation of acrylic acid (Kp11) 2000 $\mathrm{L} / \mathrm{mol}$.s -Polymerization rate and inhibition time as a function of $60 \mathrm{~min}$ of deoxygenation condition 


\subsection{Analysis of the dynamics of gelation by the numerical fractionation technique}

In this section, a comparison is presented between the gel fraction profile obtained from the model developed in this work.

The reactivity ratios for pendant double bond polymerization was evaluated in these simulations, as shown from Figures 44 to 46 , to observe its effect on the model curves of gel fraction.

Also, the effect of the number of generations considered in the simulations was also studies. The number of generations is a parameter of the numerical fractionation technique by Teymour (1994). In principle, the higher the number of generations considered, the better the approximation of the model to calculate the gel fraction. On the other hand, the increase of number of generations causes an increase in the computational effort to solve the model. Therefore, the number of generations should be chosen as the lowest value to which the further increase does not change significantly the model responses.

In the simulations, it was observed that the computer program does not provide good results when more than 5 generations is chosen. This limitation is probably related to the use of an ODE solver for nonstiff systems (ode45) in the MATLAB program. When the number of generations was increased, the stiffness of the ODE system increased and the solver failed.

The effect of the number of generations on the simulation results is shown in Figure 44. It can be seen that the differences in the simulated value of gel fraction decrease as the number of generations increases. The difference becomes negligible for simulations with 4 and 5 generations, indicating the use of 5 generations is sufficient to the correct prediction of the gel fraction. 


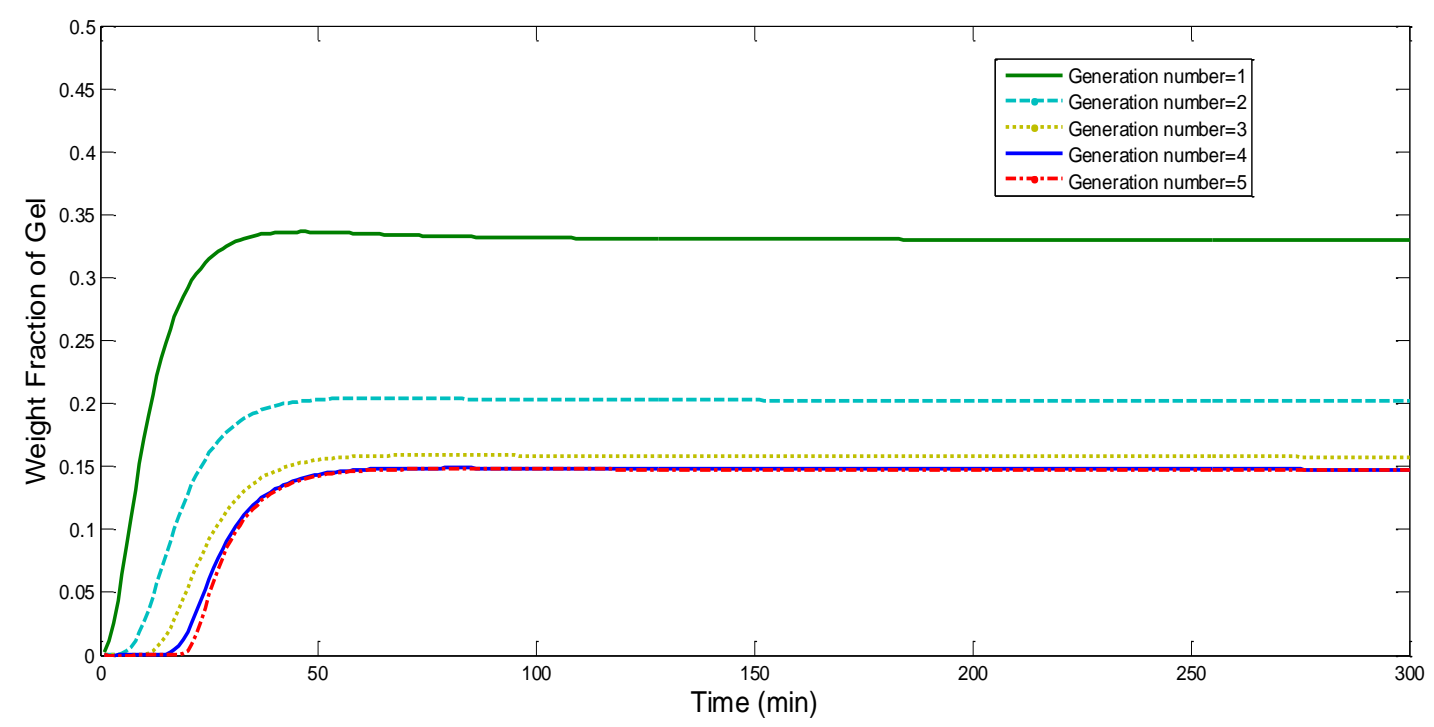

Figure 44. Evolution of the gel fraction calculated using different numbers of generations $(n=1,23,4$ and 5).

The effect of the reactivity of the PDBs on the dynamics of gel formation is shown in Figures 45 and 46 . These simulations were performed with 5 generations. Under these conditions, the reactivity of pendant double bonds ( $\left.r_{\text {PDB }}\right)$ has an effect on the dynamics of gel formation. As expected, lower gel content is predicted if the reactivity of PDBs is much lower than for the double bonds of the crosslinker ( $r_{\mathrm{PDB}}$ 0.3). The gel content -increases with the increase of the value of this parameter $\left(r_{\mathrm{PDB}}\right)$, as shown in Figure 46 (in comparision with Figure 45 ). Due to the low content of crosslinker in the polymerization system, the overall monomer conversion (not shown) is almost insensitive to this parameter. The reactivity of the all the three double bonds of TMPTA to react with acrylic acid would be expected to, but once incorporated into the polymer network, the reactivity of the other two remaining double bonds are assumed to be lower. 


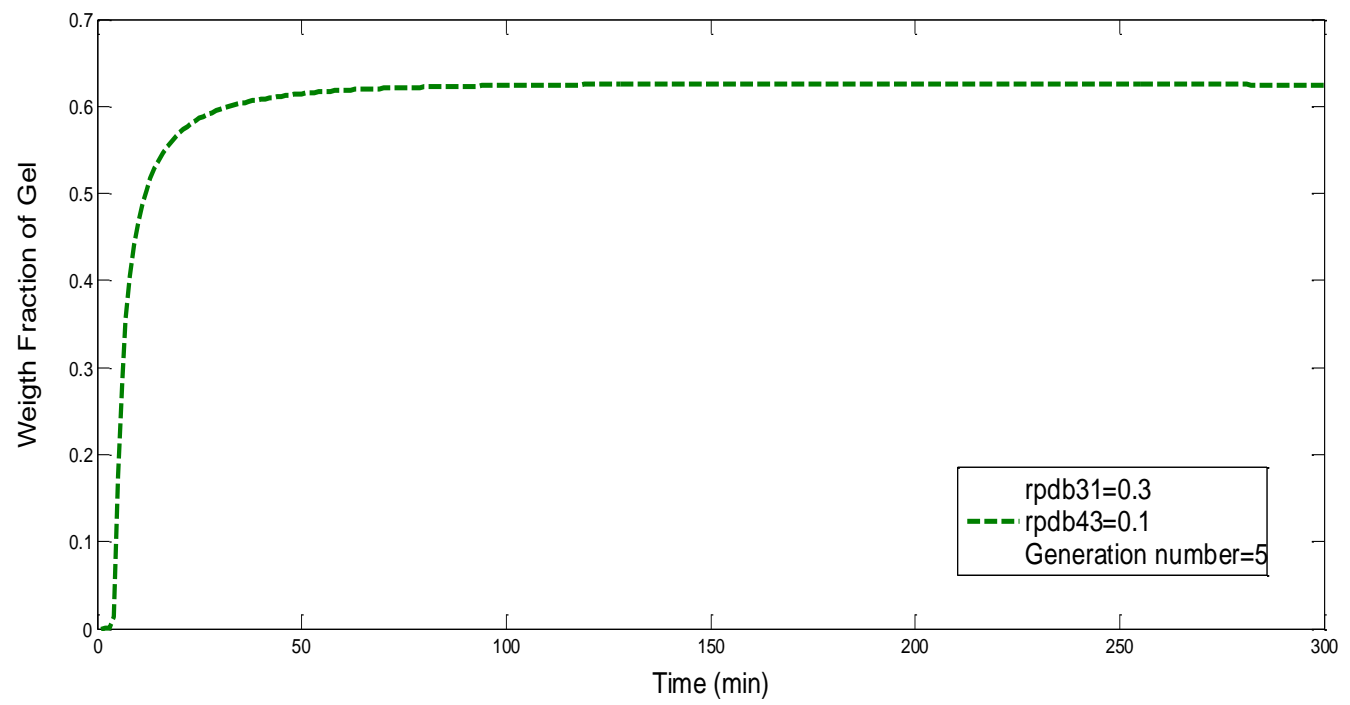

Figure 45. Evolution of the gel fraction calculated with numerical fractionation technique with 5 generations using different reactivity ratios of PDBs

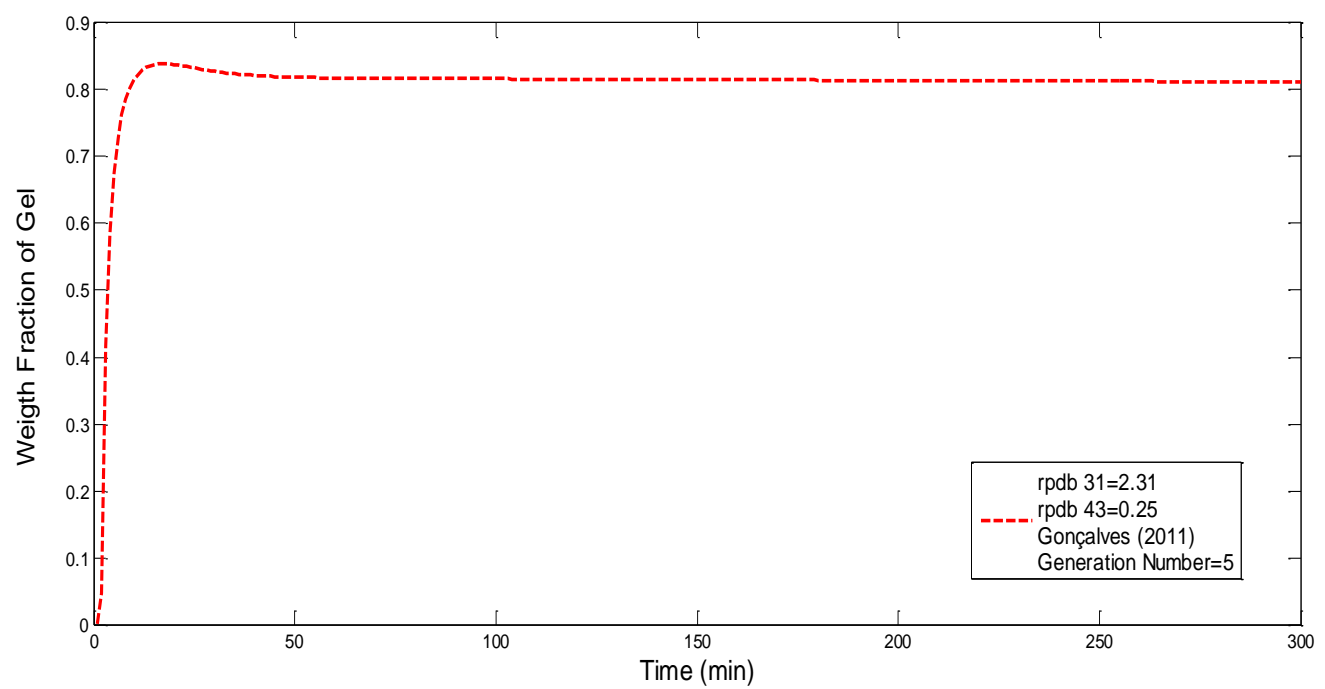

Figure 46. Evolution of the gel fraction calculated with numerical fractionation technique with 5 generations using different reactivity ratios of PDBs 


\subsection{General discussions}

This work was proposed to evaluate some key variables of the process of inverse suspensions polymerization of acrylic acid (and crosslinkers) to produce hydrogel. Experiments were carried out in laboratory-scale reactor and monomer conversion and gel fraction were measured as a function of time during the polymerization. The effects of initiator concentration, feed flow rate, crosslinker concentration and monomer concentration on these results were evaluated.

These possible scenarios may account for some sources of different induction period, uncertainties at different stages of the experimentation and the analytical testing methods used for calculation of polymerization rate and inhibition time, as function of deoxygenation conditions, which need to be considered. The induction period varied a lot in the different runs, reflecting either the presence of $\mathrm{MEHQ}$ inhibitor in the commercial monomers (different concentrations due to different lots of monomer, or different storage time of the same lot) or the effect of the dissolved oxygen in the aqueous phase. A limited number of experiments were performed with purified acrylic acid monomer (which was distilled to remove the inhibitor) trying to assess the effect of dissolved oxygen by changing the deoxygenation time.

Three types of crosslinkers, namely trimetylolpropane triacrylate (TMPTA), tetraallyloxyethane (TAO) and ethylene glycol dimethacrylate (EGDMA), were employed in the experiments to compare their effectiveness as a comonomer/ crosslinker. This kind of crosslinking agent is typically used for generating highlycrosslinked polymer structures, thus increasing polymer toughness, modulus and solvent resistance. An increase in the comonomer functionality dramatically decreases the general mobility of monomer functional group in the medium during polymerization due to the earlier formation of a crosslinked network hydrogel even at very low conversions. For example, trimethylol propane triacrylate, a trifunctional monomer, is less sensitive to oxygen inhibition than the bifunctional comonomer. Monomer structure may also be a significant factor in reducing oxygen inhibition especially when monomers have abstractable hydrogen such as vinyl ether groups, which are among the most common functional groups. 
The conversion profiles over all conditions and modes of operation are generally well represented by a model that uses, after the adjustment of the propagation rate constant for acrylic acid $\left(\mathrm{K}_{\mathrm{p} 11}\right)$ and imposing in the simulations an initial period of induction caused by the inhibition. This induction time was obtained from the experimental observation. The quantitative prediction of the effect of the inhibitor on the simulated results would require to change the kinetic model in order to include explicitly the inhibition reactions, which is easy to do, but would require the estimation of the involved kinetic parameters of the inhibition process. This would require additional measurements in experiments specially designed for this purpose. 


\section{CHAPTER VI}

\section{CONCLUSIONS AND SUGGESTIONS}

\subsection{Conclusions}

In this topic the main conclusions and contributions are presented:

In the present work, experimental studies and mathematical model were developed for the homopolymerization and copolymerization of acrylic acid in an inverse suspension polymerization process, for the determination of the monomer conversion and gel fraction. The effects of temperature, monomer concentration, concentration and type of of the comonomer/crosslinker, concentration of the initiator, and feeding time of the aqueous phase were studied.

The results contribute to increase the database of experimental data of this process and to test the mathematical model, thus contribute to improve the general knowledge on this complex process.

The experimental measurements of monomer conversion by gravimetry presented errors of about $15 \%$. This is still acceptable, but is higher than the usual uncertainties of this method. These errors are related to the size of the samples and to the formation of dry skin in the samples during drying. This reinforces the special care required in conversion measurements in gelling polymerization systems.

For some runs, gel fraction was measured by extraction with solvent (water), which is also a delicate measurement technique subject to errors related to the incomplete extraction and with the propagation of the weighting errors due to very small samples involved. The obtained results for gel fraction were sound and acceptable, indicating the employed extraction method was reliable.

Some polymerization reactions showed extensive induction time, which was caused by the presence of dissolved oxygen and the content of MEHQ inhibitor in 
the commercial-grade monomer. Some experiments were performed with distilled monomer and by changing the dissolved oxygen in a systematic way, yet the dissolved oxygen was not directly measured. These results show that both oxygen and MEHQ affect the results.

A mathematical model for the process was developed based on the previous models reported in the literature, in special that of Gonçalves et al. (2011). The model predicted the time history of monomer conversion and gel fraction to be compared with the data collected in the experiments.

The model does not account for explicitly the inhibition reactions, so it is not capable of predict the induction time observed experimentally. In order to account for the induction period in the simulated curves, the zero time of the simulated curves were shifted towards higher times by adding a specified value of induction time directly estimated from the experimental data.

After this induction time, the simulated curves were fitted to the experimental data by adjusting the value of the kinetic constants of propagation of acrylic acid (KP11), in a similar way performed in the literature (Arriola et al., 1996; Gonçalves et al., 2011). The obtained values of this rate constant increases with temperature, as expected.

The gel fraction was calculated in the model by using the numerical fractionation technique. The number of generations was studied and determined that 5 generations were sufficient for the gel fraction calculation.

The comparison of gel fraction predictions with the experimental data shows that additional fitting should be necessary, because the model is predicting that the gel point occurs much after the gel point started in practice.

In summary, this research provided new experimental data and a better understanding of some of the variables affecting the inverse suspension polymerization of acrylic acid and TMPTA. The mathematical model developed for this process is suitable to represent the main features of this system, was partially 
adjusted to the monomer conversion data and should be better fitted to the gel fraction by adjusting additional kinetic parameters.

\subsection{Suggestions for Future Work}

The following suggestions can be recommended to future works in this topic:

\section{- Improve the mathematical model to account for the effect of inhibitors}

The model could be improved by including explicitly the kinetics of the inhibition by both dissolved oxygen and MEHQ. The work of Li and Schork (2006) would provide a general basis to change the kinetic model and balance equations accordingly. Specific experiments (see, e.g., Cutie et al., 1997) could be designed to assess these effects and to allow for estimation of the unknown parameters.

- Study the kinetics with DSC and FTIR, and measurement of $\mathrm{pH}$ to controlling to $K_{P}$

Use of DSC is appropriate for recording the heat production of chemical and physical process and for obtaining data changes with time to follow the kinetics of the reaction. This allows determining the dependence of the polymerization rate on various reaction parameters. And also use the FTIR in-line monitoring technique, in order to determinate the monomer conversion and validate with the gravimetric analysis, giving a better perspective of what is happening inside the reactor

- Study the hydrogel performance in controlled releasing of drugs continuing the research to characterize the controlled release of pharmaceutical products (like insulin) from the hydrogel matrix, performing and simulating changes of $\mathrm{pH}$, and identify which of the samples of hydrogels is the most suitable for this process 
- Neutralization of Acrylic acid, aqueous solution of acrylic acid salts obtain trough the neutralization of $\mathrm{AA}$, with a base such as $\mathrm{NaOH}$, are also usually consider in SAP production, improving condition medium reaction due to $\mathrm{pH}$ control, reach a higher conversion and also gel fraction results.

- SEM micrographic of particles The particle size distributions of hydrogels, is one of the topics of great importance in processes make by inverse suspension polymerization and the dependence of absorption, studying the characteristics on particle size using the MEV technique and determinate another variable such as the salinity of the water on absorption effects, and develop a model to accompany this type of research.

- Swelling of crosslinkers is one of the important physical properties of hydrogels and depend on the precise structure of the polymer network, of key importance is equilibrium swelling capacity. The effect of different crosslinkers (e.g., TMPTA, TAO and EGDMA) on the swelling capacity of the hydrogel. Molecular theories of rubber elasticity describe with varying accuracy the relationship between the molecular structure of a crosslinked polymer and the amount of swelling. Crosslink density can be determined by the two methods, modulus and swelling measurements.

- NIR Infrared spectroscopy prediction of the evolution of monomer concentration, conversion and average particle diameter in acrylic acid in inverse suspension polymerization using different kind of initiators such as AIBN, benzoyl peroxide, hydroperoxide. Multivariate partial least squares calibration models can be developed to relate NIR spectra collected by the immersion sensor with online conversion and polymer particle size data, values predicted by the NIR calibration models and these latter were also able to detect different types of operational disturbances. 


\section{REFERENCES}

AGUIAR, L. G. et al. Development of Cyclic Propagation Kinetics for Modeling the Nitroxide-mediated Radical Copolymerization of Styrene-Divinylbenzene Macromolecular Reaction Engineering, v. 8, n. 4, p. 282-294, 2014.

AHMED, E. M. Hydrogel: Preparation, characterization, and applications. Journal of Advanced Research, 2013.

ARRIOLA, D. J. et al. Crosslinker reactivity and the structure of superabsorbent gels. Journal of Applied Polymer Science, v. 63, n. 4, p. 439-451, 1997.

BOWMAN, C. N.; PEPPAS, N. A. A kinetic gelation method for the simulation of free-radical polymerizations. Chemical Engineering Science, v. 47, n. 6, p. 14111419, 1992.

BUNYAKAN, C., \& Hunkeler, D. Precipitation polymerization of acrylic acid in toluene. I: synthesis, characterization and kinetics. Polymer, 40(23), 6213-6224. (1999).

BUNYAKAN, C., \& Hunkeler, D. Precipitation polymerization of acrylic acid in toluene. II: synthesis, characterization and kinetics. Polymer, 40(23), 6213-6224. (1999).

CHOUDHARY, M. S. Inverse Suspension Polymerization of Partially Neutralized and Lightly Cross-Linked Acrylic Acid: Effect of Reaction Parameters. Macromolecular Symposia, v. 277, n. 1, p. 171-176, 1 fev. 2009.

COSTA, M. R.; DIAS, R. Prediction of sol fraction and average molecular weights after gelation for non-linear free radical polymerizations using a kinetic approach. 2003. 
CUTIÉ, S. S. et al. The effects of MEHQ on the polymerization of acrylic acid in the preparation of superabsorbent gels. Journal of Applied Polymer Science, v. 64, n. 3, p. 577-589, 18 abr. 1997.

CUTIÉ, S. S. et al. Acrylic acid polymerization kinetics. Journal of Polymer Science Part B: Polymer Physics, v. 35, n. 13, p. 2029-2047, 30 set. 1997.

GONÇALVES, M. et al. Modeling studies on the synthesis of superabsorbent hydrogels using population balance equations. 2011.

GUPTA, P.; VERMANI, K.; GARG, S. Hydrogels: from controlled release to $\mathrm{pH}-$ responsive drug delivery. Drug Discovery Today, v. 7, n. 10, p. 569-579, 15 maio. 2002.

HARRISSON, S.; MACKENZIE, S. R.; HADDLETON, D. M. Pulsed Laser Polymerization in an lonic Liquid: Strong Solvent Effects on Propagation and Termination of Methyl Methacrylate. Macromolecules, v. 36, n. 14, p. 5072-5075, 1 jul. 2003.

HENNINK, W. E.; NOSTRUM, C. F. VAN. Novel crosslinking methods to design hydrogels. Advanced Drug Delivery Reviews, v. 64, p. 223-236, dez. 2012.

HENTON, D. E.; POWELL, C.; REIM, R. E. The decomposition of sodium persulfate in the presence of acrylic acid. Journal of Applied Polymer Science, v. 64, n. 3, p. 591-600, 1997.

H. OMIDIAN, M. J. Z.-M. Polymerization of sodium acrylate in inverse-suspension stabilized by sorbitan fatty esters. European Polymer Journal, v. 39, n. 5, p. 10131018, 2003. 
HUSSAIN, Y. A.; LIU, T.; ROBERTS, G. W. Synthesis of Cross-Linked, Partially Neutralized Poly(Acrylic Acid) by Suspension Polymerization in Supercritical Carbon Dioxide. Industrial \& Engineering Chemistry Research, v. 51, n. 35, p. 11401-11408, 5 set. 2012.

JAHANZAD, F.; SAJJADI, S.; BROOKS, B. W. Characteristic intervals in suspension polymerisation reactors: An experimental and modelling study. Chemical Engineering Science, v. 60, n. 20, p. 5574-5589, out. 2005a.

JAHANZAD, F.; SAJJADI, S.; BROOKS, B. W. Characteristic intervals in suspension polymerisation reactors: An experimental and modelling study. Chemical Engineering Science, v. 60, n. 20, p. 5574-5589, out. 2005b.

KALFAS, G.; YUAN, H.; RAY, W. H. Modeling and experimental studies of aqueous suspension polymerization processes. 2. Experiments in batch reactors. Industrial \& Engineering Chemistry Research, v. 32, n. 9, p. 1831-1838, 1 set. 1993.

KIZILEL, S. et al. Mathematical Model for Vinyl-Divinyl Polymerization. Macromolecular Reaction Engineering, v. 1, n. 6, p. 587-603, 20 nov. 2007.

KUCHTA, F.-D.; HERK, A. M. VAN; GERMAN, A. L. Propagation Kinetics of Acrylic and Methacrylic Acid in Water and Organic Solvents Studied by Pulsed-Laser Polymerization. Macromolecules, v. 33, n. 10, p. 3641-3649, 1 maio. 2000.

LACÍK, I.; BEUERMANN, S.; BUBACK, M. PLP-SEC Study into Free-Radical Propagation Rate of Nonionized Acrylic Acid in Aqueous Solution. Macromolecules, v. 36, n. 25, p. 9355-9363, 1 dez. 2003. 
LIN, H.-R. Solution polymerization of acrylamide using potassium persulfate as an initiator: kinetic studies, temperature and $\mathrm{pH}$ dependence. European Polymer Journal, v. 37, n. 7, p. 1507-1510, jul. 2001.

LI, R.; SCHORK, F. J. Modeling of the Inhibition Mechanism of Acrylic Acid Polymerization. Industrial \& Engineering Chemistry Research, v. 45, n. 9, p. 30013008, 1 abr. 2006.

LOISEAU, J. et al. Synthesis and Characterization of Poly(acrylic acid) Produced by RAFT Polymerization. Application as a Very Efficient Dispersant of $\mathrm{CaCO}$, Kaolin, and TiO2. Macromolecules, v. 36, n. 9, p. 3066-3077, 2003.

LORBER, N., Pavageau, B., \& Mignard, E. Droplet-based millifluidics as a new miniaturized tool to investigate polymerization reactions.Macromolecules, 43(13), 5524-5529. (2010).

MACHADO, F.; LIMA, E. L.; PINTO, J. C. A review on suspension polymerization processes. Polímeros, v. 17, n. 2, p. 166-179, jun. 2007a.

MACHADO, F.; LIMA, E. L.; PINTO, J. C. Dynamic optimization of semibatch vinyl acetate/acrylic acid suspension copolymerizations. Polymer Engineering \& Science, v. 50, n. 4, p. 697-708, 1 abr. 2010a.

MACHADO, F.; LIMA, E. L.; PINTO, J. C. Dynamic optimization of semibatch vinyl acetate/acrylic acid suspension copolymerizations. Polymer Engineering \& Science, v. 50, n. 4, p. 697-708, 1 abr. 2010b.

MARCELO KAMINSKI LENZI, F. M. S. Semibatch styrene suspension polymerization processes. Journal of Applied Polymer Science, v. 89, n. 11, p. 3021 - 3038, 2003. 
MAYOUX, C. et al. Inverse suspension polymerization of sodium acrylate: Synthesis and characterization. Journal of Applied Polymer Science, v. 77, n. 12, p. $2621-2630,19$ set. 2000.

MURLI, C.; SONG, Y. Pressure-Induced Polymerization of Acrylic Acid: A Raman Spectroscopic Study. The Journal of Physical Chemistry B, v. 114, n. 30, p. 97449750, 2010.

$\mathrm{NI}, \mathrm{X}$. et al. Suspension polymerization of acrylamide in an oscillatory baffled reactor: From drops to particles. AIChE Journal, v. 47, n. 8, p. 1746-1757, 1 ago. 2001.

OKAY, O. Gel growth in free radical crosslinking copolymerization: Effect of inactive gel radicals. Macromolecular Theory and Simulations, v. 9, n. 6, p. 354361,1 jul. 2000b.

OMIDIAN, H.; HASHEMI, S. A.; SAMMES, P. G.; MELDRUM, I. G. Modified acrylic-based superabsorbent polymers. Effect of temperature and initiator concentration. Polymer, v. 39, n. 15, p. 3459-3466, jul. 1998.

OMIDIAN, H. et al. Modified acrylic-based superabsorbent polymers (dependence on particle size and salinity). Polymer, v. 40, n. 7, p. 1753-1761, mar. 1999.

PARK, H.; ROBINSON, J. R. Mechanisms of mucoadhesion of poly(acrylic acid) hydrogels. Pharmaceutical Research, v. 4, n. 6, p. 457-464, dez. 1987.

QIU, L., Wang, K., Zhu, S., Lu, Y., \& Luo, G. Kinetics study of acrylic acid polymerization with a microreactor platform. Chemical Engineering Journal, 284, 233-239. (2006). 
RINTOUL, I.; WANDREY, C. Polymerization of ionic monomers in polar solvents: kinetics and mechanism of the free radical copolymerization of acrylamide/acrylic acid. Polymer, v. 46, n. 13, p. 4525-4532, 17 jun. 2005.

SILVA, F. M.; LIMA, E. L.; PINTO, J. C. Control of the Copolymer Composition in Suspension Copolymerization Reactions. Industrial \& Engineering Chemistry Research, v. 43, n. 23, p. 7312-7323, 1 nov. 2004.

SOUZA, E. F. Estudo experimental e modelação matemática do processo de produção de hidrogéis de poli(ácido acrílico). 2013.

TEYMOUR, F.; CAMPBELL, J. D. Analysis of the Dynamics of Gelation in Polymerization Reactors Using the "Numerical Fractionation" Technique. Macromolecules, v. 27, n. 9, p. 2460-2469, 1994.

TOBITA, H.; HAMIELEC, A. E. Crosslinking kinetics in polyacrylamide networks. Polymer, v. 31, n. 8, p. 1546-1552, 1 ago. 1990.

TOBITA, H.; HAMIELEC, A. E. Kinetics of free-radical copolymerization: the pseudo-kinetic rate constant method. Polymer, v. 32, n. 14, p. 2641-2647, 1991.

TOBITA, H.; KUMAGAI, M.; AOYAGI, N. Microgel formation in emulsion polymerization. Polymer, v. 41, n. 2, p. 481-487, jan. 2000.

VERROS, G. D.; ACHILIAS, D. S.; GIANNOUKOS, G. I. Development of a comprehensive mathematical model for free radical suspension polymerization of methyl methacrylate. Polymer Engineering \& Science, v. 51, n. 4, p. 670-678, 1 abr. 2011.

WANG, G.; LI, M.; CHEN, X. Inverse suspension polymerization of sodium acrylate. Journal of Applied Polymer Science, v. 65, n. 4, p. 789-794, 1997. 


\section{APPENDICE}

APPENDICE A-. Chemical properties of reagents/Initial condition

\begin{tabular}{|c|c|c|c|c|}
\hline \multirow[b]{2}{*}{ Reagents } & \multicolumn{4}{|c|}{ Chemical Properties } \\
\hline & $\begin{array}{c}\text { Molar } \\
\text { Concentration } \\
\text { mol/L }\end{array}$ & $\begin{array}{c}\text { Boling } \\
\text { Temperature } \\
{ }^{\circ} \mathrm{C}\end{array}$ & $\begin{array}{c}\text { Melting } \\
\text { Temperature } \\
{ }^{\circ} \mathrm{C}\end{array}$ & $\begin{array}{c}\text { Density } \\
\mathrm{g} / \mathrm{ml}\end{array}$ \\
\hline Sodium Persulfate & 0.020207 & - & 180 & 2.59 \\
\hline Acrylic Acid & 2.0 & 141 & 14 & 1.05 \\
\hline Trimetylolpropane Triacrylate & 0.0004261 & 155 & - & 1.1 \\
\hline Tetraallytoxyethane & 0.00015012 & 160 & - & 1.06 \\
\hline Ethylene glycol dimethacrylate & 0.00017012 & 98 to $100^{\circ} \mathrm{C}$ & $-40^{\circ} \mathrm{C}$ & 1.051 \\
\hline
\end{tabular}

Ref: https://en.wikipedia.org/. 
APPENDICE B. Experimental runs-Matlab

\begin{tabular}{|l|ll|}
\hline Experiment & Time (min) & Conversion \\
\hline E1 & 0 & 0.043249056 \\
& 24 & 0.012490692 \\
& 34 & 0.088700536 \\
& 44 & 0.100011691 \\
& 54 & 0.102162219 \\
& 74 & 0.148058148 \\
& 84 & 0.463276812 \\
& 104 & 0.764417248 \\
& 114 & 0.993500687 \\
\hline
\end{tabular}

\begin{tabular}{|l|ll|}
\hline Experiment & Time (min) & \multicolumn{1}{c|}{ Conversion } \\
\hline E2 & 0 & 0.251331285 \\
& 24 & 0.26842 \\
& 34 & 0.27823918 \\
& 44 & 0.369273455 \\
& 54 & 0.405575175 \\
& 64 & 0.48117 \\
& 84 & 0.880286592 \\
\hline
\end{tabular}

\begin{tabular}{|l|ll|}
\hline Experiment & Time (min) & \multicolumn{1}{c|}{ Conversion } \\
\hline E3 & 0 & 0.097795456 \\
& 24 & 0.126431666 \\
& 34 & 0.091220091 \\
& 44 & 0.12666868 \\
& 64 & 0.114684696 \\
& 74 & 0.148845114 \\
& 94 & 0.118550449 \\
& 124 & 0.07202903 \\
& 144 & 0.07182901 \\
& 164 & 0.086728234 \\
& 184 & 0.124342619 \\
& 200 & 0.284232218 \\
& 215 & 0.44624250 \\
& 230 & 0.622375982 \\
& 245 & 0.684803436 \\
\hline
\end{tabular}




\begin{tabular}{|l|ll|}
\hline Experiment & Time (min) & \multicolumn{1}{c|}{ Conversion } \\
\hline E4 & 0 & 0.097493802 \\
& 21 & 0.105973479 \\
& 35 & 0.120582229 \\
& 40 & 0.138144297 \\
& 50 & 0.47028463 \\
& 60 & 0.166950143 \\
& 70 & 0.178701159 \\
& 90 & 0.186634972 \\
& 100 & 0.238174457 \\
& 110 & 0.387575014 \\
& 120 & 0.5828151 \\
& 130 & 0.608618306 \\
& 150 & 0.634664957 \\
& 160 & 0.708665713 \\
& 180 & 0.809112596 \\
\hline
\end{tabular}

\begin{tabular}{|c|c|c|c|}
\hline Experiment & Time (min) & Conversion & Weigth Fraction of Gel \\
\hline \multirow[t]{12}{*}{ E5 } & 0 & 0.110130208 & 0.0 \\
\hline & 10 & 0.135663663 & - \\
\hline & 25 & 0.161405107 & 0.205973899 \\
\hline & 33 & 0.226636829 & 0.250360284 \\
\hline & 40 & 0.3549 & 0.289336882 \\
\hline & 50 & 0.3590 & 0.34805333 \\
\hline & 60 & 0.35958 & 0.362254227 \\
\hline & 70 & 0.366598 & 0.375495551 \\
\hline & 85 & 0.3697768 & 0.395426565 \\
\hline & 95 & 0.431224878 & 0.397014742 \\
\hline & 140 & 0.565239022 & 0.495266857 \\
\hline & 160 & 1.00 & 0.507159638 \\
\hline
\end{tabular}

\begin{tabular}{|l|ll|}
\hline Experiment & Time (min) & \multicolumn{1}{c|}{ Conversion } \\
\hline E6 & 0 & 0.011133000 \\
& 24 & 0.027474496 \\
& 34 & 0.097241773 \\
& 44 & 0.107922156 \\
& 54 & 0.0 \\
& 64 & 0.0 \\
& 84 & 0.342671036 \\
& 104 & 0.433780664 \\
& 114 & 0.634277049 \\
\hline
\end{tabular}




\begin{tabular}{|l|ll|}
\hline Experiment & Time (min) & \multicolumn{1}{c|}{ Conversion } \\
\hline E7 & 0 & 0.063975567 \\
& 24 & 0.064305333 \\
& 34 & 0.076461577 \\
& 44 & 0.095381511 \\
& 54 & 0.24296089 \\
& 64 & 0.341628887 \\
& 84 & 0.4230016 \\
& 104 & 0.463378045 \\
& 114 & 0.547474938 \\
& 124 & 0.57902614 \\
\hline
\end{tabular}

\begin{tabular}{|l|ll|}
\hline Experiment & Time (min) & \multicolumn{1}{c|}{ Conversion } \\
\hline E8 & 0 & 0.075541038 \\
& 24 & 0.1842 \\
& 34 & 0.1864 \\
& 44 & 0.2024 \\
& 64 & 0.2102 \\
& 74 & 0.2329 \\
& 94 & 0.3008 \\
& 124 & 0.5182 \\
& 144 & 0.5613 \\
& 164 & 0.5715 \\
& 184 & 0.6097 \\
& 200 & 0.7215 \\
& 215 & 0.7229 \\
& 230 & 0.7318 \\
& 245 & 0.7920 \\
\hline
\end{tabular}




\begin{tabular}{|l|ll|}
\hline Experiment & Time (min) & \multicolumn{1}{c|}{ Conversion } \\
\hline E9 & 0 & 0.0079266 \\
& 24 & 0.012491 \\
& 34 & 0.029973 \\
& 44 & 0.033147 \\
& 64 & 0.057132 \\
& 74 & 0.06973 \\
& 94 & 0.088701 \\
& 124 & 0.10001 \\
& 144 & 0.10216 \\
& 164 & 0.14806 \\
& 184 & 0.3481 \\
& 200 & 0.46328 \\
& 215 & 0.643 \\
& 230 & 0.76442 \\
& 245 & 0.80349 \\
\hline
\end{tabular}

\begin{tabular}{|l|lll|}
\hline $\begin{array}{l}\text { Experiment } \\
\text { S }\end{array}$ & Time $(\mathrm{min})$ & & Weigth Fraction of Gel \\
\hline E10 & 0 & 0.029586 & 0.0 \\
& 10 & 0.0842579 & 0.15421458 \\
& 25 & 0.186918 & 0.239066402 \\
& 33 & 0.1889047 & 0.283167632 \\
& 40 & 0.4311739 & 0.334243273 \\
& 50 & 0.4473012 & 0.376115165 \\
& 60 & 0.5225997 & 0.408828547 \\
& 70 & 0.676308 & 0.462585507 \\
& 85 & 0.6877301 & 0.512871323 \\
& 95 & 0.7147112 & 0.541705286 \\
& 140 & 0.9177995 & 0.609218052 \\
\hline
\end{tabular}




\begin{tabular}{|l|ll|}
\hline Experiment & Time $(\mathrm{min})$ & \\
\hline E11 & 0 & 0.1479 \\
& 10 & 0.1499 \\
& 20 & 0.2369 \\
& 30 & 0.3696 \\
& 40 & 0.3960 \\
& 50 & 0.4560 \\
& 60 & 0.4857 \\
& 70 & 0.5116 \\
& 80 & 0.5177 \\
& 90 & 0.5234 \\
& 100 & 0.5793 \\
& 110 & 0.5994 \\
& 120 & 0.6835 \\
& 135 & 0.6841 \\
& 145 & 0.6874 \\
& 150 & 0.7095 \\
& 170 & 0.7980 \\
& 180 & 0.8139 \\
& 200 & 0.8268 \\
& 220 & 0.9827 \\
\hline
\end{tabular}

\begin{tabular}{|l|ll|}
\hline Experiment & Time (min) & \\
& & Conversion \\
\hline E12 & 0 & 0.01432 \\
& 30 & 0.04389 \\
& 45 & 0.07369 \\
& 55 & 0.1295 \\
& 65 & 0.2332 \\
& 75 & 0.26417 \\
& 85 & 0.28303 \\
& 95 & 0.33943 \\
& 100 & 0.40235 \\
& 105 & 0.41775 \\
& 115 & 0.43712 \\
& 120 & 0.45419 \\
& 135 & 0.54149 \\
& 145 & 0.69996 \\
& & \\
& 155 & 0.75782 \\
\hline
\end{tabular}




\begin{tabular}{|l|ll|}
\hline Experiment & Time (min) & \\
& & Conversion \\
\hline E13 & 0 & 0.0 \\
& 6 & 0.1311 \\
& 15 & 0.4445 \\
& 30 & 0.8542 \\
& 60 & 0.9717 \\
& 90 & 1.0000 \\
\hline
\end{tabular}

\begin{tabular}{|l|lll|}
\hline Experiment & Time (min) & Weigth Fraction of Gel \\
\multicolumn{1}{|l}{ E14 } & 0 & 0.158208 & 0.0 \\
& 10 & 0.2303 & 0.070206638 \\
& 25 & 0.283046 & 0.151367306 \\
& 33 & 0.291613 & 0.213953488 \\
& 40 & 0.313634 & 0.0 \\
& 50 & 0.393499 & 0.0 \\
& 60 & 0.494708 & 0.26011037 \\
& 70 & 0.666914 & 0.408828547 \\
& 85 & 0.918488 & 0.516111248 \\
& 95 & 0.972407 & 0.633534707 \\
\hline
\end{tabular}

\begin{tabular}{|l|ll|}
\hline Experiment & Time (min) & Conversion \\
\hline E15 & 0 & 0.044885116 \\
& 24 & 0.049151795 \\
& 34 & 0.195541726 \\
& 44 & 0.252565117 \\
& 54 & 0.314998955 \\
& 64 & 0.376194381 \\
& 84 & 0.536481144 \\
& 104 & 0.753522849 \\
& 114 & 0.776937493 \\
& 124 & 0.782085712 \\
& 144 & 0.88997721 \\
\hline
\end{tabular}




\begin{tabular}{|l|ll|}
\hline Experiment & Time (min) & \multicolumn{1}{c|}{ Conversion } \\
\hline E16 & 0 & 0.11208225 \\
& 24 & 0.23545104 \\
& 34 & 0.27415721 \\
& 44 & 0.54802538 \\
& 64 & 0.55974058 \\
& 74 & 0.74926421 \\
& 94 & 0.88681986 \\
& 124 & 0.9381462 \\
& 144 & 0.96539589 \\
& 164 & 0.99786944 \\
\hline
\end{tabular}

\begin{tabular}{|l|ll|}
\hline Experiment & Time (min) & \multicolumn{1}{c|}{ Conversion } \\
\hline E17 & 0 & 0.0717 \\
& 24 & 0.08501 \\
& 34 & 0.11924 \\
& 44 & 0.29743 \\
& 54 & 0.33762 \\
& 64 & 0.69662 \\
& 104 & 0.77036 \\
& 134 & 0.7874 \\
& 154 & 0.17967 \\
& 174 & 0.83599 \\
\hline
\end{tabular}

\begin{tabular}{|l|ll|}
\hline Experiment & Time (min) & \multicolumn{1}{c|}{ Conversion } \\
\hline E18 & 0 & 0.12922563 \\
& 24 & 0.1358834 \\
& 34 & 0.16865779 \\
& 44 & 0.1778416 \\
& 54 & 0.22123919 \\
& 64 & 0.3552454 \\
& 104 & 0.57347872 \\
& 134 & 0.73470495 \\
& 154 & 0.759961 \\
& 174 & 0.77686238 \\
\hline
\end{tabular}




\section{APPENDICE C- MATLAB CODE}

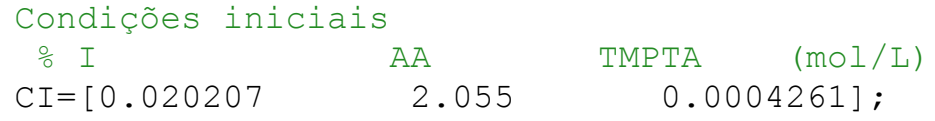

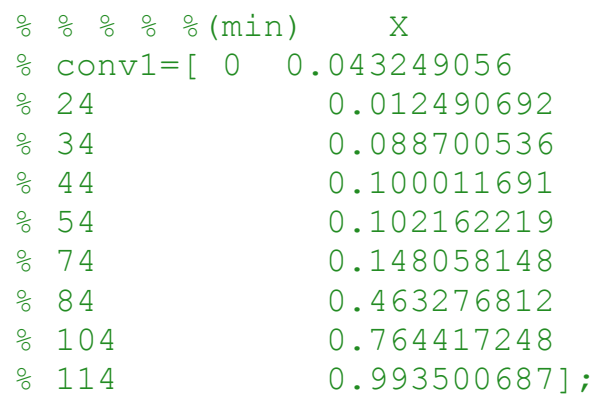

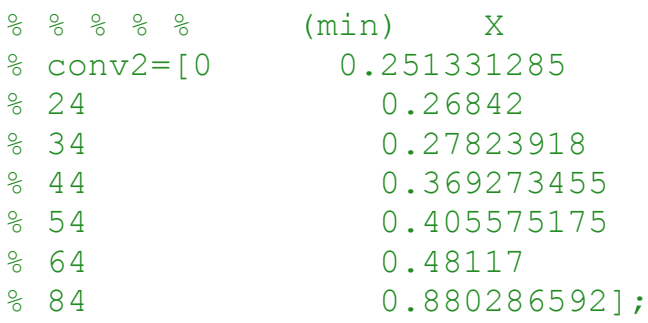




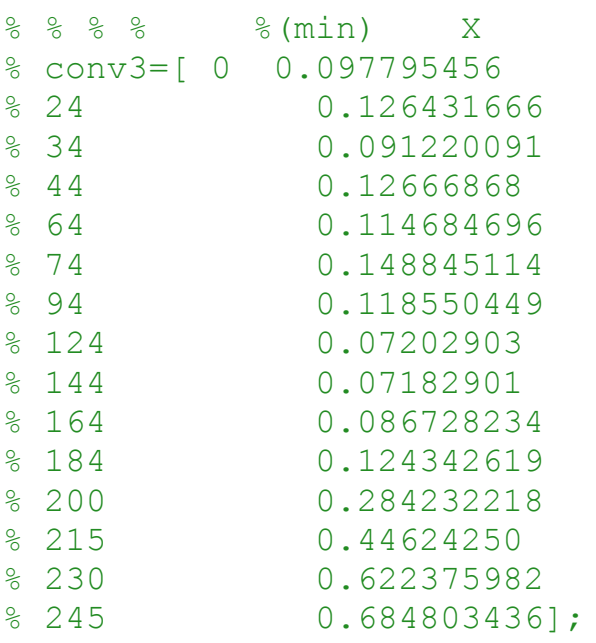

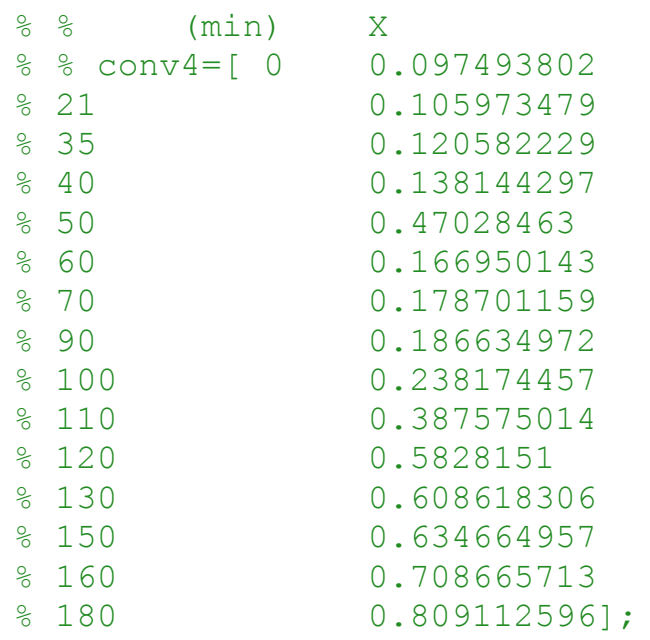

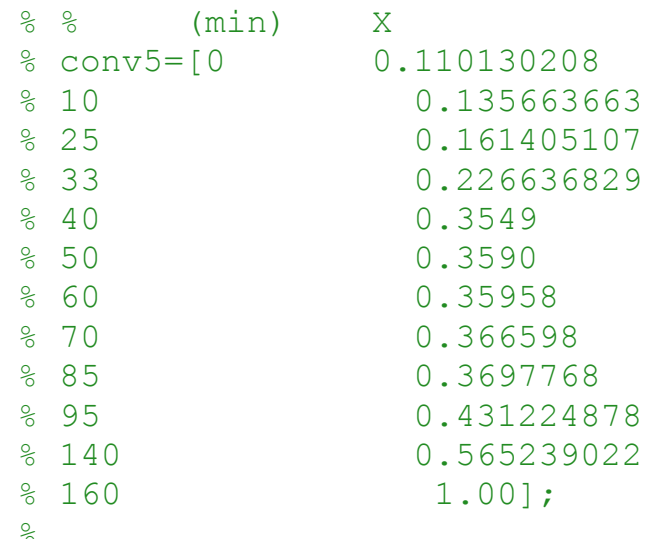




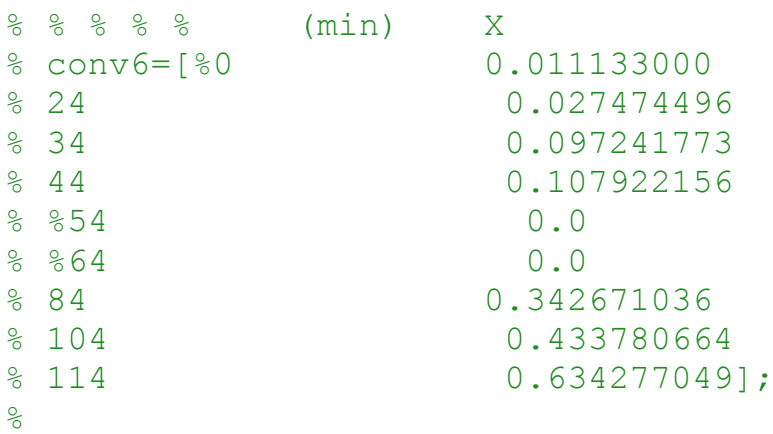

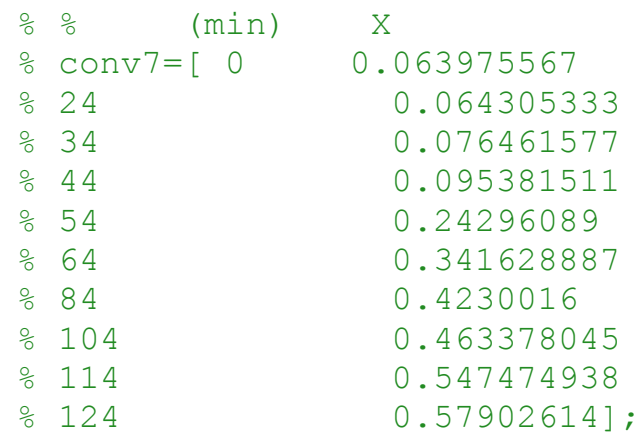

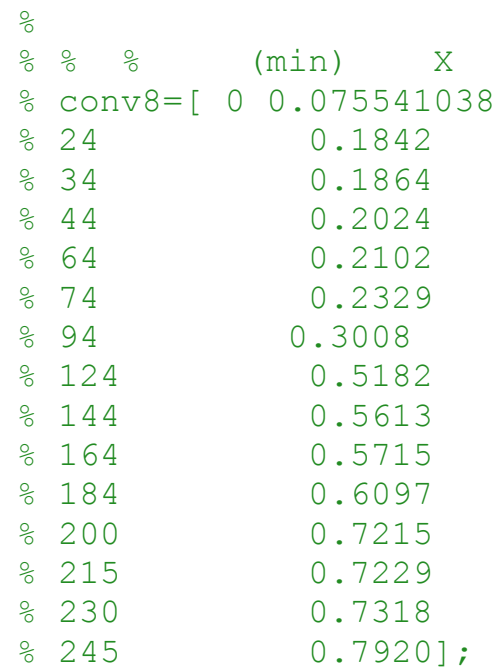




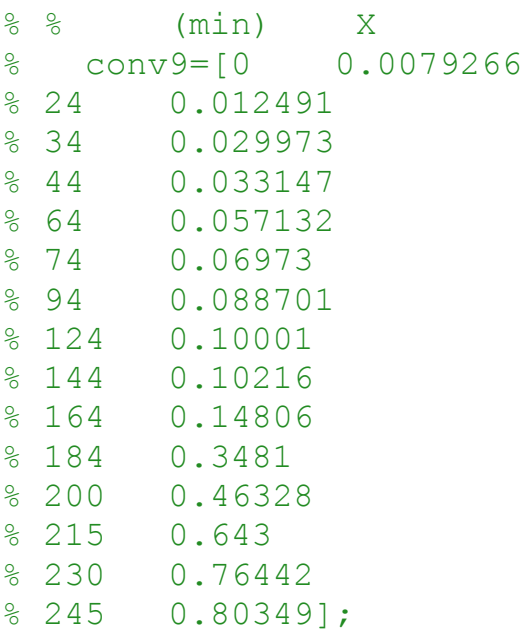

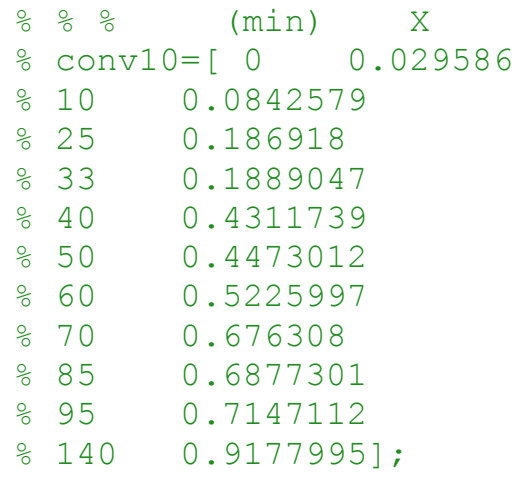

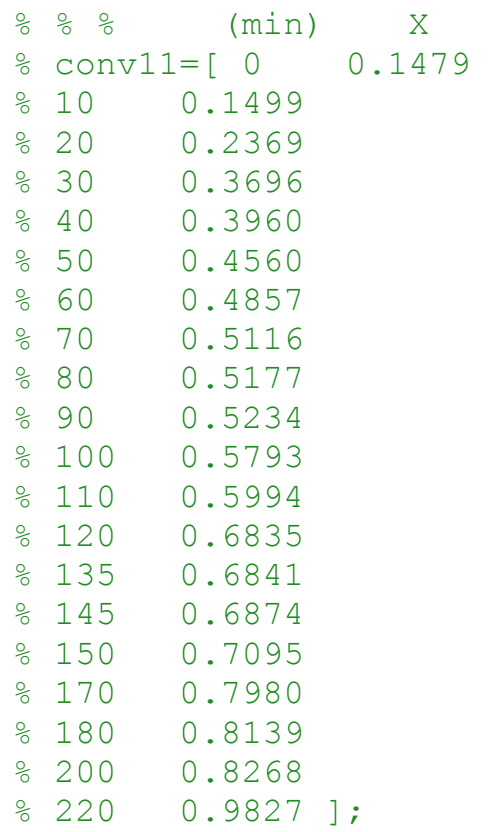


\%

$\div \quad \div(\min ) \quad \mathrm{X}$

conv12=[ $\begin{array}{ll}0 & 0.01432\end{array}$

$\begin{array}{ll}\circ 30 & 0.04389\end{array}$

4 0.07369

이인 0.1295

은 650.2332

$\circ 75 \quad 0.26417$

\% $85 \quad 0.28303$

잉 0.33943

\% $100 \quad 0.40235$

잉 0.41775

\% 1150.43712

잉 0.45419

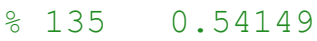

$\begin{array}{lll}\circ & 145 & 0.69996\end{array}$

응 1550.75782$]$;

\begin{tabular}{|c|c|c|c|}
\hline$\%$ & $(\min )$ & $\mathrm{X}$ & \\
\hline$\frac{0}{0}$ & conv13=[ 0 & 0 & 0.0 \\
\hline \% & 6 & & 0.1311 \\
\hline 응 & 15 & & 0.4445 \\
\hline$\%$ & 30 & & 0.8542 \\
\hline$\%$ & 60 & & 0.9717 \\
\hline & 90 & & $1.0000] ;$ \\
\hline
\end{tabular}

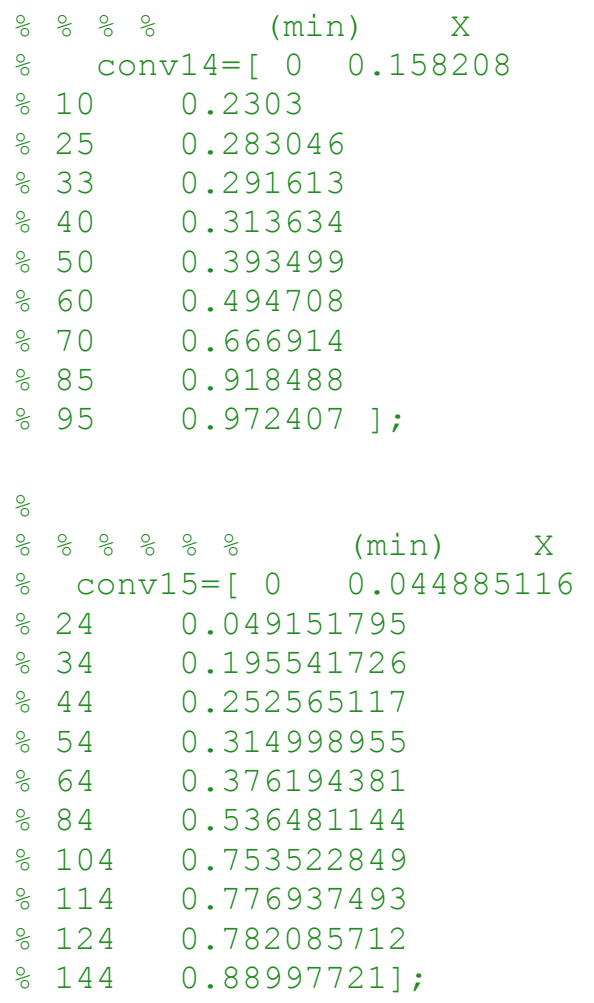




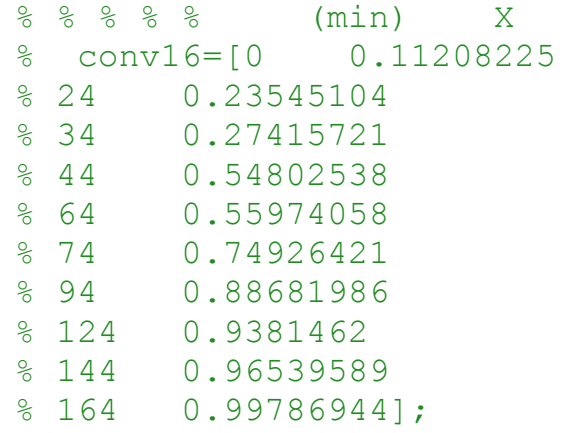

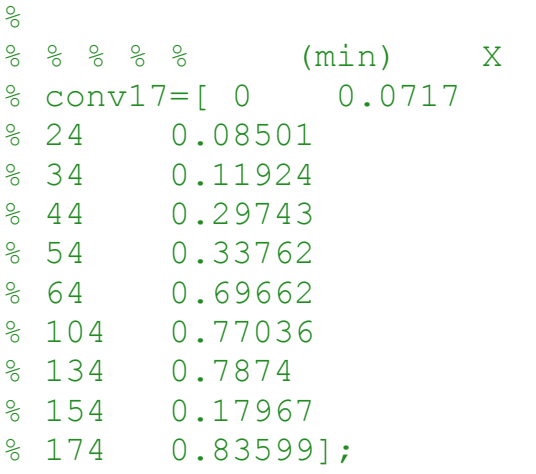

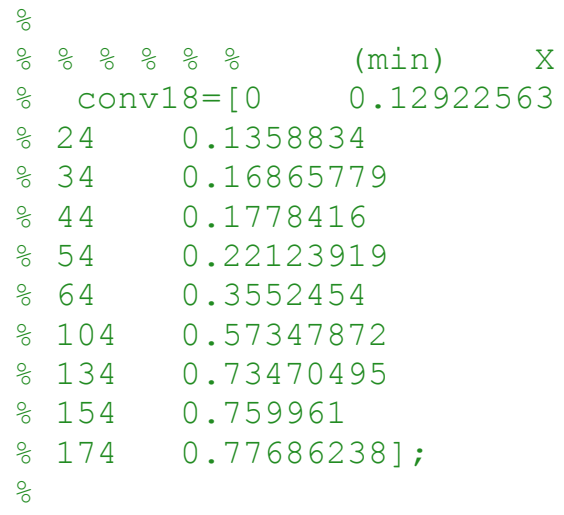


\% Fração de gel

\% $\frac{\circ}{0} \% \quad t \quad(\min ) \quad \mathrm{Wg}$

$\begin{array}{ll}\text { fge } 1=[0 & 0.0 \\ 10 & 0.15421458 \\ 25 & 0.239066402 \\ 33 & 0.283167632 \\ 40 & 0.334243273 \\ 50 & 0.376115165 \\ 60 & 0.408828547 \\ 70 & 0.462585507 \\ 85 & 0.512871323 \\ 95 & 0.541705286 \\ 120 & 0.609218052] ;\end{array}$

$\circ \frac{\circ}{0} \quad t(\min ) \quad \mathrm{Wg}$

\% fgel2 = [ $\begin{array}{lll}0 & 0 & 0\end{array}$

잉 250.205973899

이잉 0.250360284

\% $40 \quad 0.289336882$

$50 \quad 0.34805333$

\% $60 \quad 0.362254227$

\% $70 \quad 0.375495551$

응 0.395426565

\%95 0.397014742

\% $120 \quad 0.495266857$

이잉 0.507159638 ]

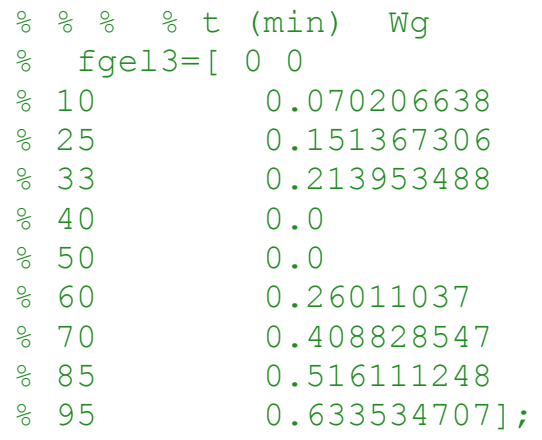

\% conv vs fgel

\% convgel1= [

$\begin{array}{lll}\circ & 0.029586 & 0.0\end{array}$

\% $0.0842579 \quad 0.1542146$

\% $0.186918 \quad 0.2390664$

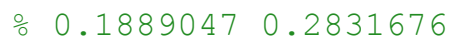

\% 0.43117390 .3342433

$\begin{array}{lll}\circ & 0.4473012 & 0.3761152\end{array}$

\% 0.46979130 .4088285 


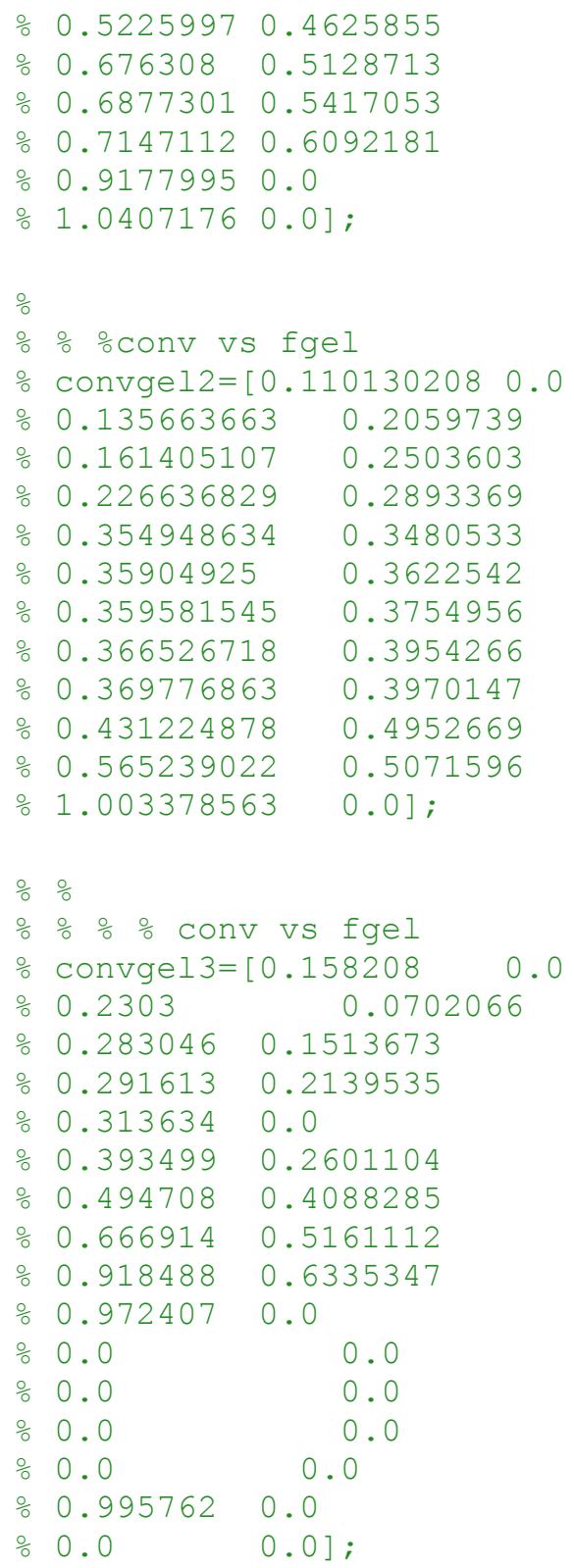

\% Parâmetros cinéticos

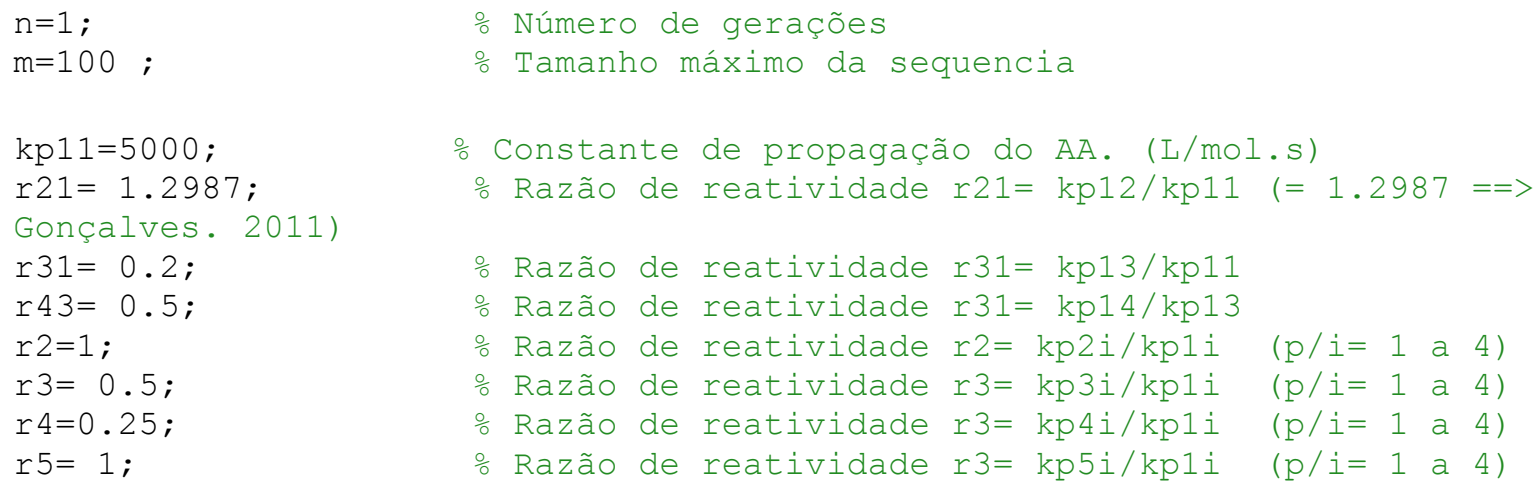



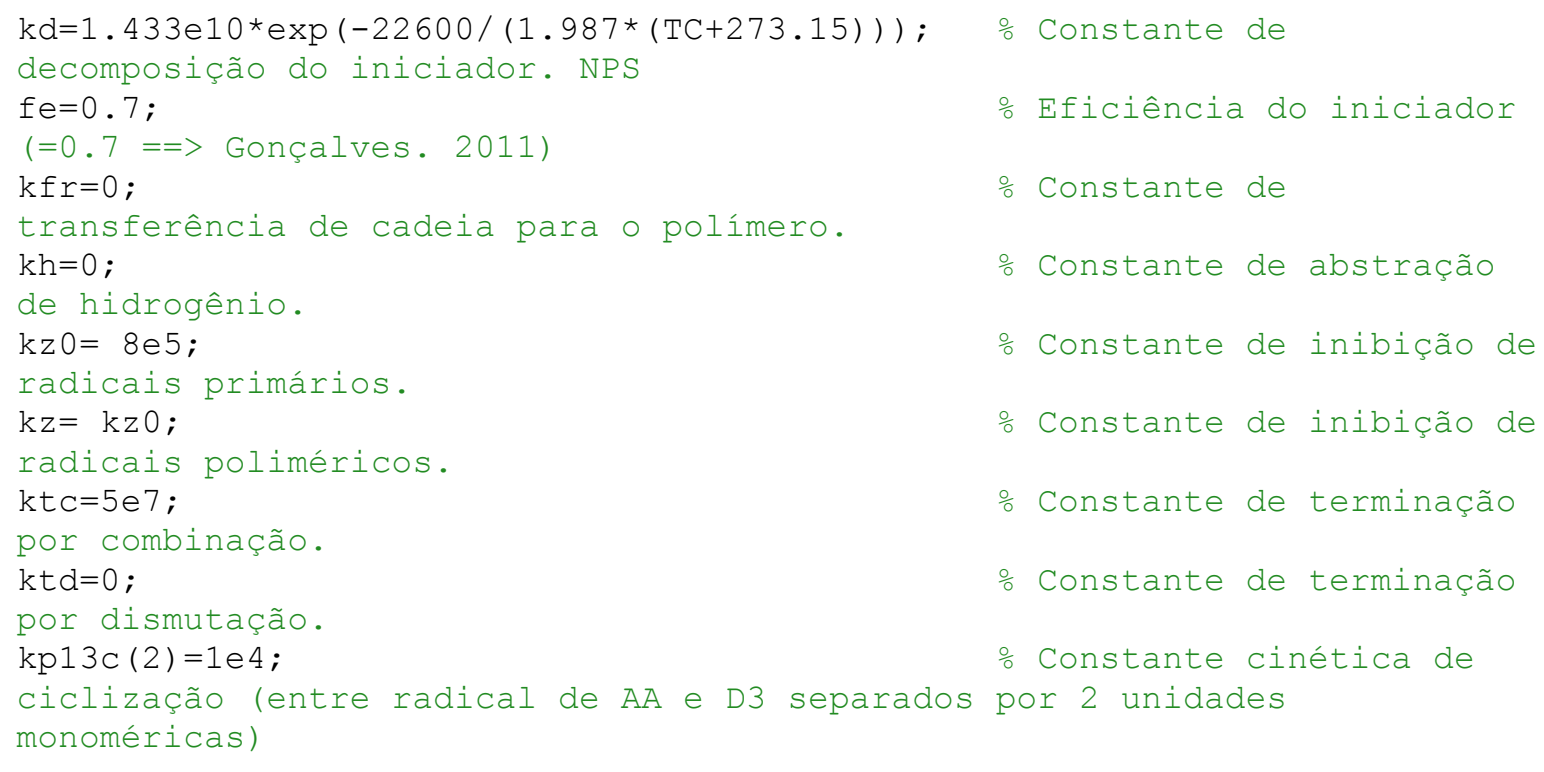


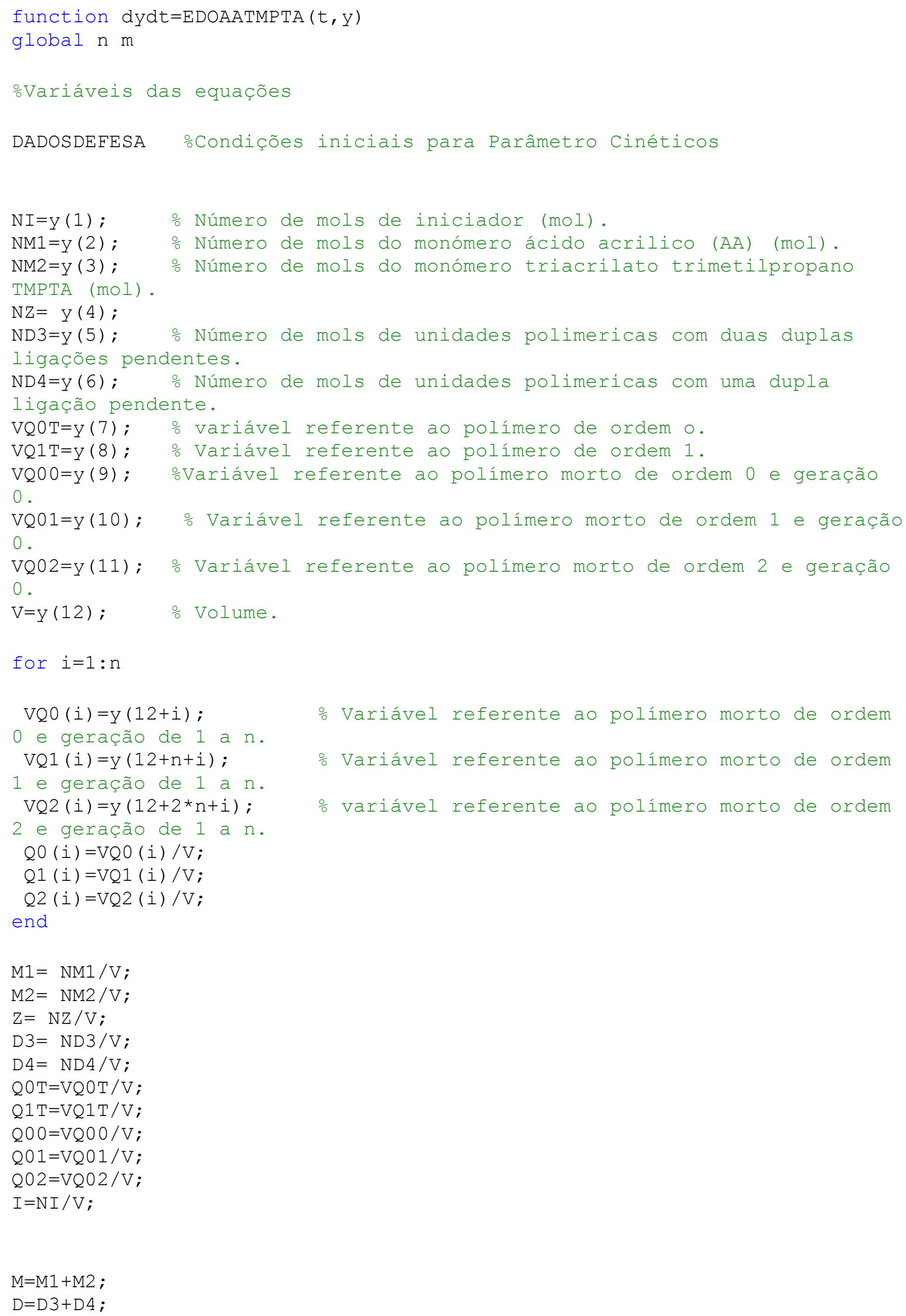




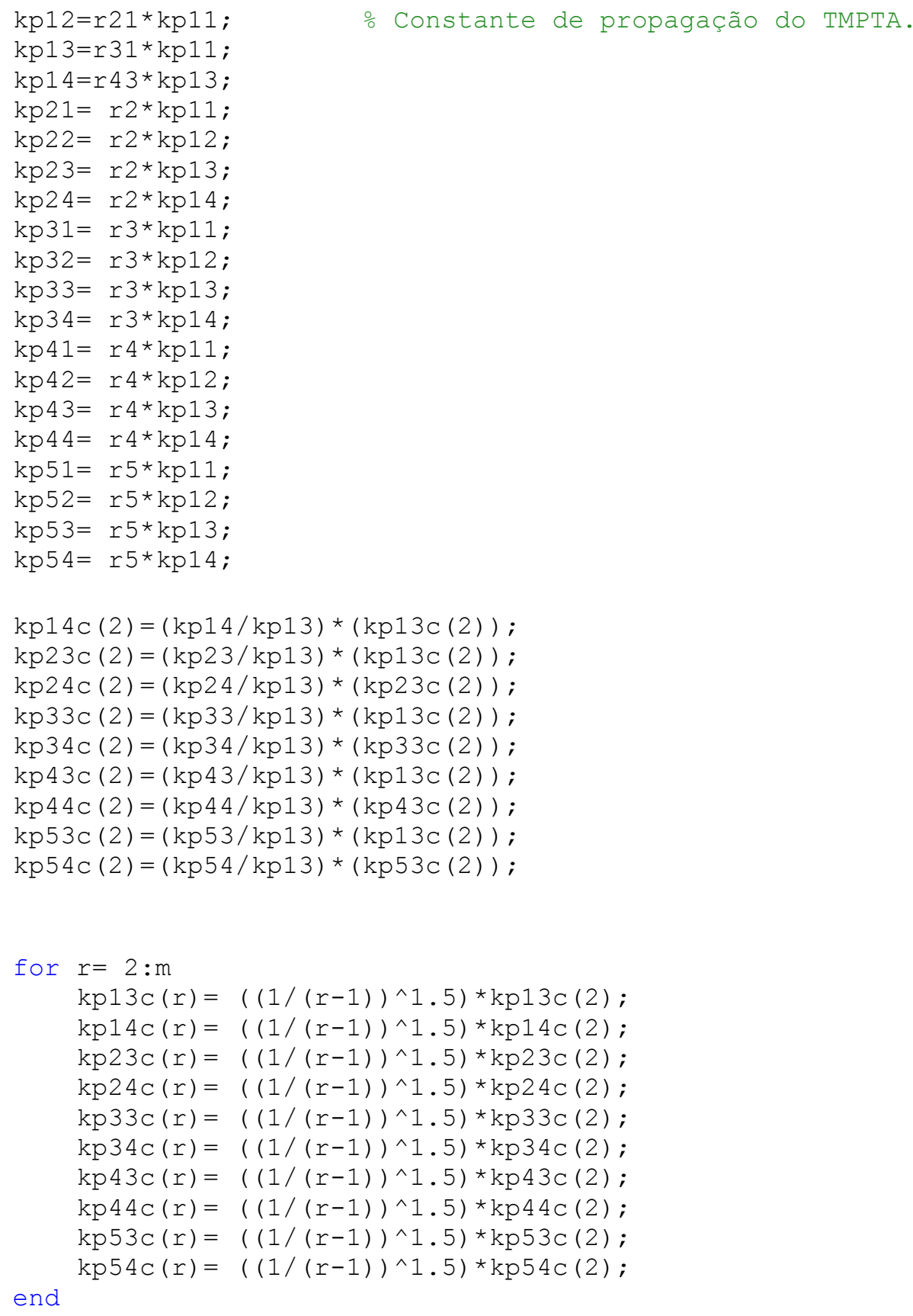




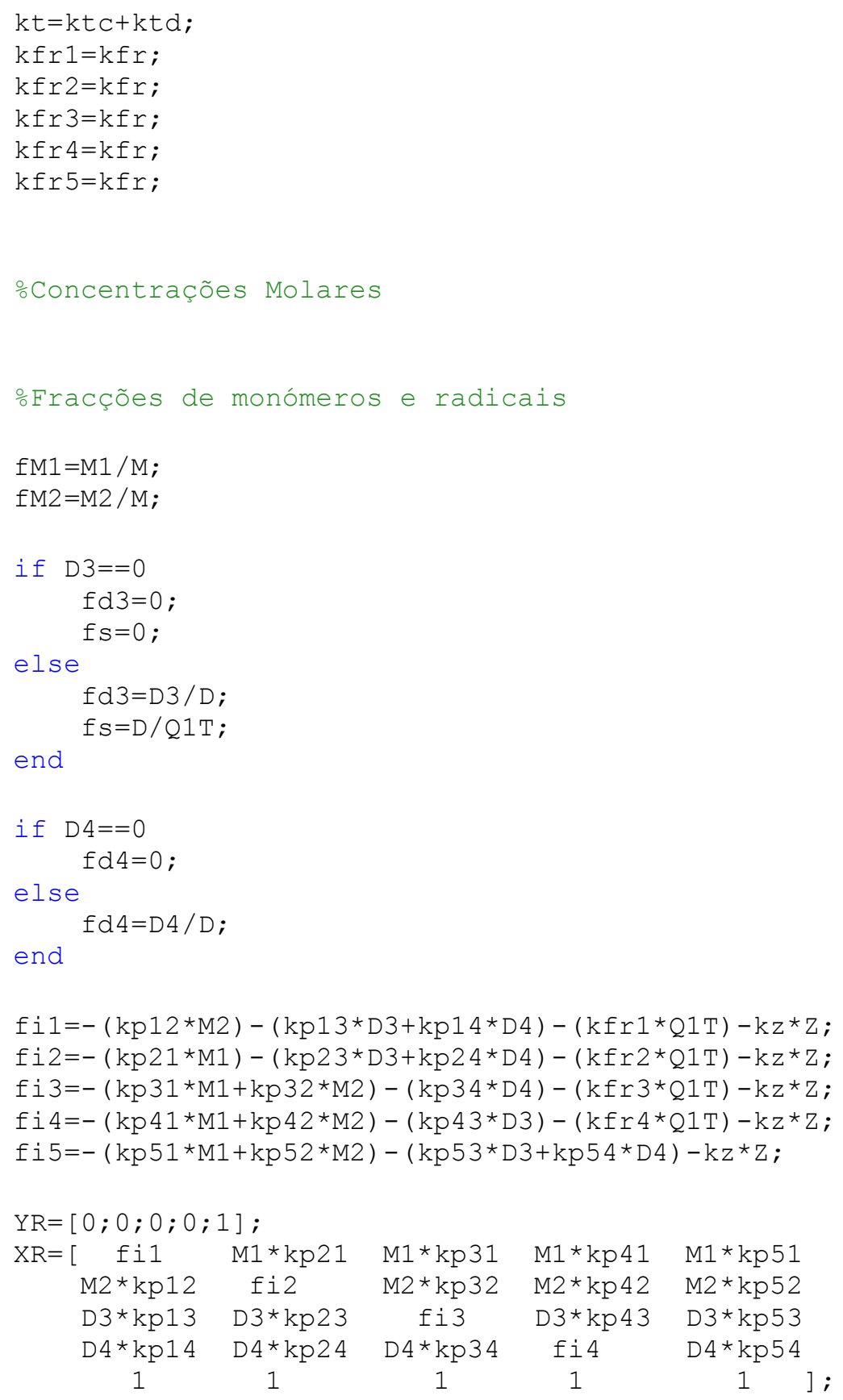




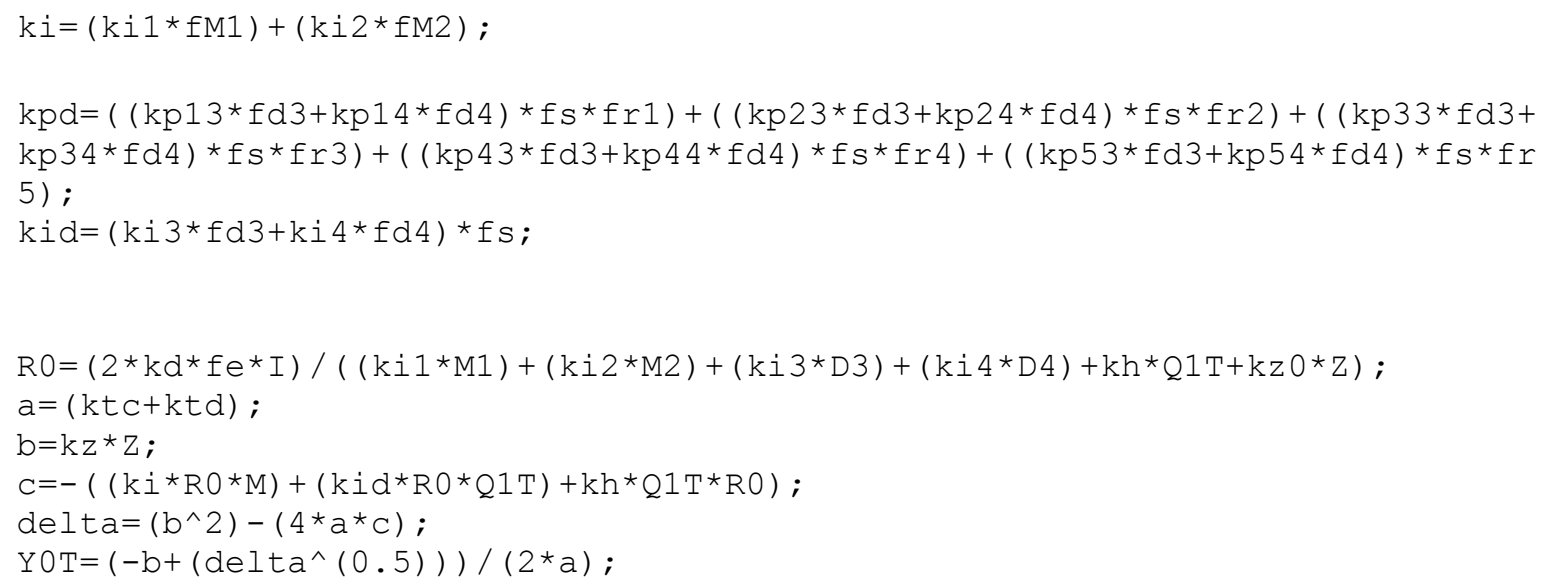




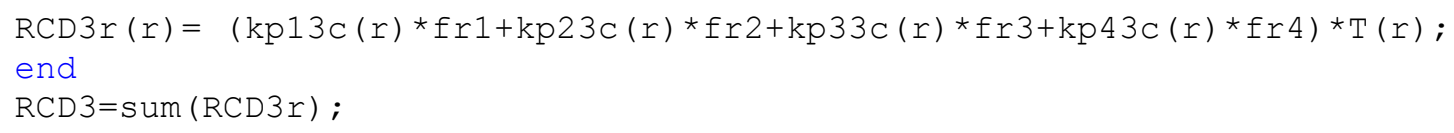




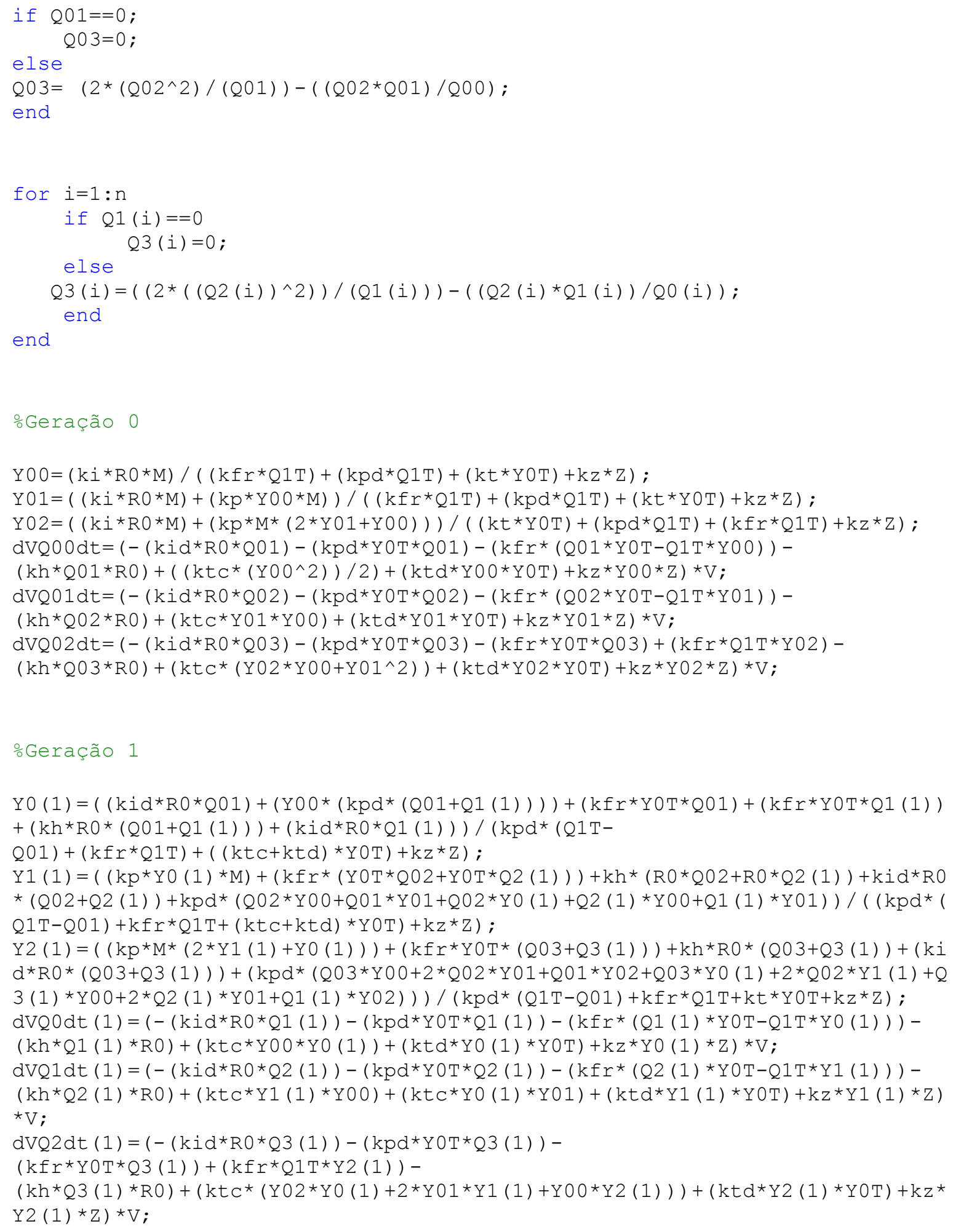




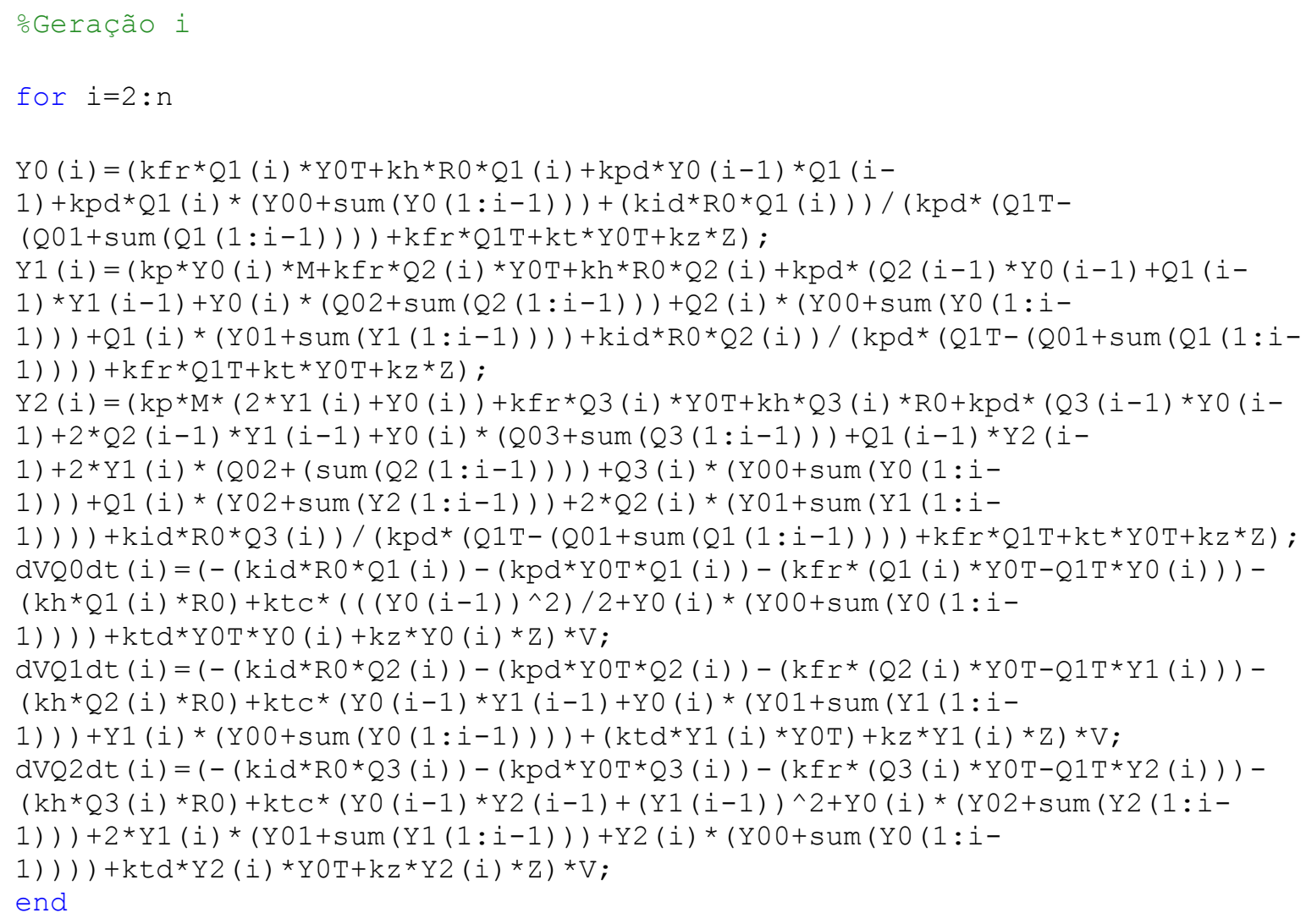




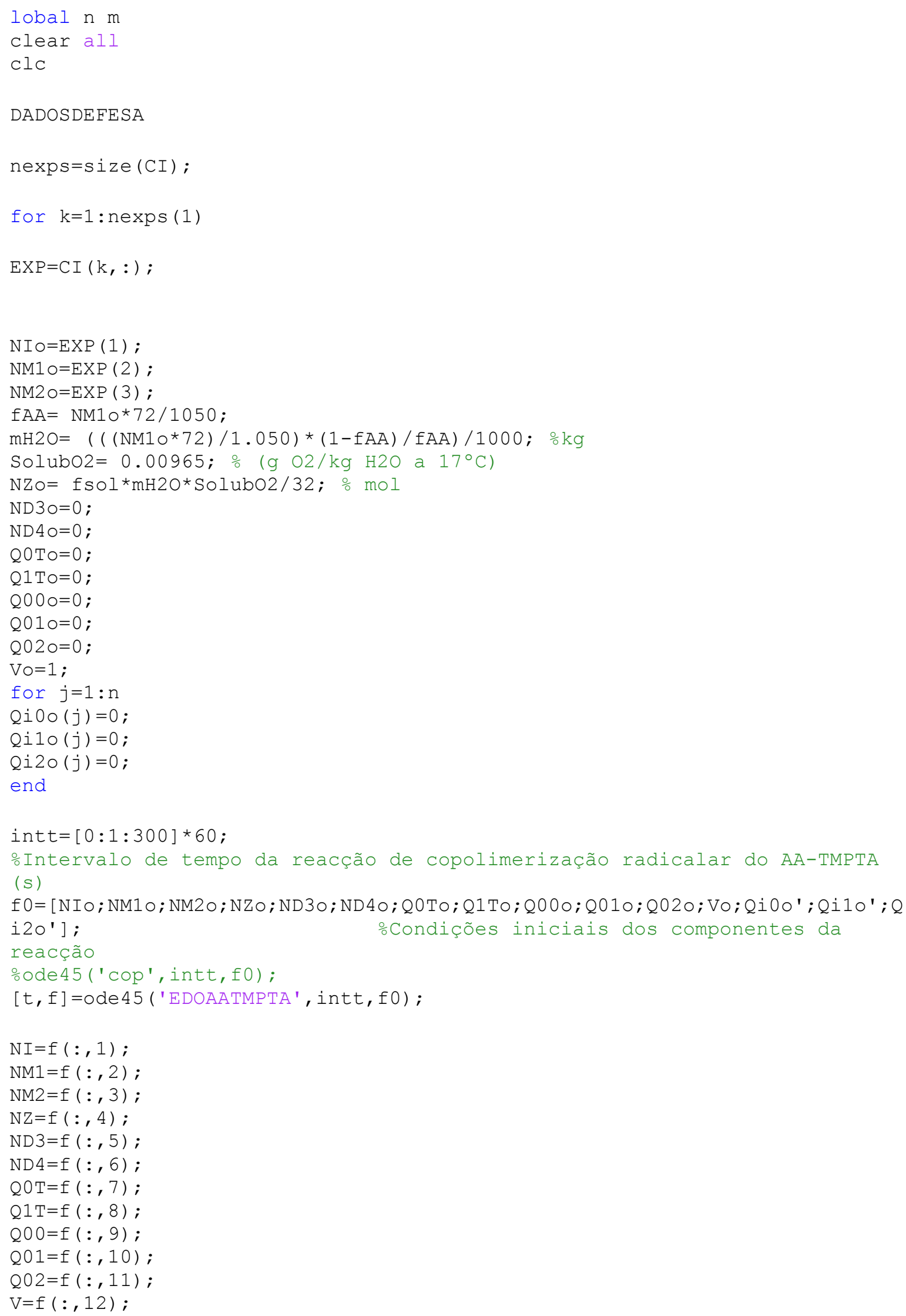




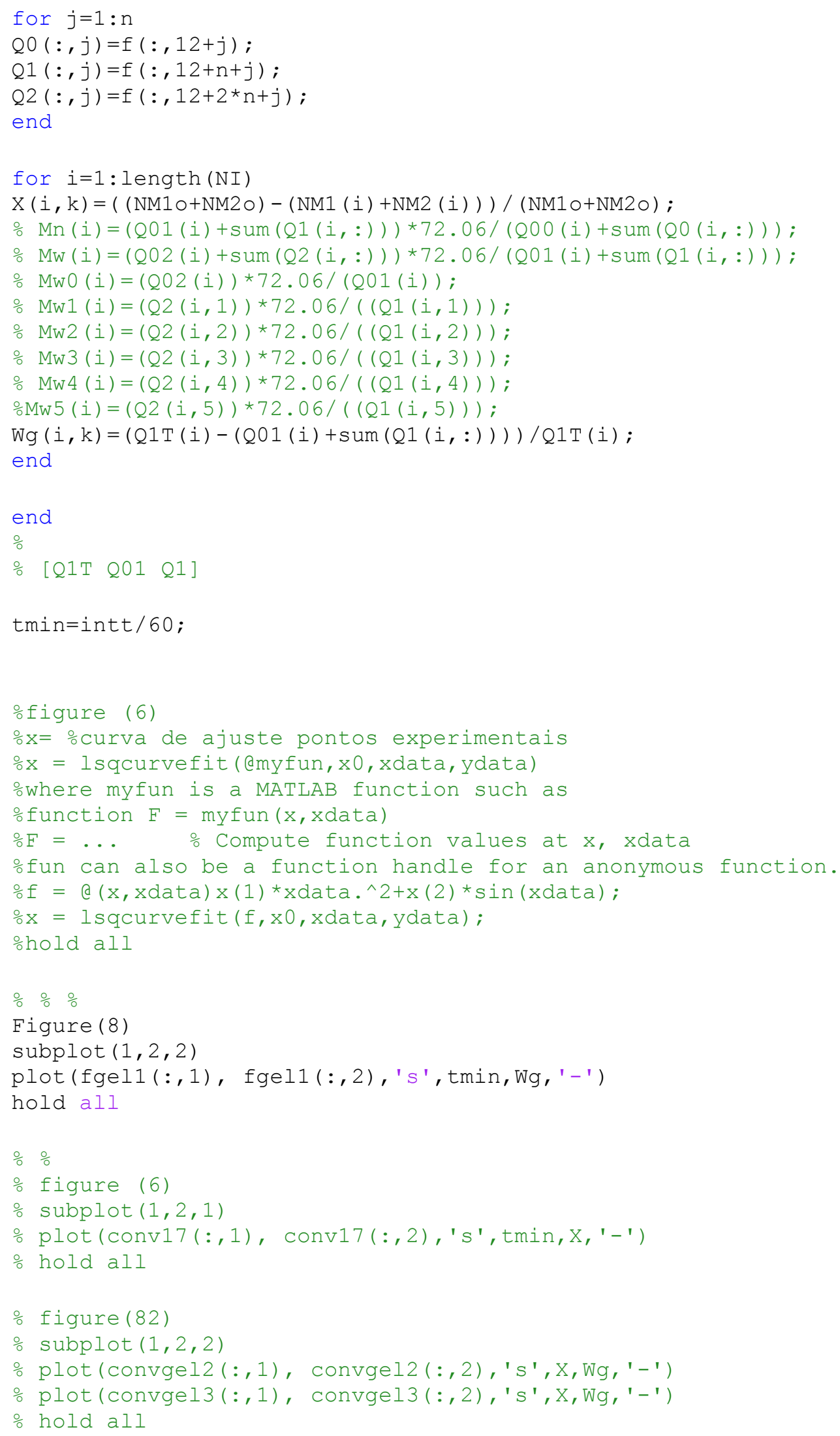

